\title{
WestVirginiaUniversity
}

THE RESEARCH REPOSITORY @ WVU

Graduate Theses, Dissertations, and Problem Reports

2005

\section{Reconstruction of fingerprints from minutiae points}

Jidnya A. Shah

West Virginia University

Follow this and additional works at: https://researchrepository.wvu.edu/etd

\section{Recommended Citation}

Shah, Jidnya A., "Reconstruction of fingerprints from minutiae points" (2005). Graduate Theses, Dissertations, and Problem Reports. 1683.

https://researchrepository.wvu.edu/etd/1683

This Thesis is protected by copyright and/or related rights. It has been brought to you by the The Research Repository @ WVU with permission from the rights-holder(s). You are free to use this Thesis in any way that is permitted by the copyright and related rights legislation that applies to your use. For other uses you must obtain permission from the rights-holder(s) directly, unless additional rights are indicated by a Creative Commons license in the record and/ or on the work itself. This Thesis has been accepted for inclusion in WVU Graduate Theses, Dissertations, and Problem Reports collection by an authorized administrator of The Research Repository @ WVU. For more information, please contact researchrepository@mail.wvu.edu. 


\title{
Reconstruction of Fingerprints from Minutiae Points
}

\author{
by \\ Jidnya A. Shah \\ Thesis submitted to the \\ College of Engineering and Mineral Resources \\ at West Virginia University \\ in partial fulfillment of the requirements \\ for the degree of
}

Master of Science

in

Electrical Engineering

Arun A. Ross, Ph.D., Chair

Xin Li, Ph.D.

Larry Hornak, Ph.D.

Lane Department of Computer Science and Electrical Engineering

Morgantown, West Virginia

2005

Keywords: Minutiae template, Fingerprint individuality, Classification, Reconstruction, Gabor filters, Streamlines, Line Integral Convolution.

Copyright 2005 Jidnya A. Shah 


\author{
Abstract \\ Reconstruction of Fingerprints from Minutiae Points \\ by \\ Jidnya A. Shah \\ Master of Science in Electrical Engineering \\ West Virginia University \\ Arun A. Ross, Ph.D., Chair
}

Most fingerprint authentication systems utilize minutiae information to compare fingerprint images. During enrollment, the minutiae template of a user's fingerprint is extracted and stored in the database. In this work, we concern ourselves with the amount of fingerprint information that can be elicited from the minutiae template of a user's fingerprint. We demonstrate that minutiae information can reveal substantial details such as the orientation field and class of the (unseen) parent fingerprint that can potentially be used to reconstruct the original fingerprint image.

Given a minutiae template, the proposed method first estimates the orientation map of the parent fingerprint by constructing minutiae triplets. The estimated orientation map is observed to be remarkably consistent with the underlying ridge flow of the unseen parent fingerprint. We also discuss a fingerprint classification technique that utilizes only the minutiae information to determine the class of the fingerprint (Arch, Left loop, Right loop and Whorl). The proposed classifier utilizes various properties of the minutiae distribution such as angular histograms, density, relationship between minutiae pairs, etc. A classification accuracy of $82 \%$ is obtained on a subset of the NIST-4 database. This indicates that the seemingly random minutiae distribution of a fingerprint can reveal important class information.

Furthermore, contrary to what has been claimed by several minutiae-based fingerprint system vendors, we demonstrate that the minutiae template of a user may be used to reconstruct fingerprint images. Two techniques have been proposed for fingerprint reconstruction. The first technique utilizes Gabor-like filters, while the second technique employs streamlines and Linear Integral Convolution (LIC) to generate the ridge structure of the parent fingerprint. The salient feature of the second method is its ability to generate minutiae at desired locations in the regenerated ridge map. Experiments conducted on the minutiae templates of the NIST-4 database challenge the commonly held notion that minutiae points do not reveal information about the parent fingerprint. 
I dedicate my thesis to my family 


\section{Acknowledgments}

Words alone cannot fully express my gratitude and appreciation for those who guided and supported me through these last two years. I have been fortunate to be surrounded by excellent teachers and friends.

It was my honor to have Dr. Arun Ross as my advisor and committee chair. His complete dedication to work, love for perfection, infectious enthusiasm, have always motivated me. My interactions with him have been of immense help in defining my research goals and in finding the systematic solutions to achieve them. His regular reminders of "work hard" often pushed me to put in my best possible efforts.

I am very grateful to Dr. Xin Li and Dr. Lawrence Hornak for their valuable guidance and suggestions for my thesis. Their comments have been very useful in enhancing the presentation of this thesis. I would also like to extend my gratitude to all lab-mates Rohin, Sarvesh, Simona, Pisut, Chris, Rohan and Phani who have been with me all the time and for their co-operation and necessary feedback. I am also very thankful to Kiran for helping me to use the SDK's of various commercial fingerprint system products. My heartfelt thanks to the department secretaries and the systems group for their excellent work and support.

I would like to thank my parents, sister, brother and my in laws for their support, neverfading love and sacrifice. It was because of them I could survive hard times and be consistent on my work. A big thanks to my husband, Samir, for understanding me, standing by me and sharing my joys and frustrations without which I would not have come this far. 


\section{Contents}

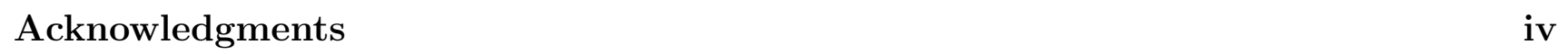

List of Figures $\quad$ vi

List of Tables $\quad$ ix

1 Introduction 1

1.1 Biometrics . . . . . . . . . . . . . . . . . . . . . 1

1.2 Fingerprints . . . . . . . . . . . . . . . . . . . . 4

1.3 Vulnerabilities of a biometric system . . . . . . . . . . . . . . . 6

1.3 .1 Vulnerability of a biometric template . . . . . . . . . . . . . . . . 8

1.4 Problem Statement . . . . . . . . . . . . . . . . . . . . . . . . . . . . . . . . . . . . . . . . . .

1.5 Contribution of the Thesis . . . . . . . . . . . . . . . . . . . 13

1.6 Organization of the Thesis $\ldots \ldots \ldots \ldots \ldots$

2 Estimating Ridge Orientations using Minutiae Points 15

2.1 Orientation Estimation Algorithm . . . . . . . . . . . . . . . . . . . . 16

2.2 Validating the estimated orientation map . . . . . . . . . . . . . . . . . . . 22

3 Fingerprint Classification Using Minutiae Points $\quad 27$

3.1 Fingerprint classification . . . . . . . . . . . . . . . . . . . 27

3.1 .1 Need for fingerprint classification . . . . . . . . . . . . . . . . . . 28

3.1 .2 Various approaches for classification . . . . . . . . . . . . . . . . . . . . 29

3.2 Novel use of minutiae for classification . . . . . . . . . . . . . . . . . . . 30

3.2 .1 Minutiae based classification algorithm . . . . . . . . . . . . . . . 31

3.3 Analyzing classifier performance . . . . . . . . . . . . . . . . . . . . 43

4 Reconstruction of fingerprints

4.1 Synthetic fingerprint generation . . . . . . . . . . . . . . . . . 45

4.2 Reconstructing fingerprints from minutiae points . . . . . . . . . . . . . . . . . 51

4.2 .1 Reconstructing fingerprints using Gabor-like filters . . . . . . . . . . . . . 52

$4.2 .2 \quad$ Reconstructing fingerprints using Streamlines and LIC . . . . . . . . . . . 58

4.2 .3 Comparing two reconstruction schemes . . . . . . . . . . . . . . . 66

4.2 .4 Spoofing of fingerprint authentication system . . . . . . . . . . . . . 68

$\begin{array}{lll}5 & \text { Summary and Future work } & 73\end{array}$

5.1 Future work . . . . . . . . . . . . . . . . . . . 75

$\begin{array}{ll}\text { References } & 77\end{array}$ 


\section{List of Figures}

1.1 Various biometric identifiers. . . . . . . . . . . . . . . . . . . . . . 2

1.2 A typical biometric system. . . . . . . . . . . . . . . . . 3

1.3 (a) A fingerprint with ridge ending (E) and bifurcation (B) with position (x, y) and orientation $(\theta),($ b) Core and delta points. . . . . . . . . . . . 4

1.4 Various types of attacks on a biometric system (adapted from [1]) . . . . . . . . 7

1.5 Block diagram for Adler's face regeneration algorithm. . . . . . . . . . . . . . . 9

1.6 Block diagram of Hill's fingerprint regeneration algorithm. . . . . . . . . . . . . . 11

1.7 (a) Original Arch, (b) Reconstructed fingerprint from minutiae points using line

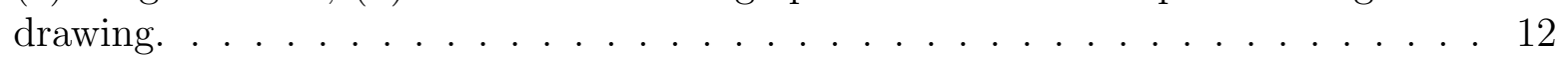

2.1 (a) A fingerprint image, (b) Corresponding orientation map. . . . . . . . . . . . . 15

2.2 Minutiae plots of 4 fingerprint classes: (a) A, (b) W, (c) L, and (d) R. . . . . . . . 16

2.3 A block diagram showing main steps of orientation estimation algorithm. . . . . . 17

2.4 A minutiae triplet. . . . . . . . . . . . . . . . . . . . . 17

2.5 Example of a minutiae triplet with large $\theta_{\text {diff }} . \ldots \ldots \ldots$. . . . . . . . . . . 19

2.6 Example of a triplet with large $L_{\max }$ for Arch and Whorl, (a) Correct result, (b) Incorrect result, (c) An example of skinny triple. . . . . . . . . . . . . . 20

2.7 (a) Contained triplets, (b) Overlapping triplets. . . . . . . . . . . . . . . 20

2.8 (a) Minutiae distribution of a fingerprint, (b) Examples of good (blue), with Lavg $=112.66$, Var $=5, \mathrm{Q}=237.63$, and bad (red) with $\mathrm{Lavg}=217$, Var $=26, \mathrm{Q}=$ 67.55, quality triplets, (c) Estimated orientation map. . . . . . . . . . . . 21

2.9 (a) Original Arch, (b) Triplet formation, (c) Orientation Estimation Result. . . . . 22

2.10 (a), (c) and (e) are original fingerprints of L, R and W whereas (b), (d) and (f) are their estimated orientation maps. . . . . . . . . . . . . . . 23

2.11 Comparing the estimated orientation map (from minutiae) with the true map: (a) Minutiae plot of a fingerprint, (b) Estimated orientation map, (c) True orientation map. Due to absence of valid triplets around the core region, the estimated orientation map cannot capture these regions, e.g., the concentric pattern in whorls. 25

2.12 Correlation between $\boldsymbol{\theta}$ and $\theta$ as observed on the NIST 4 database. About $79 \%$ of the orientation pairs have correlation more than 0.75 . . . . . . . . . . . . . 26

3.1 Five classes of fingerprints according to Galton-Henry classification scheme [2]. Core is represented by a square whereas the delta by a circle. . . . . . . . . . 27

3.2 Minutiae plots of 4 fingerprint classes: (a) A, (b) W, (c) $\mathbf{L}$, and (d) R. . . . . . . 31

3.3 Block diagram showing main steps of minutiae-based classification algorithm. . . . 32

3.4 (a) and (b) show $\mathbf{L}$ and $\mathbf{W}$ fingerprints divided into various areas whereas (c) and (d) show the corresponding original fingerprints respectively. . . . . . . . . . . . 32 
3.5 (a) Circular plot of minutiae, (b) Line ' $\mathrm{L}$ ' is perpendicular to the orientation of minutiae $\mathrm{m}$, (c) L made nearly perpendicular $\left( \pm 30^{\circ}\right)$ to the orientation $\theta$. . . . . 33

3.6 Minutiae plot overlaid on original fingerprint of $\mathbf{A}, \mathbf{L}$ and $\mathbf{W}$ are shown in (a), (c) and (e) respectively. The corresponding $R_{0}$ points marked in blue ('X') detected using estimated orientation maps are shown in (b), (d) and (f). . . . . . . . . 35

3.7 (a) The orientation map and detected registration point marked in blue ( $\left.{ }^{(*)}\right)$, (b) Salient minutiae in a $300 \times 300$ frame about $R_{0} . \ldots \ldots \ldots$. . . . . . . . 36

3.8 Rose plots of (a) $\mathbf{A}$, (b) $\mathbf{W}$, (c) $\mathbf{L}$, (d) $\mathbf{R}$. . . . . . . . . . . . . . . . . . 37

3.9 A whorl minutiae plot showing 4 types of minutiae pairs. . . . . . . . . . . . . 38

3.10 Minutiae density associated with 4 different classes of fingerprints: (a) A, (b) W, (c) L, and (d) R. These plots were generated using 30 images per class. Blue indicates a low density region while red indicates a high density region. . . . . . . 39

3.11 Kernel for (a) $\mathbf{L}$, (b) $\mathbf{R}$, and (c) W. . . . . . . . . . . . . . . . . . . . . 40

3.12 Red Vector: Unit tangent vector to the kernel, Green Vector: Direction of flow field at point $\gamma_{t}[3] \ldots \ldots \ldots \ldots \ldots \ldots$

3.13 Small inter-class variability between classes $\mathbf{L}$ and $\mathbf{T}$ (a) $\mathbf{R}$ and $\mathbf{W}$, (b) and large intra-class variability between two prints of whorl class (c) and (d). . . . . . . 43

3.14 Atypical minutiae plot of a fingerprint of $\mathbf{R}$ class. . . . . . . . . . . . . . . . . . 44

4.1 The process used by SFINGE to generate fingerprints: (a) Fingerprint shape generation, (b) Orientation image for a given set of core and delta points, (c) Output white image initialized with random seeds, (d) Generation of ridge pattern and minutiae initiated from seed points, (e) Synthetic L fingerprint, (f) Synthetic fingerprint with noise. (All figures are generated using SFINGE software). . . . . . . 50

4.2 Block diagram showing the main stages of our reconstruction algorithm. . . . . . . 53

4.3 (a) Image block initialized with seeds, (b) A Gabor filter tuned to $45^{\circ}$, (c) Generated ridge pattern, (d) Image block initialized with seeds, (e) A Gabor filter tuned to $191^{\circ}$, (f) Generated ridge pattern. . . . . . . . . . . . . . . . 54

4.4 Fingerprint reconstruction: (a) Estimated orientation field for a $\mathbf{L}$ fingerprint, (b) Ridge generation after 1 iteration, (c) Ridge generation after 10 iterations, (d) Reconstructed ridge pattern after 25 iterations, (e) Original L fingerprint. . . . . 55

4.5 (a) Estimated Orientation Map $\boldsymbol{\theta}$, Output of ridge generation with (b) $\sigma=0.2$, (c) $\sigma=1.2$, (d) $\sigma=2$, (e) Original fingerprint for classes $\mathbf{A}, \mathbf{L}, \mathbf{R}$ and $\mathbf{W}$. . . . 56

4.6 Output of ridge generation with (a) $D=0.1$, (b) $D=0.2$, (c) $D=1 / \sigma$, (d) $D=5$, (e) $D=10$, (f) $D=20 . \ldots \ldots \ldots \ldots \ldots$

4.7 (a) Estimated orientation map $\hat{\boldsymbol{\theta}}$, (b) Reconstructed fingerprint, (c) Original fingerprint of $\mathbf{T}, \mathbf{R}$, and $\mathbf{W}$ respectively. . . . . . . . . . . . . . 57

4.8 Block diagram showing visualizing vector field using LIC. . . . . . . . . . . . . . . 59

4.9 Block diagram showing the main stages of the proposed fingerprint reconstruction algorithm. . . . . . . . . . . . . . . . . . . 59

4.10 (a) A streamline $S$ tangent to orientation field $\hat{\boldsymbol{\theta}}$, (b) Streamline originating from a seed point (x,y) in the vector field $\boldsymbol{\theta}$

4.11 (a) Estimated orientation map $\boldsymbol{\theta}$ with border points marked in 'red' color, (b) Streamlines generated from minutiae points as seed points, (c) Streamlines generated using border points as seed points for classes, (d) Original fingerprint for $\mathbf{A}$, $\mathbf{R}, \mathbf{L}, \mathbf{W}$ respectively. . . . . . . . . . . . . . . . . . . . 
4.12 (a) Lattice of seed points $\left(D_{\text {seed }}=10\right)$, (b) Seed points 'S' overlaid on the orientation field, $\boldsymbol{\theta}$, of an arch, (c) Streamline terminated near a minutia 'm'. . . . . . 63

4.13 (a) Estimated orientation map $\boldsymbol{\theta}$, (b) Streamlines generated without controlling $D_{r}$, (c) Streamlines generated by controlling $D_{r}=5$, for $\mathbf{A}, \mathbf{R}, \mathbf{L}, \mathbf{W}$. . . . . . . . 64

4.14 (a) Minutiae plot, (b) Estimated orientation map $\boldsymbol{\theta}$, (c) Streamlines . . . . . . . . 65

4.15 Lending texture to a streamline using LIC: (a) White noise image, T, (b) Convolving T with a streamline, (c) Result of LIC. . . . . . . . . . . . . . 66

4.16 (a) Estimated orientation map $\boldsymbol{\theta}$, (b) Result of applying LIC, (c) Enhanced fingerprint using a $5 \times 5$ lowpass filter, (d) The original fingerprint. . . . . . . . . 67

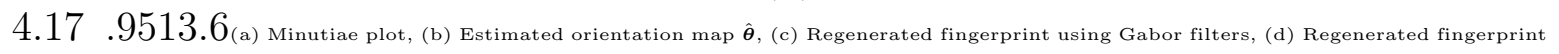
using LIC $\ldots \ldots \ldots \ldots \ldots$

4.18 .9513 .6 overlay of original (magenta) and reconstructed (blue) fingerprints for two minutiae templates. $\ldots \ldots \ldots 8$

4.19 (a) Original fingerprint and its minutiae plot, (b) Estimated orientation map $\hat{\boldsymbol{\theta}}$, (c) Result of applying LIC, (d) Enhanced ridge structure using COTS. . . . . . . 69

4.20 .9513.6 $6_{\mathrm{CMC}}$ curves when class of reconstructed fingerprint is (a) unknown (matched against all fingerprints of NIST-4f database), (b) known (matched against all fingerprints of the same class only). . . . . . . . . . . . . . . . . . . . . . . . . 70

$4.21 \quad 9513.6_{\mathrm{CMC}}$ curves when reconstructed fingerprint matched against all the fingerprints in FVC2002 DB3 database). $\ldots . . .71$

4.22 Estimated orientation map $\hat{\boldsymbol{\theta}}[(\mathrm{a}),(\mathrm{d}),(\mathrm{h})]$, Reconstructed fingerprint [(b), (e), (i)], Enhanced image by VeriFinger and its extracted minutiae [(c), (f), (j)], Original fingerprint $[(\mathrm{d}),(\mathrm{g}),(\mathrm{k})]$ for 3 fingerprints of DB3 FVC2002 database. . . . . . . . 72

4.23 (a) Estimated orientation map $\boldsymbol{\theta}$, (b) Reconstructed fingerprint, (c) Enhanced image by VeriFinger and its extracted minutiae, (d) Original A fingerprint of NIST $4 f$ Database. . . . . . . . . . . . . . . . . . . . . . . 72

5.1 Reconstructing fingerprints using hill-climbing approach. . . . . . . . . . . . . 76 


\section{List of Tables}

3.1 Number and position of singularities in five fingerprint classes. . . . . . . . . . . . 28

3.2 A summary of different fingerprint classification techniques. . . . . . . . . . . . . . 30

3.3 Confusion matrix indicating classification performance. . . . . . . . . . . . . . . 42 


\section{Chapter 1}

\section{Introduction}

\section{$1.1 \quad$ Biometrics}

Biometrics refers to the automatic recognition of a person based on his/her physical (e.g., fingerprints, face, iris, retina, voice, etc.) or behavioral (e.g., signature, gait, etc.) characteristics. A biometric system is primarily a pattern recognition system, that uses identifiers like fingerprints, iris, voice, hand geometry, etc. (Figure 1.1) to establish the identity of a person [4]. The primary goal of biometrics in authentication systems is to provide identity assurance, or the capability to accurately recognize individuals, with greater reliability, speed and convenience at lower costs. In the past few years, there has been an exponential growth in the use of biometrics in user authentication applications because it offers several advantages over traditional token-based or password-based systems, such as:

1. It uses 'something you are' rather than 'something you have or something you remember'. Thus, unlike traditional token-based or password-based authentication methods, biometric identifiers cannot be easily stolen, forgotten or shared. This makes them more reliable.

2. The traditional token-based or password-based authentication methods cannot determine if the person possessing the token is the same one who is enrolled in the system. As the biometric identifiers are unique across individuals, this problem can be addressed in case of biometric systems.

3. They are convenient to use. For example, a face recognition system installed in an ATM requires just a snap shot of the face to perform a transaction. 


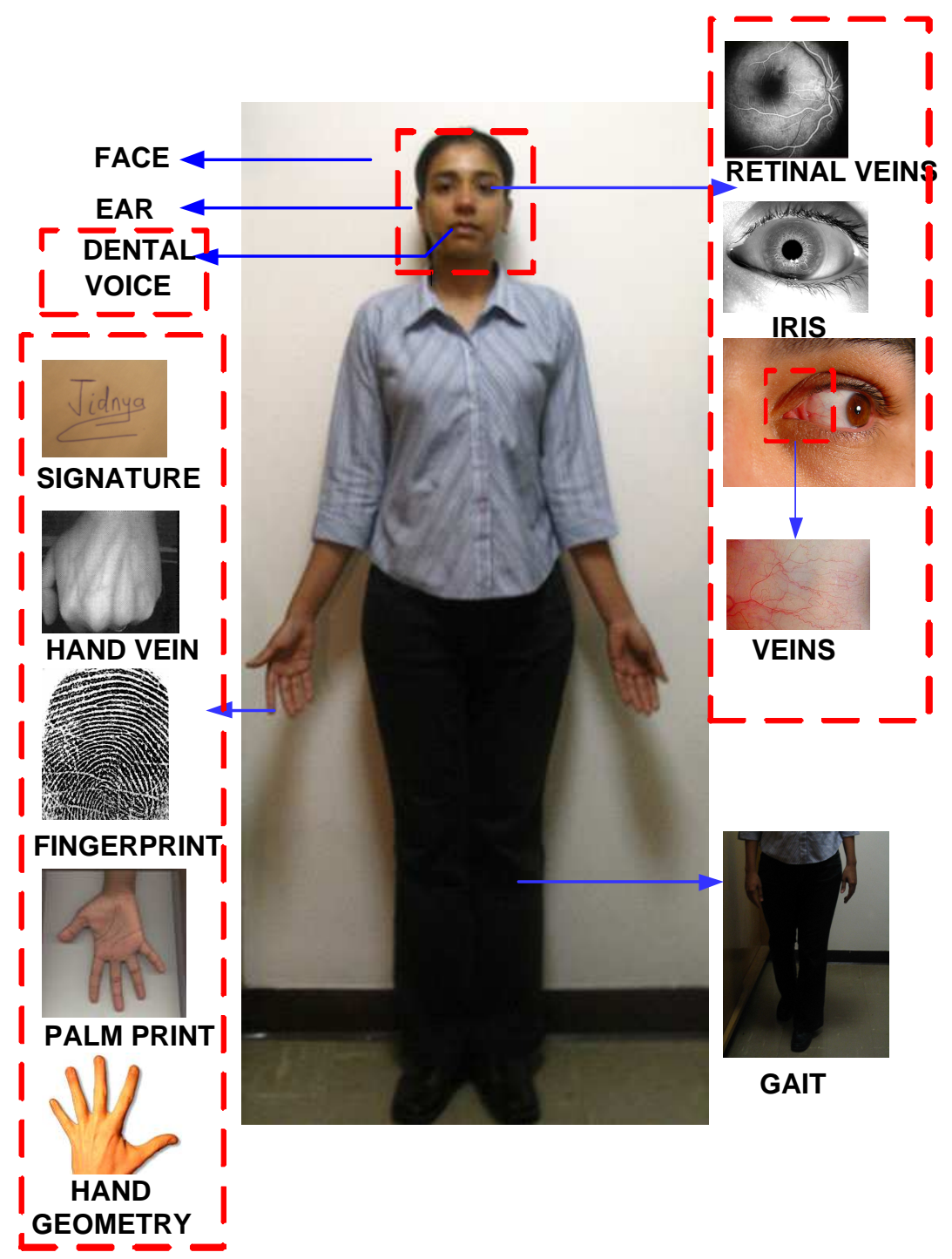

Figure 1.1: Various biometric identifiers. 
Biometric authentication can be used to control the security of banking transactions, personal computers, cell phones, web access, electronic commerce, restricted premises like nuclear plants, etc. Also it has recently been used at US airports for verifying the identity of the frequently flying individuals at the time of immigration.

A typical biometric authentication system is illustrated in Figure 3.1. It performs the following processing steps:

1. acquires a biometric sample,

2. extracts salient feature set (biometric template) and stores in a database,

3. compares a "live" template to previously stored templates, to calculate a match score [5].

The enrollment stage comprises of storing the template of each user in the database. Depending on the application, a biometric system can be used for verification or identification. Identification is also called as "one-to-many" matching. The system identifies an individual by comparing his feature set with all the templates stored in the database. Verification refers to "one-to-one" matching. Here an individual claims to be an enrolled user. To verify this claim, his template is compared with the stored template of the claimed identity.

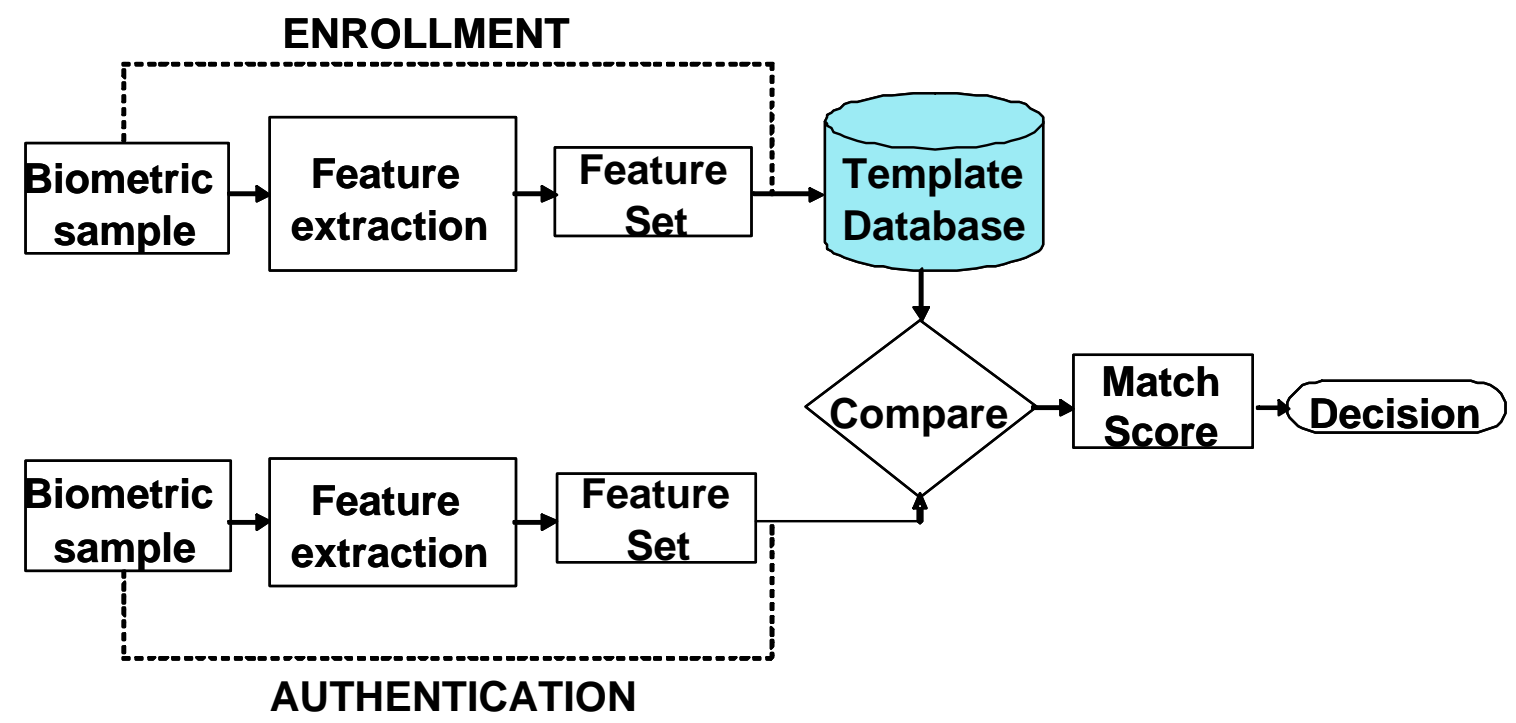

Figure 1.2: A typical biometric system. 


\section{$1.2 \quad$ Fingerprints}

Among all known biometrics like iris, face, speech, hand geometry, etc., fingerprint is one of the oldest and widely recognized biometric trait due to its attractive properties like permanence and individuality. Fingerprints have been used in law enforcement for many years.

A fingerprint is a smoothly flowing pattern of alternating ridges and valleys. The ridges present on the skin of the finger which makes contact with an incident surface under normal touch are called as friction ridges [6]. The unique pattern formed by these friction ridges forms a fingerprint. These ridges do not flow continuously but rather display various types of imperfections known as minutiae (minor details in fingerprints). The various types of minutiae are: ridge ending, ridge fork, island, dot, broken ridge, bridge, spur, enclosure, delta, double fork, bifurcation, trifurcation, etc. In all 150 minutiae types have been reported in the literature [7]. Out of all these, bifurcations and ridge endings are the most stable and robust points in fingerprints [4]. The point at which a ridge terminates or, alternatively, begins is called as ridge ending. A ridge ending is surrounded on three sides by valley. And the point at which a ridge splits into two ridges or, alternatively, where two separate ridges combine into one is called as bifurcation (See Figure 1.3 (a)). At the time of enrollment in a fingerprint system, important minutiae information (typically positions of ridge endings and bifurcations and the associated orientations) is extracted and stored in the database in the form of a template. A fingerprint

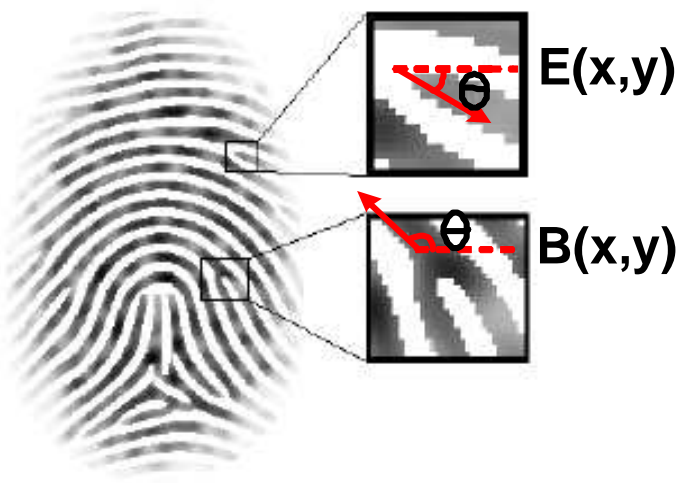

(a)

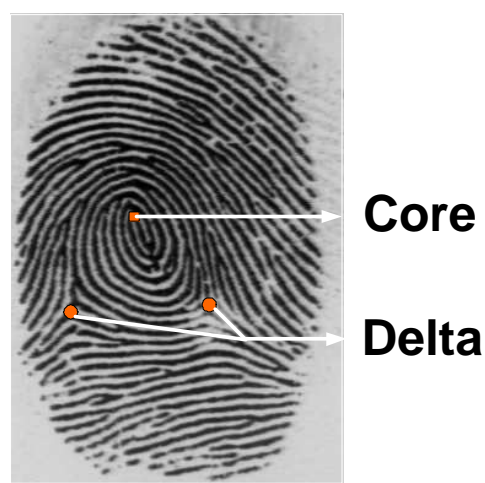

(b)

Figure 1.3: (a) A fingerprint with ridge ending (E) and bifurcation (B) with position (x, y) and orientation $(\theta)$, (b) Core and delta points.

can be also represented using two global features namely core and delta. These are also called are called singular points. The core point is defined as the highest point on the innermost ridge, 
while, the delta point is defined as the point where ridge flows having three different directions meet as shown in Figure 1.3 (b). As minutiae representation is stable and comparatively easy, most of the automatic fingerprints matching systems are only minutiae based. Every minutiabased fingerprint authentication system stores minutiae information in a template and uses it for authentication. The simplest template includes minutiae position, their type and respective orientation. The position of a minutia is given by its $\mathrm{x}$ and $\mathrm{y}$ coordinates while its orientation, $\theta$ is defined by the angle between the associated ridge and the horizontal axis.

The pattern of minutiae distribution in a fingerprint forms a valid representation of the fingerprint, since it is observed to vary across individuals (and across fingers of the same individual) [8]. Typically, in a live-scan fingerprint image of good quality, approximately 40-60 minutiae $(500 \times 500$ image size $)$ are obtained [4]. Fingerprint matching is accomplished by comparing the minutiae distribution of two fingerprints via sophisticated point pattern matching techniques [9].

"Two like fingerprints would be found only once 10 raised to 48 years." [8]. Although fingerprints have strong history of being used extensively in forensics since many years, there have not been any scientific evidence for their distinctiveness, i.e., individuality. It is generally accepted due to empirical results. By individuality, we mean that given a target population of the fingerprints, the probability of getting a sufficiently similar fingerprints is very small [10]. Along with overall ridge flow pattern, ridge frequency and location of singularities, minutiae points have been used extensively in individuality models. Various properties of minutiae like location, direction, type, ridge counts between pairs of minutiae, etc., contribute to fingerprint individuality.

Normally, templates will only contain information necessary for comparison. However, it is not fixed what is necessary for comparison. American National Standard for Information Technology (ANSI) proposed a standard, "Fingerprint Minutiae Format for Data Interchange", ANSI/INCITS 378-2004, that specifies data format for minutiae in fingerprints [6]. The standard contains definitions of relevant terms, a description of where minutiae shall be defined, a data format for containing the data, and conformance information. This standard facilitates inter-operability and efficiency in terms of storage for minutiae-based fingerprint templates. According to this standard, a minutiae template may contain:

1. position of minutiae $(\mathrm{x}, \mathrm{y})$,

2. orientation of minutiae $(\theta)$, 
3. type of minutia (bifurcation or ridge ending),

4. quality of minutiae,

5. ridge count information for the 8 neighbor-octants surrounding each minutia

6. positions (x, y) and orientations $(\theta)$ of core(s) and delta(s).

In this thesis, our goal is to see how much information does the minutiae template reveals about the parent fingerprint. For our experiments, we assume the template contains minimum minutiae information (i.e., only minutiae position and orientation). As the minutiae templates are eventually used for matching, they are assumed to be of good quality.

\subsection{Vulnerabilities of a biometric system}

Despite having numerous advantages, biometric systems are vulnerable to various types of attacks that can decrease their security. These attacks have been identified and examined in [11, 12, 13]. Ratha et al. [1] have identified eight basic sources of attack on a biometric system. They are illustrated in Figure 1.4. These attacks can be generalized for any biometric system.

1. Spoofing using fake biometric: In this type of attack, the hacker spoofs the biometric system by providing fake biometric samples to the biometric sensor as shown in Figure 1.4. Examples include a gummy finger (made from silicon rubber), a face mask, etc.

2. Resubmitting old biometric sample (Replay attack) : In this attack, the hacker bypasses the biometric sensor and provides an previously acquired copy of biometric signal to the feature extractor. This is called as a replay attack.

3. Overriding feature extractor: The hacker attacks the feature extractor to make it produce the desired features.

4. Altering extracted features : Here it is assumed that the hacker knows the representation of features that are extracted by the feature extractor. He replaces the original feature set with the synthetic feature set. Uludag and Jain [13] presented such an attack on a fingerprint based authentication system that uses hill climbing attack to synthesize the target minutia templates in order to achieve positive identification. This is a very difficult attack as generally, the feature extractor and matcher are integrated. 


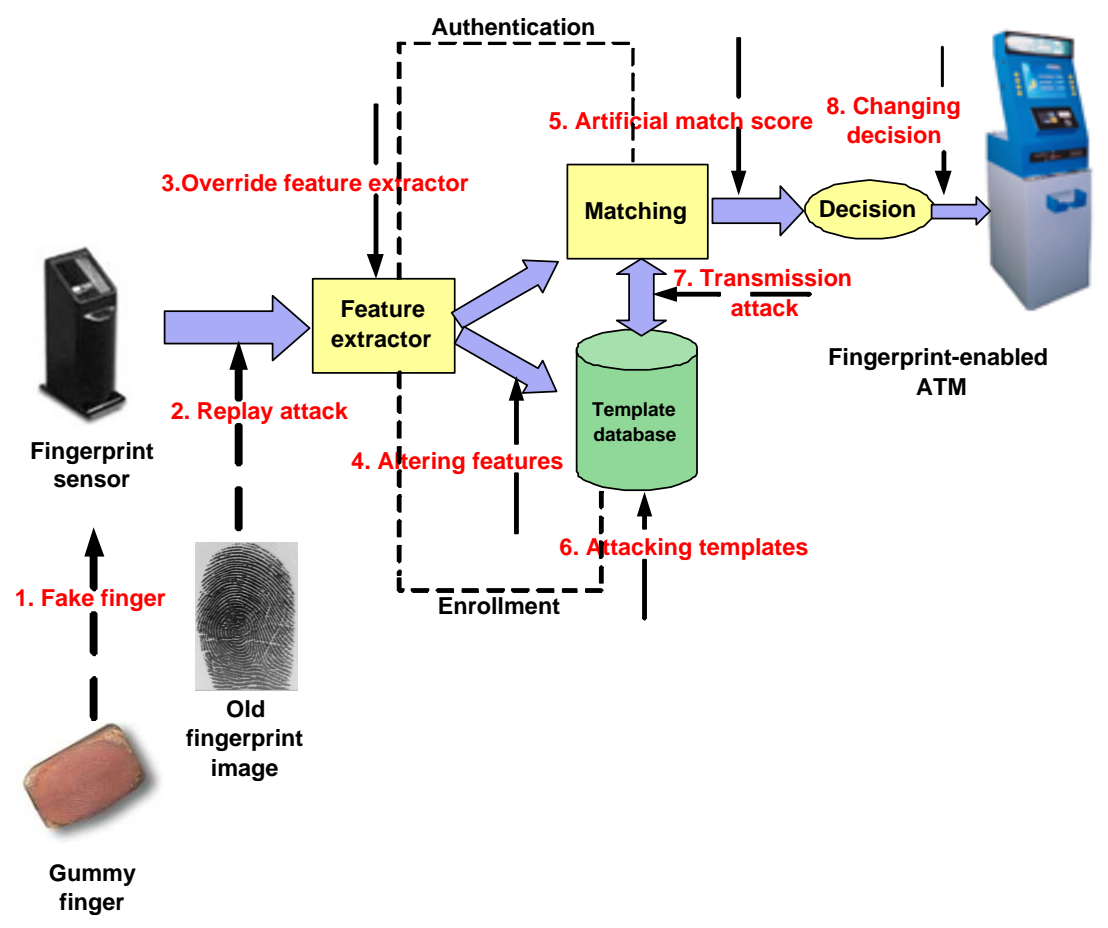

Figure 1.4: Various types of attacks on a biometric system (adapted from [1]).

5. Altering match score: Here, the matcher is attacked to directly produce the artificially high or low match score.

6. Tampering with stored templates: During enrollment, the biometric templates are stored in the database. At the time of authentication, this database can be available locally or remotely. The attacker attacks this template database and modifies the stored templates which could help him in authorization fraudulently.

7. Transmission attack: This attack occurs during transmission of templates from the database to the matcher. The hacker can modify the templates during this transmission before they reach matcher in order to achieve the desired score.

8. Changing decision : Being at the decision level, this can be a very dangerous attack. Although other components of the biometric system such as scanner, feature extractor, are performing outstandingly, if the hacker overrides the final decision, the performance of biometric system fails drastically. 


\subsubsection{Vulnerability of a biometric template}

Traditionally, a biometric template is not expected to reveal any significant information about the original biometric data. Thus it has been always considered to be a non-identifiable data [14]. Several minutiae-based fingerprint systems have denied the possibility that the stored templates could be used to generate implicit fingerprint information. 1 This is because,

1. the template does not contain the entire biometric sample but has only essential features, e.g. minutiae in case of fingerprints. The size of template is much smaller than the original biometric sample,

2. during enrollment procedure, there is loss of information about the acquired biometric sample due to image scanning, pre-processing, feature extraction, and template creation. For example, during minutiae extraction from a fingerprint image, a fingerprint image undergoes finalization and skeletonisation procedures. During this procedure, significant information of the original fingerprint may be lost, and

3. the storage format of the templates makes it difficult to "hack".

Thus till recently, biometric template generation algorithms were considered to be one-way algorithms. But Adler [14], from Ottawa University, demonstrated that the original face images can be regenerated from face recognition templates using only the match score values. Also, Hill [15] demonstrated the masquerade attack on a fingerprint matching system by reconstructing original fingerprints from their stored minutiae templates. The details about both the techniques are discussed below.

\section{Face image regeneration}

Adler proposed a simple algorithm to regenerate face images with the help of a face authentication system. He assumes that the face authentication system releases a match score for each authentication. His technique begins with a guess of the target face, makes small modifications;

\footnotetext{
${ }^{1}$ All web-sites accessed in August 2005.

- http://www.biometricaccess.com/support/bacfaq09.htm: A true fingerprint image cannot be created from master template..

- http://www.digitalpersona.com/support/faqs/privacy.php: ... fingerprint templates cannot be used to recreate the fingerprint image.
} 
keeps modifications which increase the match score. This type of attack is called as hill climbing attack. A more sophisticated description of hill climbing attacks has been given by Soutar [16].

Adler's iterative procedure continues until it generates an image that gives a sufficient match score against the target face image. The main steps in the algorithm are shown in Figure 4.14 .

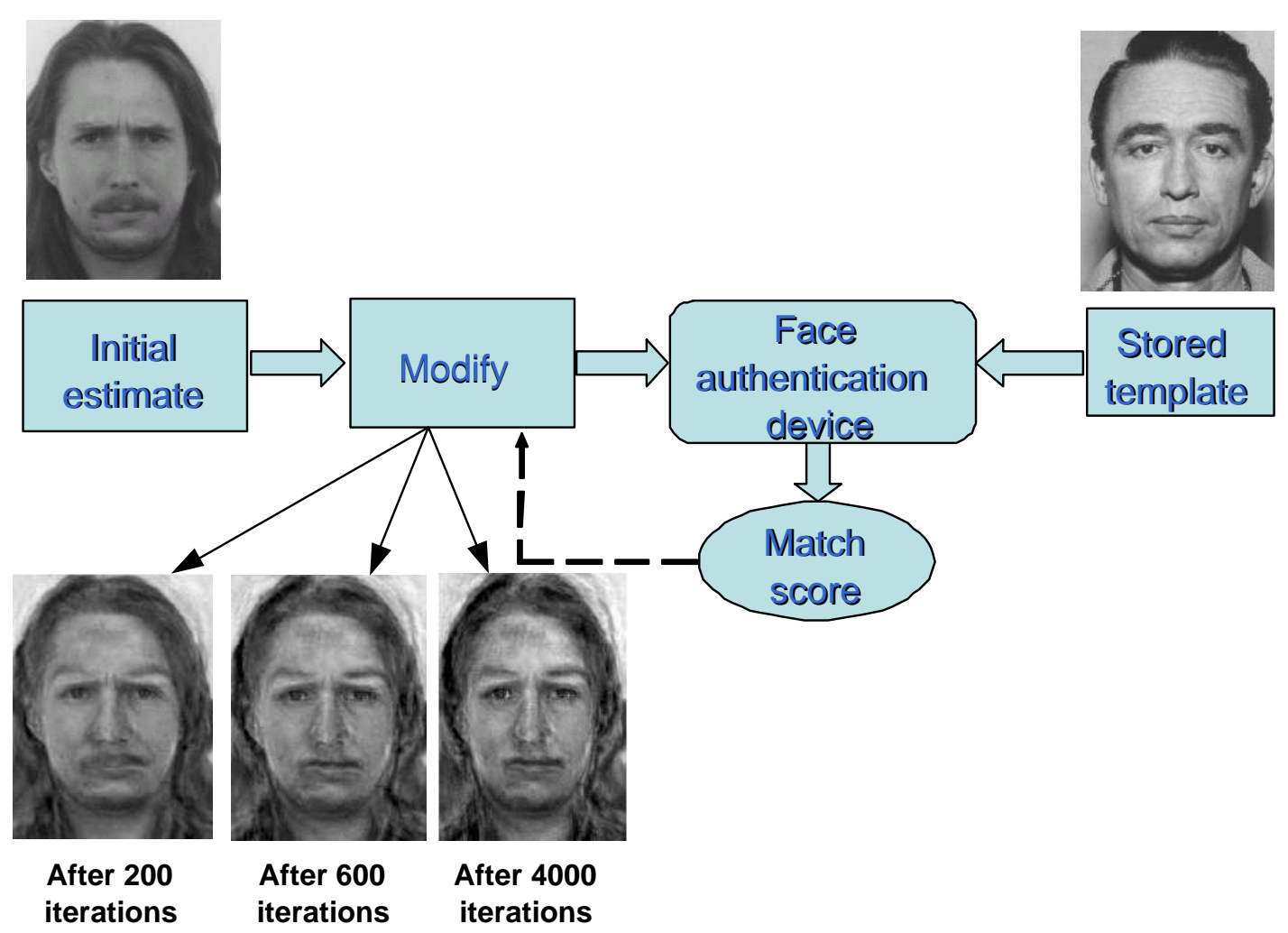

Figure 1.5: Block diagram for Adler's face regeneration algorithm.

The algorithm is a three-step procedure, which consists of preprocessing, initial image selection, and image estimate improvement. Adler demonstrated results using the University of Aberdeen face recognition database.

1. Preprocessing: The face images from the database are rotated, cropped, and histogram equalized so that all images have same size and distribution of pixel intensities. The eigen face decomposition of these images is accomplished.

2. Initial image selection: Using the face authentication system, the match scores for a selection of images from the local database against the target template are observed. The image giving the highest match score is selected to be the initial estimate. 
3. Image estimate improvement: The initial estimate is modified by the algorithm to better match the target face image. In each iteration, a constant (heuristically determined) times Eigen face is added to the initial estimate and an authentication is tried. The match score against the target image is observed. The modification is kept if the score improves otherwise a different modification is tried. The iterations are repeated till there is no significant improvement in the matching score. Within thousands of iterations, a face image, which gives high match against the target template is regenerated.

This technique cannot only masquerade the target person, but also gives good visual impression of the persons face as shown in Figure 4.14. Thus these look alike images could be used to masquerade the target person or to identify him. As the face template contains significantly less data than the original template, exactly re-creating the target face image is not possible using this approach.

One interesting aspect of this regeneration procedure is, it is not necessary to know how the algorithm works but requires only the match score values. It is not sensitive to the choice of optimization algorithm, the initial image estimate, or the local face database. Also, it does not require special expertise to 'fool' the face authentication system. Any system which allows access to match scores effectively allows sample images to be regenerated in this way. Adler tested his algorithm using three recent face recognition products of well-known commercial vendors. Two of these vendors also participated in the Face Recognition Vendor Test (2002) [17]. Results show that after about 4000 iterations, a sufficiently large matching score is obtained, which corresponds to a very high $(99.9 \%)$ confidence of matching scores. The confidence was calculated as a sigmoidal function of the matching scores.

As a consequence, BioAPI Consortium [18] recommended that all biometric systems should use quantized match scores. But Adler [19] also presented a modified hill climbing attack to prove that images can be regenerated from quantized match scores implying that quantization of match scores does not prevent the regeneration process. This work suggests that biometric templates and biometric match scores should no longer be considered to as non-identifiable data. Although Adler demonstrated the masquerade attack for face recognition systems; this approach can be readily extensible to other biometric modalities as well [?]. 


\section{Fingerprint image regeneration}

Unlike Adler, Hill [15] uses only the stored fingerprint templates instead of the match scores for fingerprint image reconstruction. It regenerates the original fingerprint from the minutiae information, $\mathrm{x}, \mathrm{y}, \theta$, and singular points (core and delta) that are stored in the template. Once a fingerprint template is obtained, the algorithm executes a sequential procedure involving shape prediction, orientation map creation, and line drawing. The main steps of this algorithm are described in Figure 1.7.

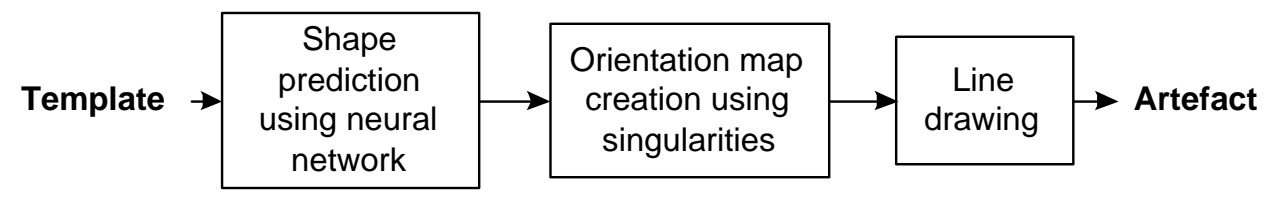

Figure 1.6: Block diagram of Hill's fingerprint regeneration algorithm.

1. Shape Prediction: Hill designed a decision tree for predicting the shape (class) of a fingerprint from stored information about singularities (core and delta) in the template. This decision tree is based primarily on the number of core points, and the relative positions of a delta point to a core point. If the singularities are not stored in the template then a neural network-based approach which uses just the minutiae $(\mathrm{x}, \mathrm{y}, \theta)$ information is employed. It consists of 23 input neurons, a single hidden layer of 13 neurons and an output layer corresponding to the 4 classes of the fingerprint (Arch, Left loop, Right loop and Whorl). Hill obtained a classification accuracy of $71 \%$ for a database of 242 fingerprints.

2. Orientation Map Creation: For fingerprint reconstruction, Hill assumes that the minutiae template stores information about singularities. Given the number and position of core and delta, he uses the orientation model proposed by Sherlock and Monro [20]. This technique relies on the presence of core and/or delta points. Various orientation maps were created assuming various possibilities of core and delta positions. An orientation map, which best suits the known minutiae points and predicted fingerprint shape is selected among the generated ones.

3. Line Drawing: For synthesis of fingerprint images, Hill uses line drawing approach. The algorithm draws lines from each known minutiae. It is an iterative procedure that continues 
till lines are drawn from all the minutiae points. The direction of the ridge is determined by consulting the direction map for the current position. Some extra lines of varying widths are added from the edges of the images for giving it appearance of the real fingerprint. The results of reconstruction are demonstrated only for Arch class. Also, the regenerated image do not have an appearance of true fingerprint. The results for reconstruction are shown in Figure 1.7. He compared 25 reconstructed fingerprints against the original ones using a fingerprint matching system and obtained a sufficient match score. He mentions that his regenerated fingerprints visually did not appear like true fingerprints thus the masquerade attack will fail for the fingerprint authentication systems where visual inspection of fingerprints is also employed.

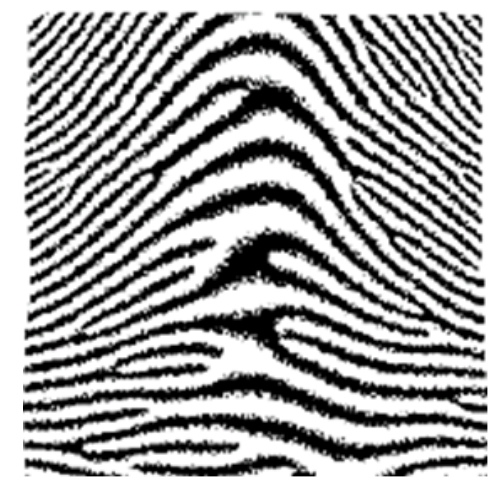

(a)

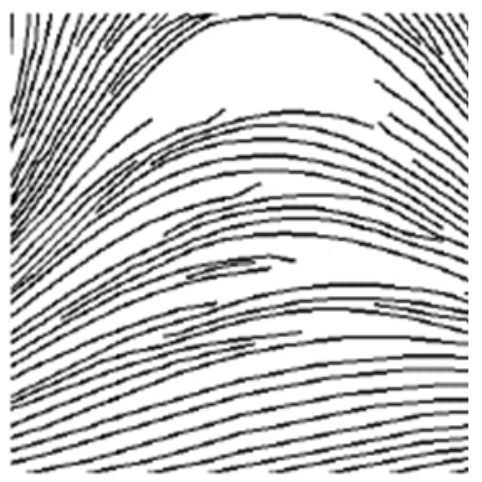

(b)

Figure 1.7: (a) Original Arch, (b) Reconstructed fingerprint from minutiae points using line drawing.

The implications of Hill's work was analyzed in a report by the International Biometric Group [21]. Three types of biometric image recreation were distinguished:

1. feature image (an image that bears little resemblance to the original biometric image but which still suffices to fool a biometric algorithm, rated achievable),

2. generic image (a rough resemblance to the original, rated very likely achievable), and

3. total image (virtually identical to the original, rated very difficult, though perhaps not impossible). 


\subsection{Problem Statement}

Most of the fingerprint-based authentication systems match two fingerprints by comparing their minutiae distribution. To achieve this, minutiae are stored in a database in the form of template. In this thesis, we concern ourselves to see how much information does a fingerprint template reveal about the parent fingerprint. This study will help to

1. understand the role of minutiae in fingerprint individuality models.

2. verify if we can use minutiae for classifying fingerprints.

3. confirm or challenge the traditional belief that it is safe to store minutiae information in the database.

The purpose of this thesis is not to demonstrate the masquerade attack on fingerprints rather it is just a consequence.

\subsection{Contribution of the Thesis}

In this thesis we demonstrate that several levels of information can be elicited from a simple minutiae template viz. the ridge orientations, class, and the ridge structure of the unseen parent fingerprint.

We first estimate the ridge orientations using the minutiae orientations to form a discrete orientation map. We observed that the estimated orientation map is consistent with the true ridge orientations. Then, using the estimated orientation map and the minutiae distribution, we designed a classifier to predict the class of the parent fingerprint. To our knowledge, this is the first time minutiae points have been used for fingerprint classification. Experiments conducted on the NIST-4 database indicate the efficacy of the proposed algorithm and suggests its potential in providing insights into the nature of the minutiae distribution for different classes of fingerprint.

Furthermore, we have proposed two techniques for fingerprint reconstruction. First technique uses Gabor-like space-invariant filters. This method does not have control over number and type of generated minutiae. Second technique uses two effective tools from flow visualization field namely streamlines and LIC (Line Integral Convolution). It allows generating minutiae at desired location in the regenerated fingerprint image. No literature was discovered during this 
research that describes a synthetic fingerprint generation technique to generate fingerprint with predetermined minutiae points. We further demonstrate that the generated fingerprints can be used to spoof a minutiae-based fingerprint authentication system.

\subsection{Organization of the Thesis}

Chapter 2 discusses a generic interpolation scheme for estimating orientations of underlying ridges based on minutiae information alone. Chapter 3 explains the design of a minutiae-based fingerprint classifier and illustrates the classification result for the NIST 4 fingerprint database. The fingerprint reconstruction schemes are discussed in Chapter 4. Finally, Chapter 5 summarizes the contribution of this thesis and considers the future work in this area. 


\section{Chapter 2}

\section{Estimating Ridge Orientations using Minutiae Points}

The ridge-line flow of a fingerprint can be effectively described by an orientation map which is a discrete matrix whose elements are the orientation of the tangent to the ridge lines. An example of orientation map for a fingerprint image is shown in Figure 2.1. This orientation

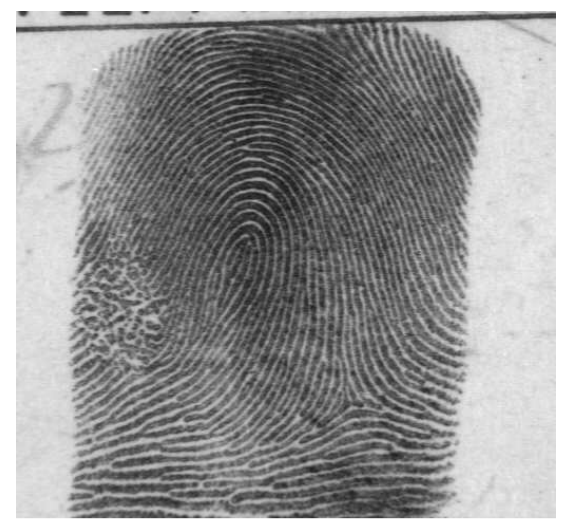

(a)

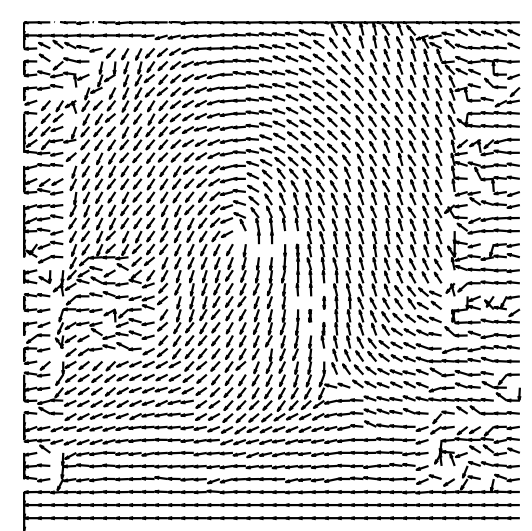

(b)

Figure 2.1: (a) A fingerprint image, (b) Corresponding orientation map.

map can be computed from the fingerprint image 2.1 (a) using various techniques proposed in the literature [22]. Our goal is to see if we can estimate the orientation map using only the minutiae points. The orientation of a minutia is an indication of the local ridge direction since the fingerprint is a smoothly changing oriented texture pattern [23]. Also, Stoney [8] stated that the minutiae orientations of a fingerprint represent its ridge orientations.

Consider the minutiae plots of four classes shown in Figure 2.2. These plots clearly suggest 


\section{CHAPTER 2. ESTIMATING RIDGE ORIENTATIONS USING MINUTIAE POINTS 16}

the possibility of deducing the direction of local ridges by examining the orientation of minutiae points in that region. The only information available in a minutiae template is the positions and orientations of minutiae points. Thus we utilize this information for estimating ridge orientations of the parent fingerprint. We consider the orientation of a local group of minutiae, in order to

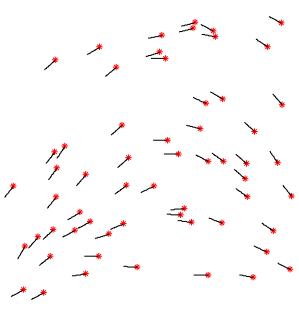

(a)

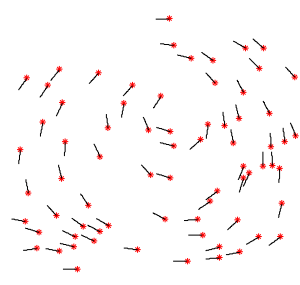

(b)

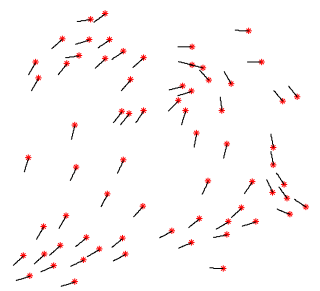

(c)

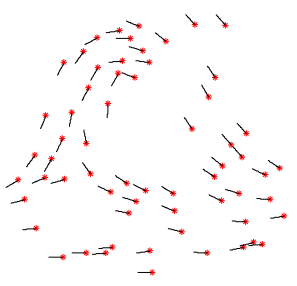

(d)

Figure 2.2: Minutiae plots of 4 fingerprint classes: (a) A, (b) W, (c) L, and (d) R.

'interpolate' the direction of the underlying ridge flow. We have proposed an algorithm that utilizes minutiae triplets to estimate the orientations of pixels present in the enclosed triangular regions. A triplet is formed using 3 minutiae as vertices. Kovacs - Vajna [24] have proposed a fingerprint verification technique that uses minutiae triplets. It was demonstrated that by using minutiae triplets for fingerprint matching, the relative nonlinear deformation present in the fingerprint image pairs was overcome. The triangular shape copes with the strong deformation of fingerprint images due to static friction or finger rolling. Also it saves local regularities and compensates for global distortion. Also, Germain et al. [25] proposed a fingerprint indexing technique using minutiae triplets. They proposed a similarity searching algorithm, Flash, akin to geometric hashing that utilizes minutiae triplets. They found that the minutiae triplets give some immunity against noise in fingerprints.

\subsection{Orientation Estimation Algorithm}

The proposed algorithm utilizes a minutiae triplet to estimate the orientation of a triangular fingerprint region defined by the triplet. If we go for more degrees of freedom (say a quadrilateral), although it would cover a larger fingerprint area, the estimated orientations may not be reliable for all the enclosed pixels. Also the triplet formed using minutiae as vertices are rotation and scale invariant. 
A minutia point can be represented as a three-tuple, $(\mathrm{x}, \mathrm{y}, \theta)$, where $(\mathrm{x}, \mathrm{y})$ is its spatial location and $\theta^{1}$ its orientation. The main steps of our robust orientation estimation algorithm are shown in Figure 2.3 .

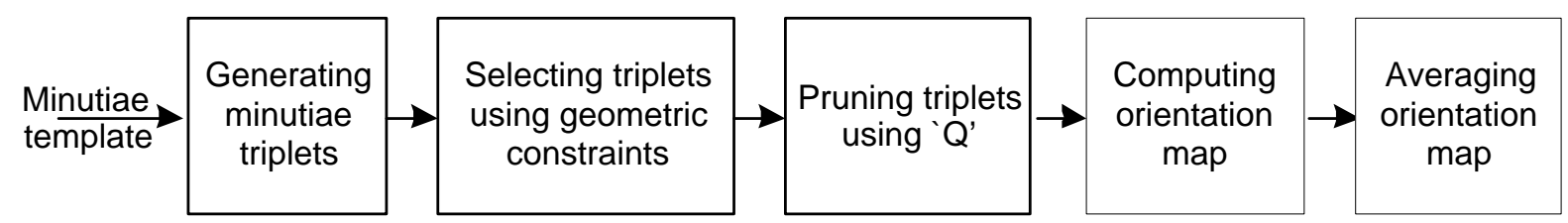

Figure 2.3: A block diagram showing main steps of orientation estimation algorithm.

1. Generating minutiae triplets: Consider a minutiae template, $M$, of a fingerprint containing $N$ minutiae points given by, $M=\left\{m_{1}, m_{2}, \cdots, m_{N}\right\}$ where $m_{i}=\left(x_{i}, y_{i}, \theta_{i}\right), i$ $=1$ to $N$. Figure 2.4 shows a triplet containing 3 minutiae, $m_{i}=\left(x_{i}, y_{i}, \theta_{i}\right), i=1,2,3$. The orientations of pixels enclosed by the triangular region can be estimated using the orientations of minutiae vertices. A set of 3 minutiae points, $m_{1}, m_{2}$, and $m_{3}$ is said to

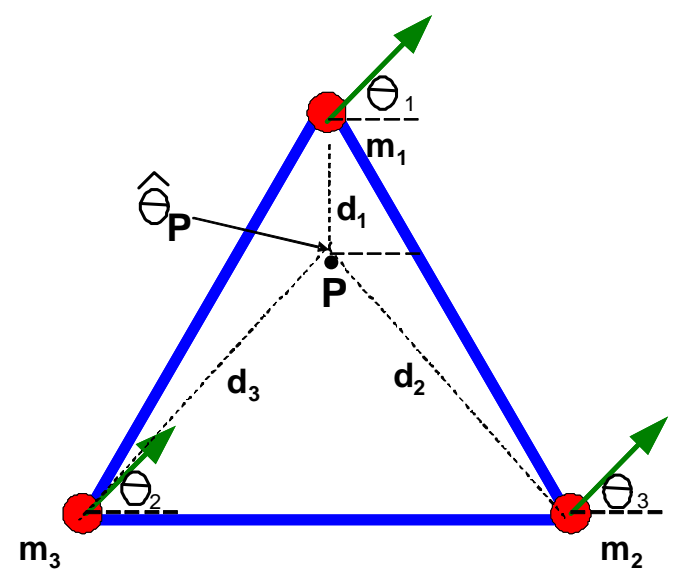

Figure 2.4: A minutiae triplet.

constitute a triplet, $T$, if $\operatorname{dist}\left(m_{i}, m_{j}\right) \leq 150$ (Euclidean distance), and $\left|\theta_{i}-\theta_{j}\right| \leq 15$, $\forall i, j=1,2,3$.

Let $P(x, y)$ be a point in the region enclosed by the triplet, and let $d_{i}$ be the Euclidean distance of $P$ from $m_{i}$ such that $d_{1}<d_{2}<d_{3}$. The orientation at point $P, \hat{\boldsymbol{\theta}_{\boldsymbol{P}}}(x, y)$, is

\footnotetext{
${ }^{1}$ We do not make distinction between opposing angles. i.e. minutiae having $30^{\circ}$ and $150^{\circ}$ orientations are treated the same. Thus the range of theta is $90^{\circ} \leq \theta \leq 270^{\circ}$
} 


\section{CHAPTER 2. ESTIMATING RIDGE ORIENTATIONS USING MINUTIAE POINTS 18}

then estimated by taking the weighted average of the 3 minutiae orientations:

$$
\hat{\boldsymbol{\theta}_{\boldsymbol{P}}}(x, y)=\frac{d_{3}}{\left(d_{1}+d_{2}+d_{3}\right)} \theta_{1}+\frac{d_{2}}{\left(d_{1}+d_{2}+d_{3}\right)} \theta_{2}+\frac{d_{1}}{\left(d_{1}+d_{2}+d_{3}\right)} \theta_{3}
$$

Here as $\mathrm{P}$ is more closer to $m_{1}, \theta_{1}$ will have more influence on $\boldsymbol{\theta}_{\boldsymbol{P}}$ than $\theta_{2}$ and $\theta_{3}$. So it gets more weight in equation 2.1. Generally for an image size of $512 \times 512$, there are $40-60$ minutiae [4]. Then the number of triplets that are possible can be given by,

$$
\left(\begin{array}{l}
n \\
3
\end{array}\right)=\frac{n !}{(n-3) ! 3 !}
$$

where, ' $\mathrm{n}$ ' is the number of minutiae in a fingerprint template. To keep the number of triplets generated within bounds, the algorithm employs certain geometric constraints while forming the triplets to ensure reliable orientation estimation. A triplet is selected only if it satisfies the following conditions.

\section{Selecting triplets using geometric constraints:}

(a) Orientation Difference $\left(\theta_{\text {diff }}\right)$ : The orientation of the fingerprint region inside the triplet is function of the orientations of minutiae (equation 2.1). Thus, all the minutiae vertices should have comparable orientations. For every triplet, we compute the orientation difference $\theta_{\text {diff }}$, given by,

$$
\theta_{\text {diff }}=\max \left(\theta_{i}-\theta_{\text {med }}\right), i=1,2,3
$$

where, $\theta_{\text {med }}$ is the median of three minutiae orientations. It should satisfy the condition,

$$
\theta_{\text {diff }} \leq \theta_{\text {tol }}
$$

where, $\theta_{t o l}$ is a predetermined threshold. In our experiments, we used $\theta_{t o l}=30^{\circ}$. This condition is employed to avoid triplets exhibiting large orientation differences. Such triplets may occur around core or delta region where there is large ridge activity. If these triplets are selected, we might get incorrect orientation estimation as shown in Figure 2.5.

(b) Maximum Length $\left(L_{\max }\right)$ : Let $L_{i}$ be the length of the side of a triplet, then all three sides of the triplet should satisfy the condition

$$
L_{\text {min }} \leq L_{i} \leq L_{\max }, i=1,2,3
$$




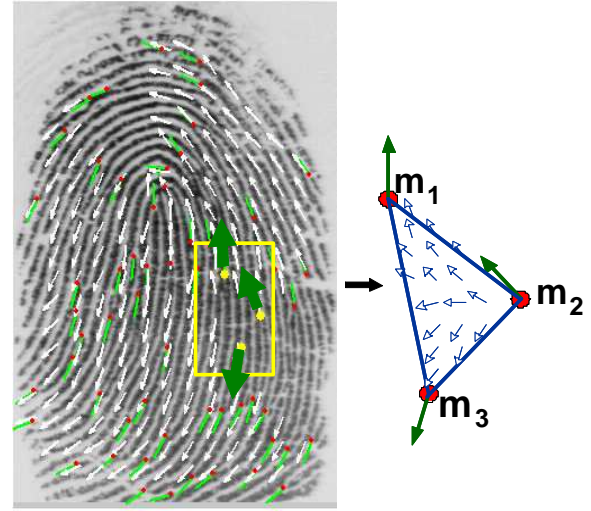

Figure 2.5: Example of a minutiae triplet with large $\theta_{\text {diff }}$.

where, $L_{\max }$ is the maximum length that can be allowed for a triplet.

A triplet enclosing a large fingerprint area, might have regions exhibiting changes in the ridge orientations. Thus, the orientation may not be estimated correctly for underlying larger fingerprint region. Hence, the triplets enclosing large fingerprint area need to be avoided. This is ensured by comparing the three side-lengths of minutiae triplet against a threshold.

Deciding a single value for $L_{\max }$ across all fingerprint classes is a difficult task. As shown in Figure 2.6 (a), due to parallel ridge flow in Arch, larger side triplet can also give reliable orientation estimation but in Whorls, due to the large ridge activity near core region, this is not always true. In order to have a generic algorithm for all fingerprint classes, this constraint is important. We used $L_{\max }=300$ for our experiments.

(c) Interior Angels $\left(\phi_{\min }\right)$ : Minutiae tend to occur in clusters [26]. In a cluster of minutiae, there may be some triplets that enclose very small fingerprint area as shown in Figure 2.6 (c). So using these triplets for estimating orientations is computationally inefficient. To avoid these triplets, the interior angles and side-lengths of the triplet are observed. A triplet is selected if,

$$
\begin{gathered}
\phi_{i}>\phi_{\text {min }}, \\
L_{i}>L_{\text {min }}, i=1,2,3
\end{gathered}
$$


where $\phi_{i}$ are interior angles of the triplet (Figure $2.6(\mathrm{c})$ ), $\phi_{\min }=20^{\circ}$, and $L_{\min }=20$ pixels. All three interior angles of a triplet should be greater than a preset threshold, $\phi_{\min }$, and also the minimum side-length should be greater than $L_{\min }$.

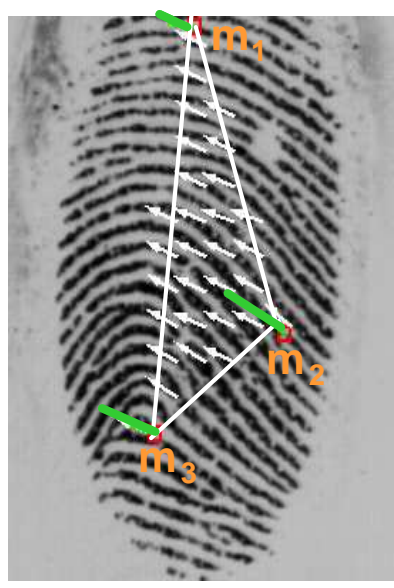

(a)

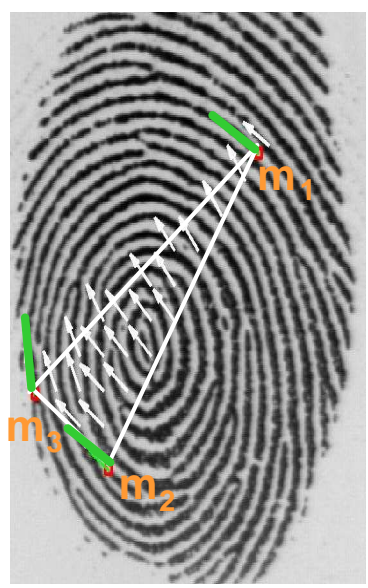

(b)

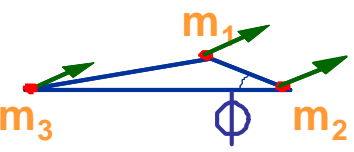

(c)

Figure 2.6: Example of a triplet with large $L_{\max }$ for Arch and Whorl, (a) Correct result, (b) Incorrect result, (c) An example of skinny triple.

3. Pruning triplets using Quality Factor: As mentioned above, fingerprints are characterized by clusters of minutiae in certain regions. For instance, the regions near the core and delta have dense minutiae activity. In such cases, a triplet may reside inside the triangular region of another triplet or may overlap with it. This results in multiple triplets enclosing common fingerprint area. In Figure 2.7 (a) one triplet is completely included in another

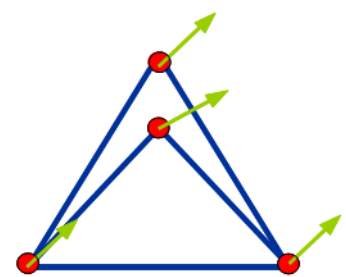

(a)

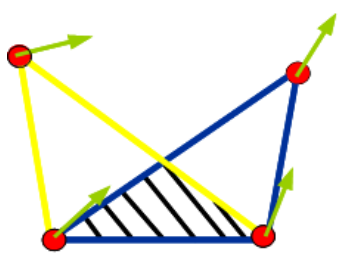

(b)

Figure 2.7: (a) Contained triplets, (b) Overlapping triplets.

whereas in Figure 2.7 (b) two triplets are overlapping. Here, rather than consolidating the orientation information estimated by multiple triplets, we utilize the information estimated only by a good quality triplet. To do so, a quality factor ' $Q$ ' is assigned to each triplet. 


\section{CHAPTER 2. ESTIMATING RIDGE ORIENTATIONS USING MINUTIAE POINTS 21}

The quality, Q, is measured by examining the average length of the sides of the triangle and the orientations of component minutiae points, and is computed as,

$$
Q=\left(L_{\max }-L_{a v g}\right) w_{1}+\left(\frac{\theta_{t o l}-\theta_{\text {diff }}}{\theta_{\text {diff }}} L_{\max }\right) w_{2} .
$$

Here, $L_{\text {avg }}$ is the average length of the sides of the triplet, $\theta_{\text {diff }}$ is the maximum difference between pairwise minutiae orientations (equation 2.3 ), and $w_{1}, w_{2}$ are the weights associated with each term. We use $w_{1}=0.4$ and $w_{2}=0.6$. This ensures that a triplet having minutiae of similar orientations and covering a relatively small area is assigned a higher $Q$ value. Thus in case of a tie, a triplet higher $Q$ value is selected. Examples of the good and bad quality triplets are shown in Figure 2.8.

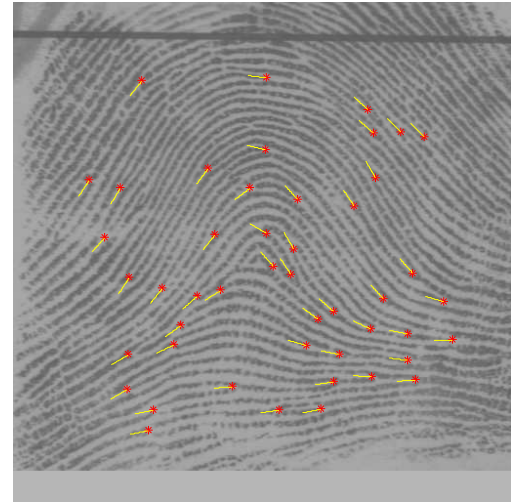

(a)

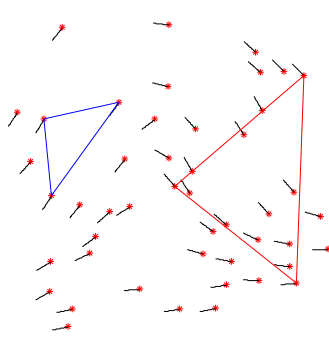

(b)

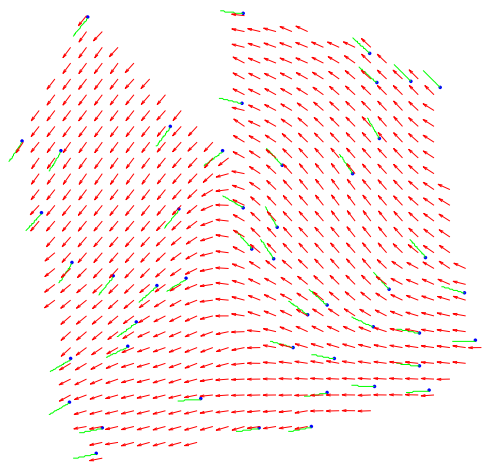

(c)

Figure 2.8: (a) Minutiae distribution of a fingerprint, (b) Examples of good (blue), with Lavg $=112.66$, $\operatorname{Var}=5, \mathrm{Q}=237.63$, and bad (red) with $\operatorname{Lavg}=217$, Var $=26, \mathrm{Q}=67.55$, quality triplets, (c) Estimated orientation map.

4. Computing orientation map $\hat{\boldsymbol{\theta}}$ : Using equation 2.1, orientations of the ridge pixels enclosed by valid triplets are estimated. An orientation map, $\hat{\boldsymbol{\theta}}$, is a matrix where each cell of this matrix represents the average orientation of a local window of the parent fingerprint image. For example, in a $512 \times 512$ image, if we take a local window size of $13 \times 13$, then an orientation map of size $39 \times 39$ is obtained by assigning the average orientation of the pixels in the local window to the corresponding cell in the orientation map. Some cells may not contain any orientation information due to the non-availability of 'valid' triplets in those regions. The estimated orientation map for an Arch fingerprint is as shown in Figure 2.9. 


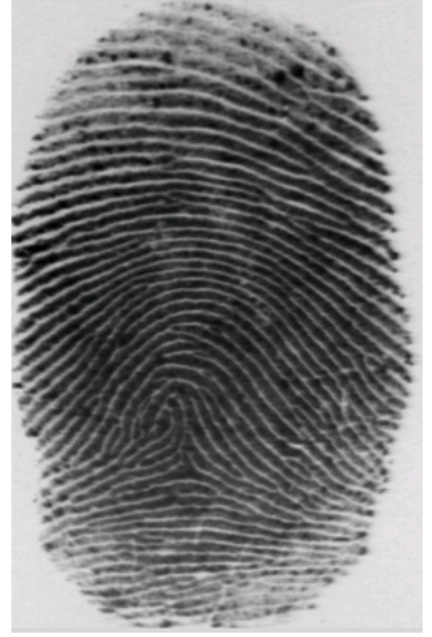

(a)

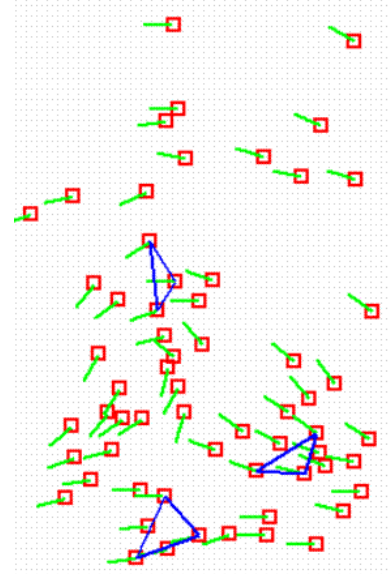

(b)

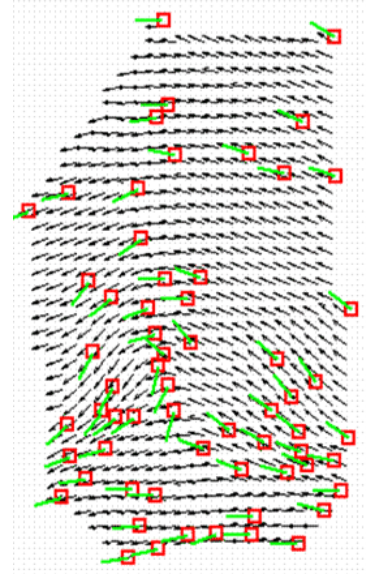

(c)

Figure 2.9: (a) Original Arch, (b) Triplet formation, (c) Orientation Estimation Result.

5. Averaging orientation map: To obtain a smooth transition in orientations, the estimated orientation map is convolved with a $3 \times 3$ local averaging filter.

Some results for estimated orientation maps for other fingerprint classes are shown in Figure 2.10 .

\subsection{Validating the estimated orientation map}

Visually, we observe that the estimated orientation map is quite consistent with the underlying ridge flow of the original fingerprint. In order to verify the accuracy of the algorithm, we compare the true orientation map with the estimated one using a correlation measure. The true orientation map can be computed using various techniques as described in [4]. We use the least mean square orientation estimation approach proposed by Ratha et al. [22] for computing the true orientation maps of fingerprint images. Given a fingerprint image I, the algorithm computes the ridge orientations at discrete points on the same cartesian grid used to compute $\hat{\boldsymbol{\theta}}$.

1. Divide I into $w \times w$ blocks (e.g., $13 \times 13$ ); the center of each block corresponds to a grid point. 


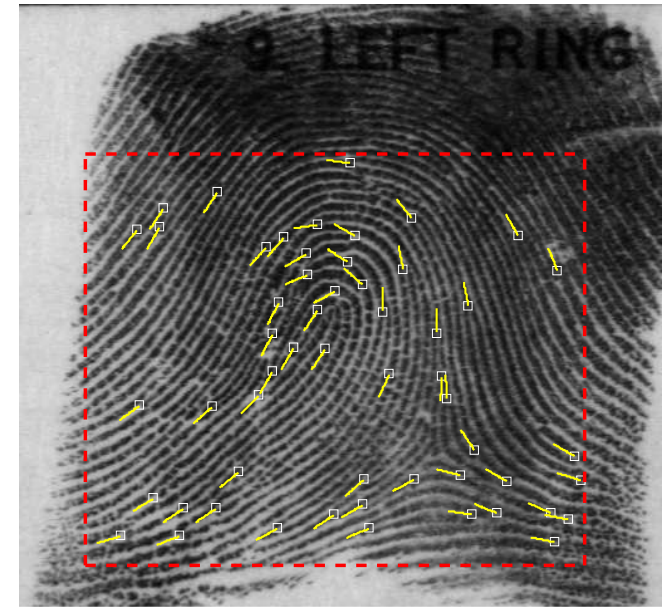

(a)

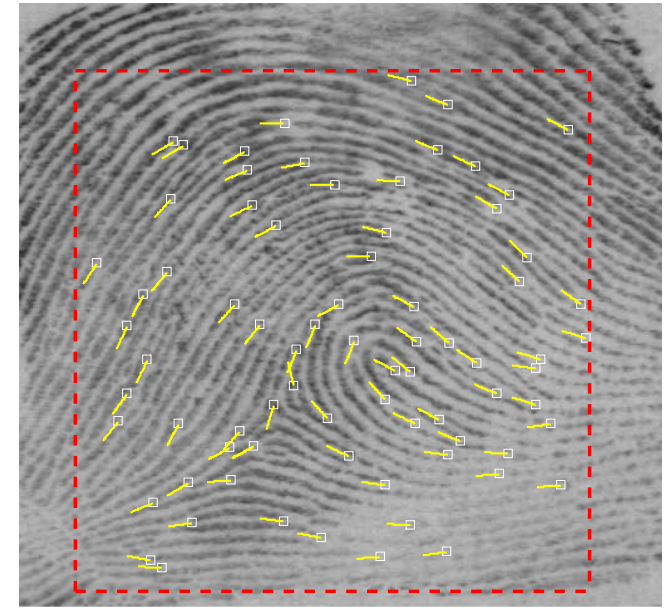

(c)

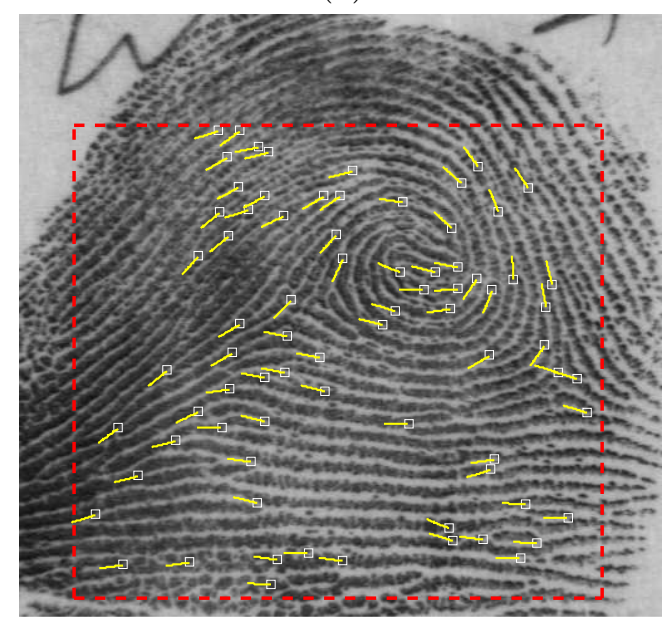

(e)

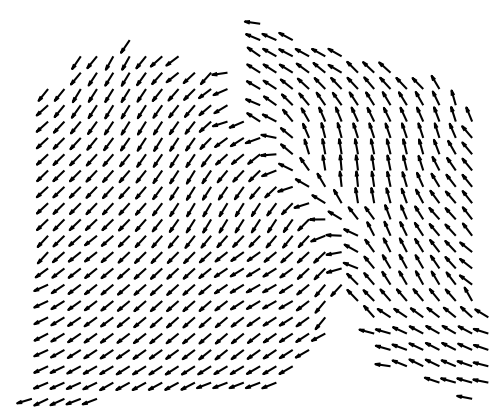

(b)

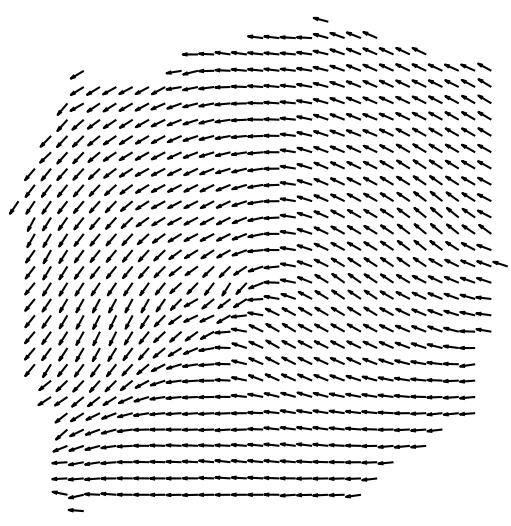

(d)

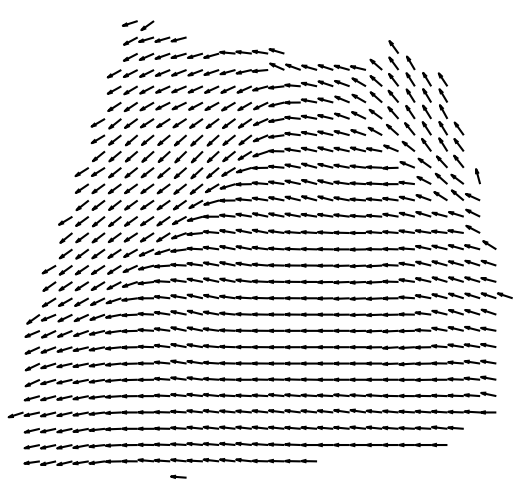

(f)

Figure 2.10: (a), (c) and (e) are original fingerprints of L, R and W whereas (b), (d) and (f) are their estimated orientation maps. 
2. Compute Sobel gradients $G_{x}$ and $G_{y}$ at each pixel in a block and assign the average of these gradient values to the corresponding grid point. The Sobel masks are given by,

$$
G_{x}=\left(\begin{array}{ccc}
1 & 2 & 1 \\
0 & 0 & 0 \\
-1 & -2 & -1
\end{array}\right), G_{y}=\left(\begin{array}{ccc}
-1 & 0 & 1 \\
-2 & 0 & 2 \\
-1 & 0 & 1
\end{array}\right) \text {. }
$$

3. The orientation for a grid point is computed as,

$$
\theta(i, j)=\frac{1}{2} \arctan \left(\frac{V_{y}(i, j)}{V_{x}(i, j)}\right)
$$

where,

$$
V_{x}(i, j)=\sum_{u=i}^{i+w} \sum_{v=j}^{j+w} 2 G_{x}(u, v) G_{y}(u, v), V_{y}(i, j)=\sum_{u=i}^{i+w} \sum_{v=j}^{j+w} G_{x}^{2}(u, v)-G_{y}^{2}(u, v) .
$$

The estimated and true orientation maps, $(\boldsymbol{\theta}, \hat{\boldsymbol{\theta}})$, for different fingerprints in the NIST-4 database are shown in Figure 2.11.

To determine the similarity between the true and estimated orientation maps, we compute the correlation coefficient, $r(\theta, \hat{\boldsymbol{\theta}})$, given by, $r(\theta, \hat{\boldsymbol{\theta}})=\frac{\sum_{m} \sum_{n}(\theta-\bar{\theta})(\hat{\boldsymbol{\theta}}-\overline{\hat{\boldsymbol{\theta}}})}{\sqrt{\left(\sum_{m} \sum_{n}(\boldsymbol{\theta}-\overline{\boldsymbol{\theta}})^{2}\right)\left(\sum_{m} \sum_{n}(\hat{\boldsymbol{\theta}}-\overline{\hat{\boldsymbol{\theta}}})^{2}\right)}}, 0 \leq|r| \leq$ 1 , where, $m \times n$ is size of the orientation maps, $\overline{\boldsymbol{\theta}}$ and $\hat{\hat{\boldsymbol{\theta}}}$ represent the mean of $\boldsymbol{\theta}$ and $\hat{\boldsymbol{\theta}}$, respectively. Figure 2.12 that plots the histogram of correlation coefficients as observed on the NIST-4 database, validates our technique and indicates that the estimated ridge orientations are consistent with the underlying (true) ridge flow. Due to the absence of 'valid' triplets around the core region, the estimated orientation map can not capture these regions, e.g., the recurving back in loops or the concentric pattern in whorls.

To improve the performance of fingerprint matching, the estimated orientation map may also be used along with the minutiae distribution. The orientation estimation algorithm can be beneficial in applications like smart cards (where memory is critical), since the orientation field need not be stored explicitly but can be generated from the template itself. 

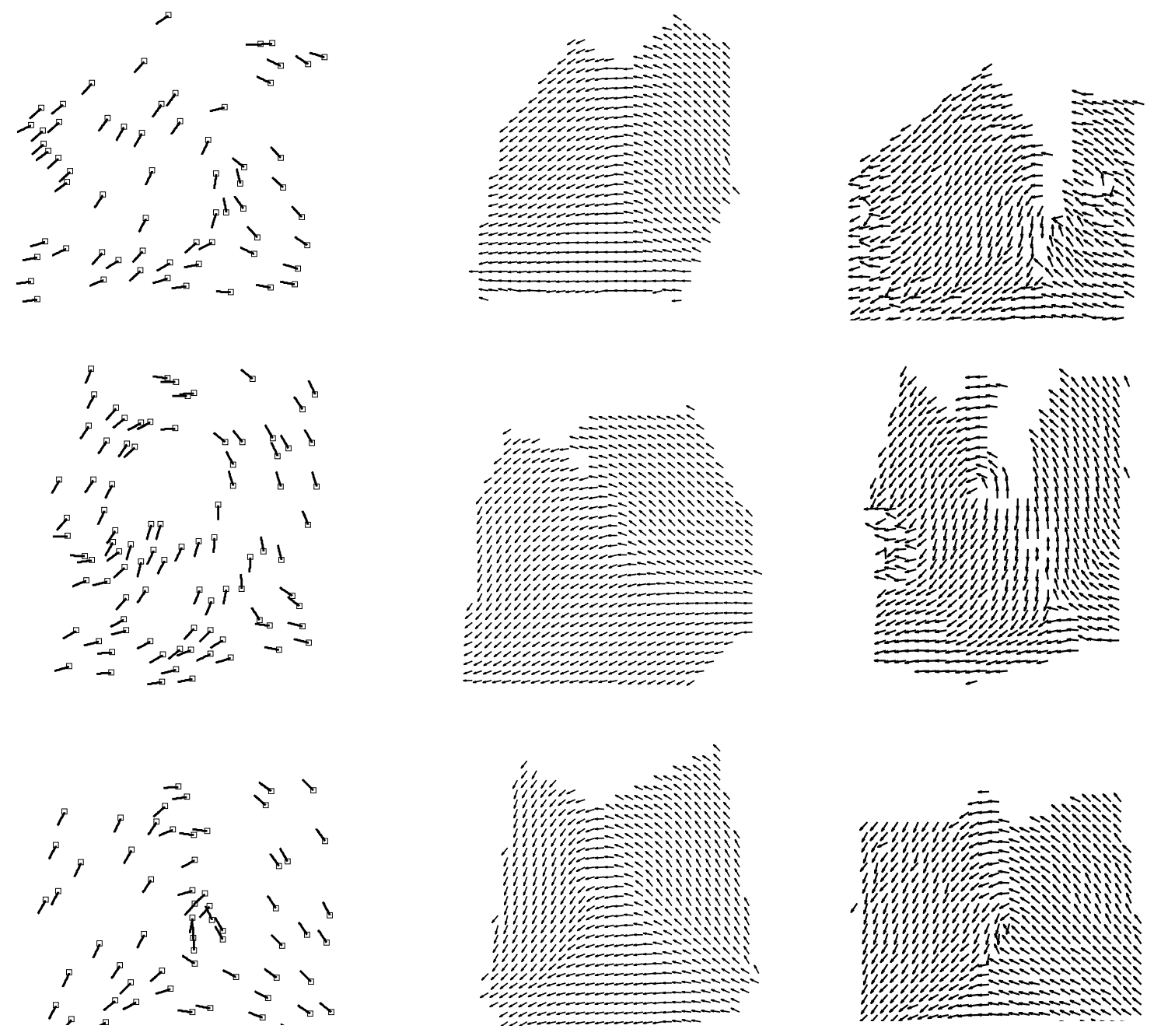

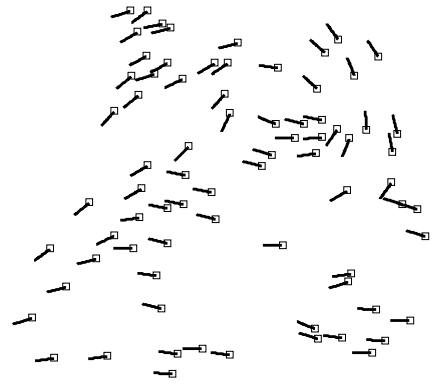

(a)

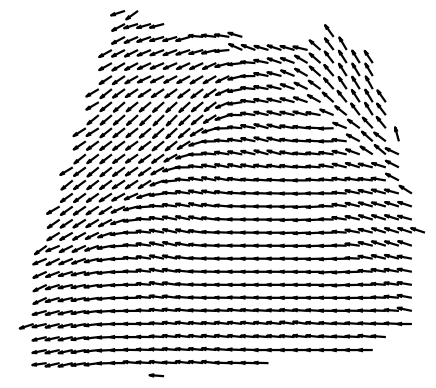

(b)

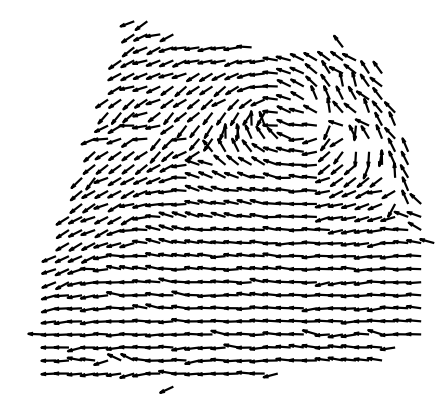

(c)

Figure 2.11: Comparing the estimated orientation map (from minutiae) with the true map: (a) Minutiae plot of a fingerprint, (b) Estimated orientation map, (c) True orientation map. Due to absence of valid triplets around the core region, the estimated orientation map cannot capture these regions, e.g., the concentric pattern in whorls. 


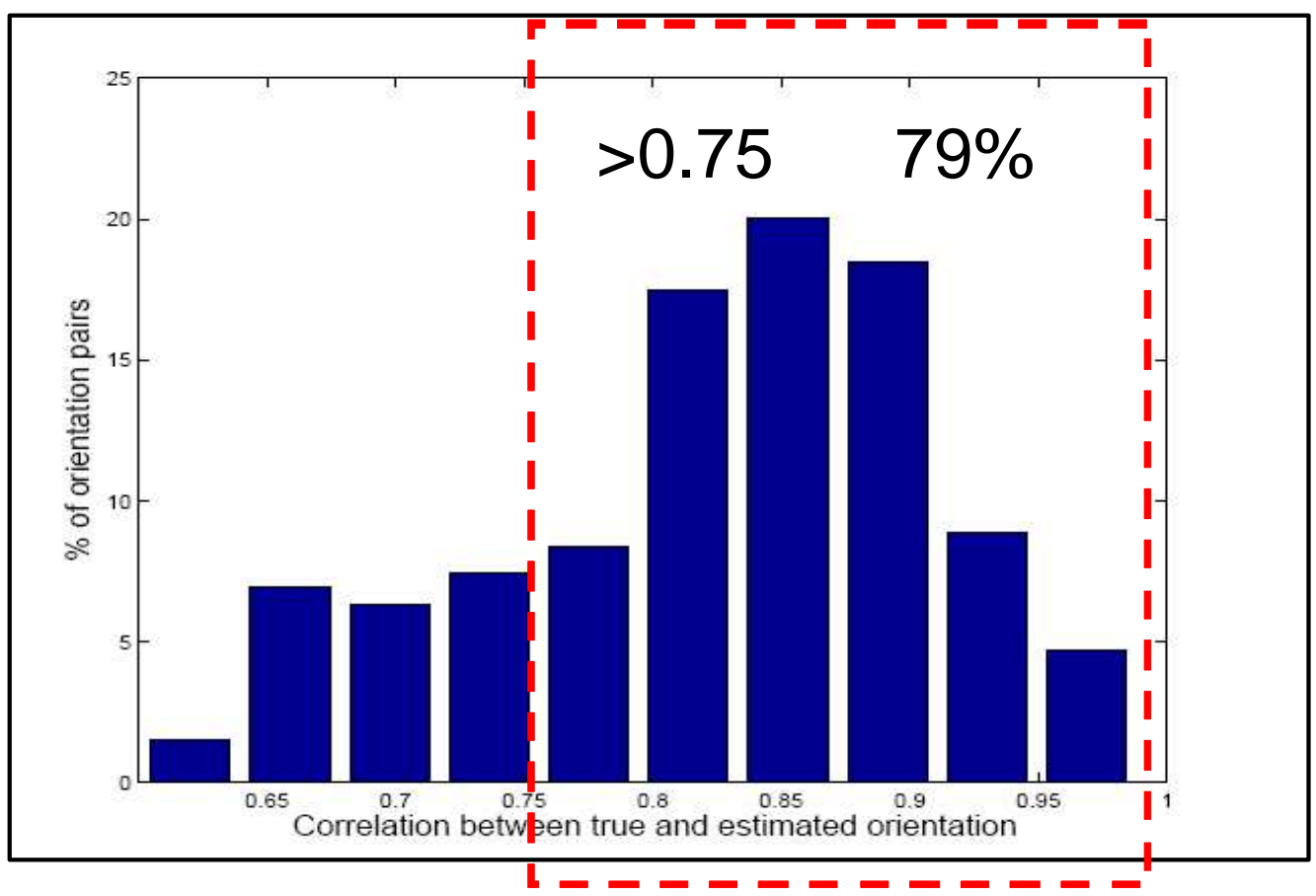

Figure 2.12: Correlation between $\hat{\boldsymbol{\theta}}$ and $\theta$ as observed on the NIST 4 database. About $79 \%$ of the orientation pairs have correlation more than 0.75 . 


\section{Chapter 3}

\section{Fingerprint Classification Using Minutiae Points}

\subsection{Fingerprint classification}

The ridge flow in the fingerprints constitute a pattern which can be systematically categorized into a fixed number of non-overlapping classes. Fingerprint classification refers to the problem of assigning a fingerprint to a class in a consistent and reliable way [4]. This is an important property of fingerprints since it helps in reducing the search time in identification schemes. The Galton-Henry [2] classification scheme partitions fingerprints into five classes, namely, arch (A), tented $\operatorname{arch}(\mathbf{T})$, right loop $(\mathbf{R})$, left loop $(\mathbf{L})$ and whorl $(\mathbf{W})$ as shown in Figure 3.1. We treat this as a four-class problem by combining fingerprints of $\mathbf{T}$ and $\mathbf{A}$ into a single class (A). The number

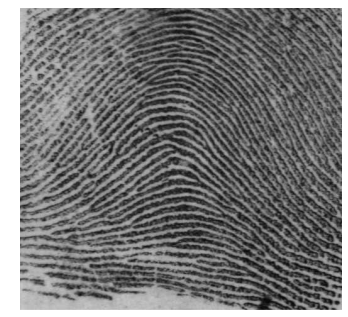

(a) Arch

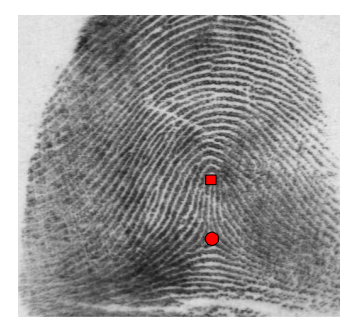

(b) Tented Arch

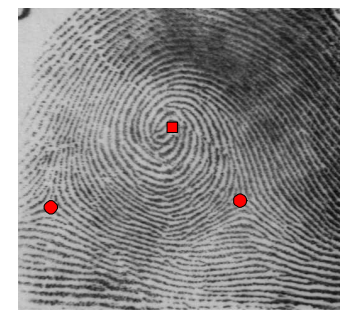

(c) Whorl

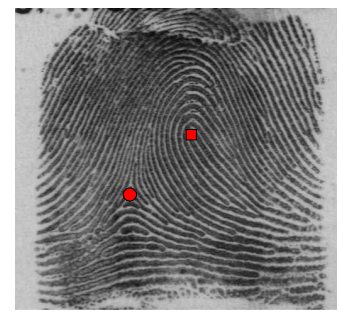

(d) Right Loop

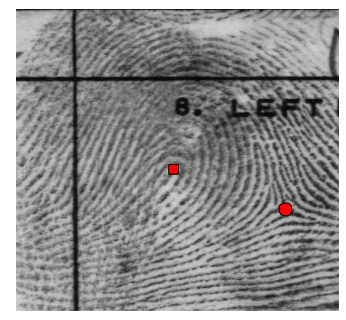

(e) Left Loop

Figure 3.1: Five classes of fingerprints according to Galton-Henry classification scheme [2]. Core is represented by a square whereas the delta by a circle.

of singularities (core and delta points) and the relative positions vary across the 5 fingerprint classes as summarized in table 3.1. Characteristics of each of these classes are described below: 


\begin{tabular}{|c|c|}
\hline Fingerprint class & Number and position of singularities \\
\hline Arch & No singularities \\
\hline Tented Arch & One core and one delta, vertically aligned \\
\hline Leftloop & One core and one delta, delta on right side \\
\hline Rightloop & One core and one delta, delta on left side \\
\hline Whorl & One core and two deltas, one delta on each side \\
\hline
\end{tabular}

Table 3.1: Number and position of singularities in five fingerprint classes.

1. Arch: This is the simplest fingerprint class since it does not have any singularities (core or delta). Ridges enter from one side of the finger and exit from the other forming a shape of an arch.

2. Tented Arch: These are similar to (plain) arch, except that it has at least one ridge which has an up thrust. It has one core and one delta which are vertically aligned.

3. Whorl: The most complex form of fingerprints is the whorl pattern. It has one core, two delta points, and a whorl-like pattern near the core. The whorl pattern is characterized by at least one ridge traversing a 360 degree closed path around the core.

4. Left Loop: It is characterized by one core and one delta point. One or more ridges enter from left of the finger and exit from the same side, thus forming a loop. It has a delta where the ridges converge or diverge into two branches on the right side.

5. Right loop: Like left loop, it has one core and one delta. One or more ridges enter from right side of the finger and exit from the same side by forming a loop. It has a delta on the left side.

\subsubsection{Need for fingerprint classification}

Generally, the database of a fingerprint authentication system includes millions of fingerprints. Large volumes of fingerprints are collected and stored every day in a wide range of applications, including access control (e.g. ATM), law forensics (e.g. FBI database contains more than 200 million fingerprints [4]), driver's license registration of state, etc.

For the identification, the simplest technique is to scan the entire database in order to retrieve the top matches. This brute force method is obviously an inefficient and impractical way to 
address the problem due to a very large response time. To alleviate this problem, fingerprints are partitioned into predefined classes and are stored in separate bins in the database. At the time of identification, the class of the query fingerprint is determined and it is compared against the fingerprints of this class alone.

\subsubsection{Various approaches for classification}

Automated fingerprint classification is a difficult pattern recognition problem. In spite of substantial research in this field, it is still an active field of research. A detailed discussion about various methods proposed for fingerprint classification is available in [4]. Most existing approaches use the following features along with the fingerprint image itself:

1. Orientation image : Most existing classification techniques make use of the orientation image. If reliably computed, it is alone sufficient for classification. Cappelli and Maltoni proposed a technique in which the orientation image is divided into homogeneous regions using a cost function [27]. A prototype model is formed for each class using these regions. A fingerprint is classified by comparing the relational graphs with the class prototypes. This technique is rotation and translational invariant and does not involve singularities.

2. Singularities : In this technique, singularities such as core and delta are detected. The fingerprints are classified based on the number and locations of these singularities [4]. Although it is relatively a simple technique, the performance is highly dependent on the accuracy of core/delta detection. In case of noisy fingerprints, singularity detection is not only difficult but can be misleading. Due to the small fingerprint area, dab prints generally do not contain deltas.

3. Ridge flow : Ridge line flow is nothing but a set of curves running parallel to ridge lines. These curves do not necessarily coincide with the fingerprint ridges and vallyes, but they exhibit the same local orientation. It can be traced by drawing lines curves locally oriented to the orientation image [28]. Jain and Minut [3] form predefined kernels corresponding to each class using this ridge flow. A fingerprint is assigned a class based on fitting it to one of these kernels. The best fitting kernel decides the class of the fingerprint.

4. Gabor filter responses : Jain et.al [29] proposed a classification algorithm using a bank of Gabor filters. The algorithm separates the number of ridges present in four directions 
$\left(0^{\circ}, 45^{\circ}, 90^{\circ}, 135^{\circ}\right)$ by filtering the central part of a fingerprint with the bank of Gabor filters. This information is quantized to generate a 'FingerCode' which is used for classification.

Some classification schemes proposed in literature use more than one feature. For example, Candela et al. [28] developed PCASYS (Pattern-level Classification Automation SYStem). It is a probabilistic neural network that is based on features derived from orientation image. It also contains a ridge tracing module, which traces the ridge flow in the bottom part of the whorl type fingerprint.

Various features that have been used so far for fingerprint classification are summarized in Table 3.2 .

\begin{tabular}{|l|c|}
\hline Authors & Features used \\
\hline Candela et al. 1995 [28], Hong et al. 1999 [30], Chong et al. 1997 [31] & Ridge line flow \\
\hline Cappelli et al. 1999 [27], Senior 2001 [32], Wilson et al. [33] & Orientation image \\
\hline Karu and Jain 1996 [34], Cho et al. 2000 [35] & Singularities \\
\hline Jain et al. [29], Yao et al. 2001 [36] & Gabor filters \\
\hline Ross et al. [37] & Minutiae points \\
\hline
\end{tabular}

Table 3.2: A summary of different fingerprint classification techniques.

\subsection{Novel use of minutiae for classification}

To best of our knowledge, none of the existing classification schemes use minutiae properties for classification. Typically, minutiae points have been used only for fingerprint alignment and matching. We propose a novel idea of using minutiae points alone for classifying fingerprints (without accessing images).

It is observed in the forensic literature that the minutiae distribution varies from class to class [38, 39, 8]. The minutiae plots of all four classes are shown in Figure 3.2. A visual glance at these minutiae plots suggests the possibility of deducing the fingerprint class from the minutiae points. Our hypothesis is based on observations made by forensic experts in the context of fingerprint individuality models. Fingerprint minutiae have been the central focus in the study of fingerprint individuality. Stoney describes 10 different individuality models proposed by various forensic experts. All these models are based on minutiae properties [8]. Most of the individuality models are based on heuristic observations and on a limited database. We have demonstrated the results over larger fingerprint database. 


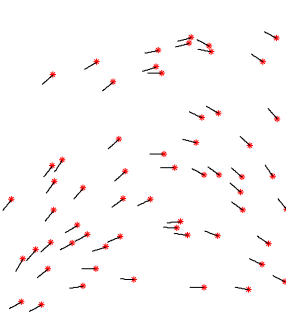

(a)

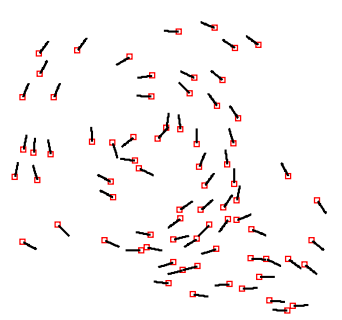

(b)

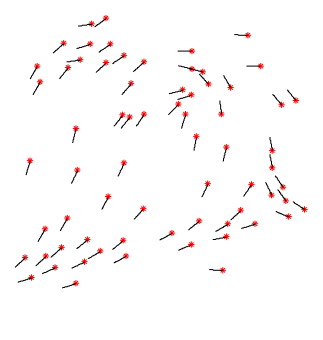

(c)

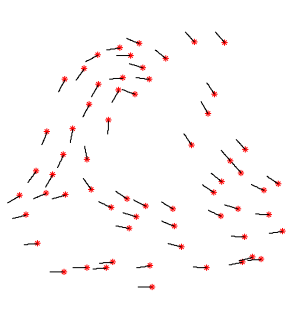

(d)

Figure 3.2: Minutiae plots of 4 fingerprint classes: (a) A, (b) W, (c) L, and (d) R.

As mentioned earlier, we assume that the stored minutiae template does not give information about the shape of the fingerprint directly. We studied the relation between minutiae properties and class of the fingerprints of NIST 4 database. Our observations and the findings listed by various forensic experts support our hypothesis that the seemingly random distribution of minutiae in a fingerprint can reveal important information about the class of a fingerprint. Most forensic experts have invested their efforts in studying fingerprints visually. Our goal is to imitate the performance of a human fingerprint expert for classification of fingerprint using minutiae template without access to the fingerprint image itself.

Most commercially available fingerprint authentication systems store minutiae templates which are later used for matching. We claim that with the proposed classifier, these templates can be also used for fingerprint classification. This eliminates the need for existing complex image processing techniques on fingerprint images for classification. We assume that the simplest form of minutiae template $(x, y, \theta)$ is available and the information about the singularity points, class etc is unknown. (The basic shape of the fingerprint is decided by location and orientation of singularities. If the minutiae template were to store the position and orientation of singularities then determining the class of fingerprint is a trivial task.)

\subsubsection{Minutiae based classification algorithm}

Predicting the class of a fingerprint from its minutiae points alone is not a easy task. This is due to the fact that the fingerprint can be enrolled in any orientation or translation. So the individual minutiae points can literally be in any position and orientation for the same fingerprint. Thus, the features we have chosen for fingerprint classification are translation and 
rotation invariant. The main steps of our minutiae-based classification algorithm are as described in the block diagram shown in Figure 3.3. We have described the orientation estimation scheme

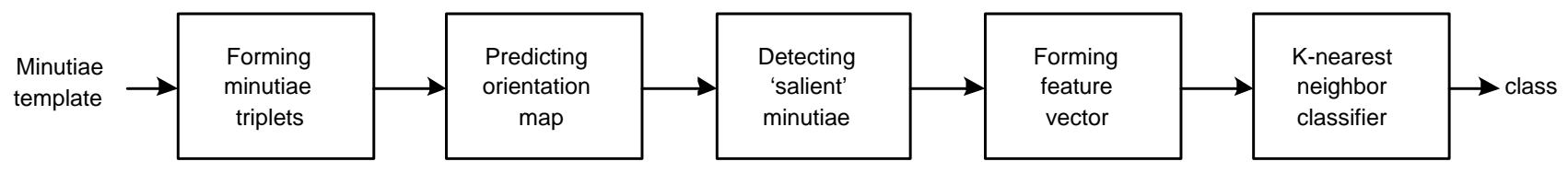

Figure 3.3: Block diagram showing main steps of minutiae-based classification algorithm.

using minutiae triplets in Chapter 2 .

1. Detecting salient minutiae : As mentioned above, the designed classifier is based on minutiae information and the estimated orientation of the fingerprint to be classified. A fingerprint can be divided into 3 parts namely base, core and marginal areas. By visually analyzing fingerprint ridge patterns across classes, it is clear that they have almost the same ridge structure in the base and marginal area as shown in Figure 3.4. But the irregularities in the vicinity of the core area are significant for classification, such as a circular ridge pattern in case of whorls or curving back of ridges in loops. Accordingly, the minutiae

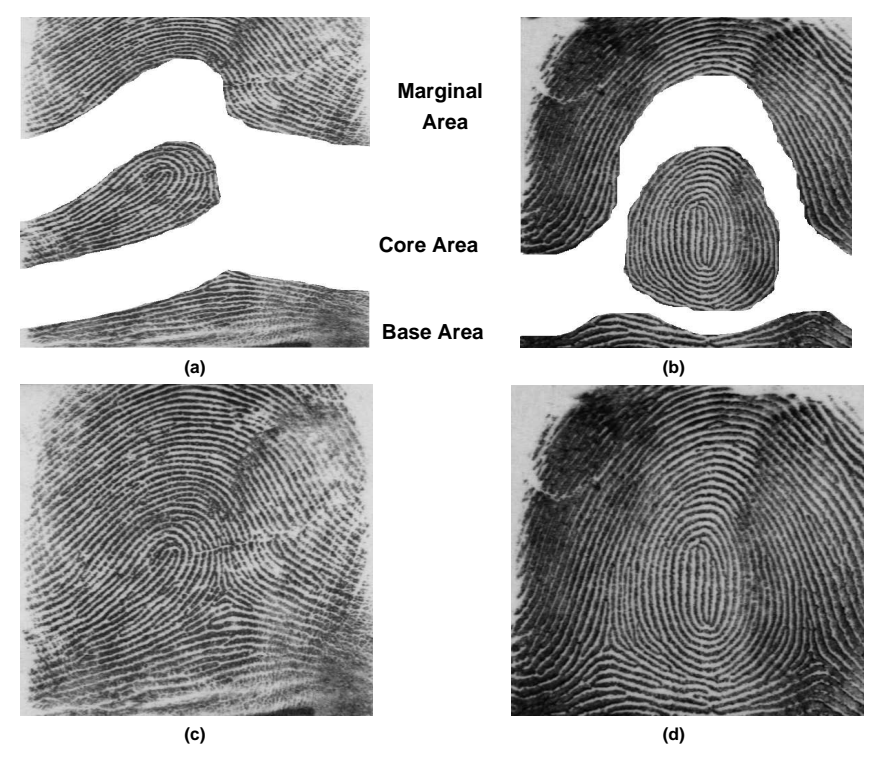

Figure 3.4: (a) and (b) show $\mathbf{L}$ and $\mathbf{W}$ fingerprints divided into various areas whereas (c) and (d) show the corresponding original fingerprints respectively.

present in the vicinity of the core point have important class representative characteristics. We refer to these as 'salient' minutiae and use them for classification. The ridges around 
the core point have high curvature and form a nearly circular pattern across all classes as shown in Figure 3.2. In order to select the salient minutiae, we first detect a registration point $\left(R_{0}\right)$ using Hough transform (The salient minutiae reside around $R_{0}$ ). Our motivation behind using Hough transform is the inherent circular-like ridge structure of fingerprints in the vicinity of core region.

The Hough transform is a technique which can be used to isolate features of a particular shape within an image. The classical Hough transform is most commonly used for the detection of regular curves such as lines, circles, ellipses, etc [40]. It is particularly useful for computing a global description given the local (noisy) measurements. In our case, the local information consists of minutiae coordinates and their orientations. The parametric equation of a circle is

$$
\left(x-x_{0}\right)^{2}+\left(y-y_{0}\right)^{2}=r^{2},
$$

where ' $x_{0}$ ' and ' $y_{0}$ ' are the coordinates of the center of the circle and ' $r$ ' is the radius. The parameter space consists of three coordinates viz. the position of minutiae point $(x, y)$ and its orientation $\theta$.

As mentioned above, the orientation of minutiae in the vicinity of the core region define a circle. Consider a circle formed by minutiae as shown in Figure 3.5. Let ' $\mathrm{L}$ ' be a perpendicular line to a minutiae orientation. This line passes through the center of the circle. Our goal here is to detect the center. It can be observed that these minutiae have

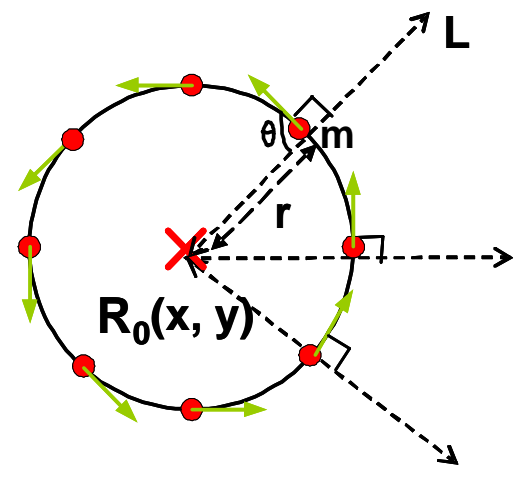

(a)

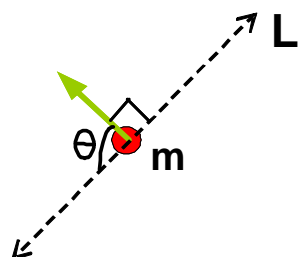

(b)

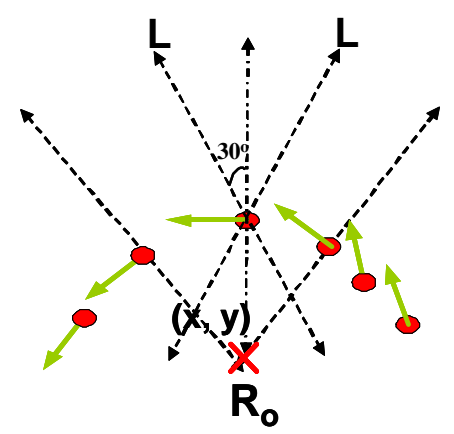

(c)

Figure 3.5: (a) Circular plot of minutiae, (b) Line ' $\mathrm{L}$ ' is perpendicular to the orientation of minutiae $\mathrm{m},(\mathrm{c}) \mathrm{L}$ made nearly perpendicular $\left( \pm 30^{\circ}\right)$ to the orientation $\theta$. 
each minutiae ' $\mathrm{m}$ ' we traverse its line ' $\mathrm{L}$ ' perpendicular to the orientation of minutiae $\theta$ at discrete points as illustrated in Figure 3.5 (b). These points $(x, y)$ correspond to the probable center points of the circle. The radius ' $\mathrm{r}$ ' of each of these circles is nothing but distance from minutiae to the corresponding center points as the minutiae ' $\mathrm{m}$ ' lies on their circumferences. A 3D accumulator in Hough space corresponding to the center $(x, y)$ and radius $r$ is used. Given a minutiae template $\mathrm{M}=m_{i}, i=1$ to $N$, the algorithm for detecting the $R_{0}$ is as follows.

(a) Initialize the accumulator $A(x, y, r)$ where $(x, y)$ is the center of the potential circle with radius $r$.

(b) For each minutiae $m_{i}, i=1$ to $N$, the potential centers $(x, y)$ lie on the line ' $\mathrm{L}$ '. The accumulator cell $A(x, y, r)$ is incremented if the point $(x, y)$ is at distance $r$ from minutiae $m_{i}$.

(c) For a circular minutiae pattern, there will be a well-pronounced peak in the Hough parameter space for one center $(x, y)$ (point marked with red '*' in Figure 3.5 (a)). This corresponds to the registration point which is characterized by significant minutiae activity around it.

We observed that rather than using only the minutiae information, if we use the estimated orientation map then the performance of Hough transform is improved. As mentioned earlier, orientation map is a discrete matrix. Each cell of the orientation map represents the average estimated orientation for that cell. We perform above procedure for all the starting coordinates of the non-empty cells of the orientation map. Note that the ridge structure in fingerprints is not exactly circular thus in our experiments, we use lines nearly perpendicular $\left( \pm 30^{\circ}\right)$ to the orientation $\theta$ as shown in Figure $3.5(\mathrm{c})$. The results for Hough transform using the estimated orientation maps are as shown in Figure 3.6. Note that $R_{0}$ is mostly detected in the vicinity of the true core. Once $R_{0}$ is detected, the minutiae located in a $300 \times 300$ region about $R_{0}$, are selected for classification as shown in Figure 3.7.

2. Generating feature vectors : Each fingerprint is represented by a feature vector, set of characteristic measurements extracted from salient minutiae. These features capture vital properties of minutiae such as their relation with neighboring minutiae, relation between 


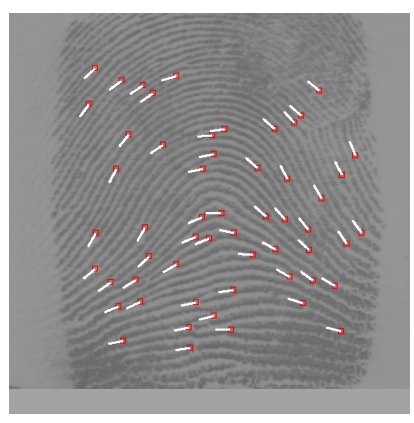

(a)

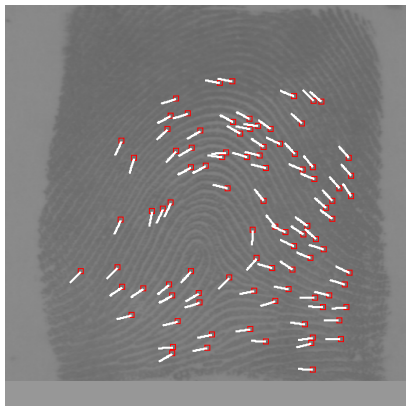

(c)

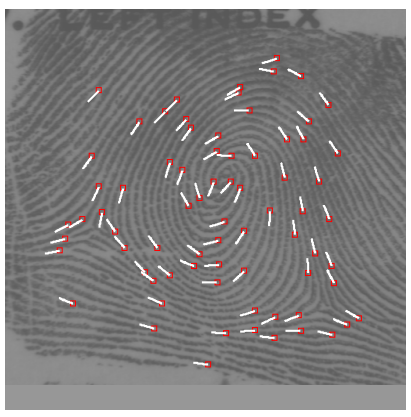

(e)

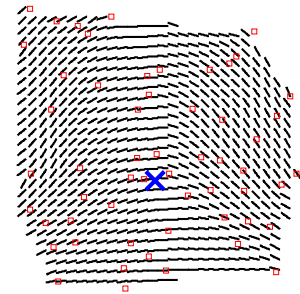

(b)

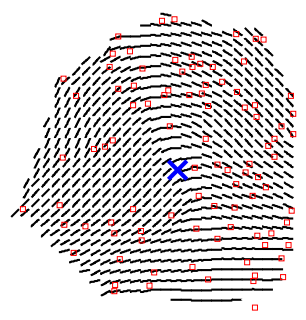

(d)

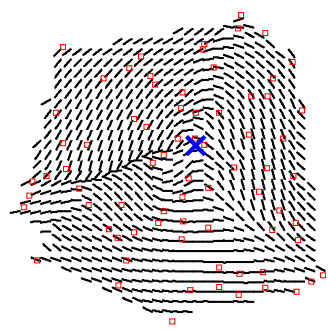

(f)

Figure 3.6: Minutiae plot overlaid on original fingerprint of $\mathbf{A}, \mathbf{L}$ and $\mathbf{W}$ are shown in (a), (c) and (e) respectively. The corresponding $R_{0}$ points marked in blue ('X') detected using estimated orientation maps are shown in (b), (d) and (f). 


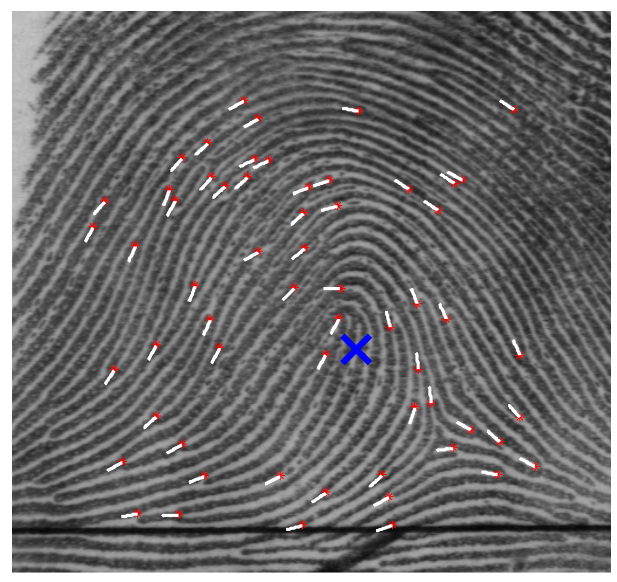

(a)

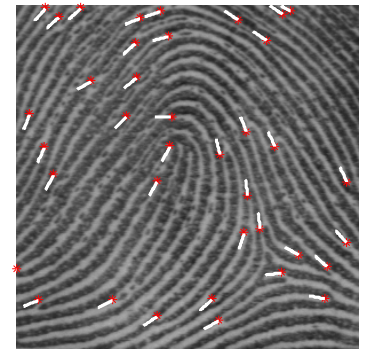

(b)

Figure 3.7: (a) The orientation map and detected registration point marked in blue ('*'), (b) Salient minutiae in a $300 \times 300$ frame about $R_{0}$.

their coordinates and orientations, their clustering tendencies, etc. Most of these classrepresentative minutiae properties were studied by various forensic experts for proposing individuality models and for understanding the unique nature of fingerprints but not for classifying the fingerprints [39, 8, 38, 26]. The features we have used are invariant to rotation, translation and scaling of fingerprint images. The 11-dimensional vector $F=$ $\left\{F_{1}, F_{2}, \cdots, F_{11}\right\}$ is constructed as follows.

(a) Features based on minutiae orientations $\left(F_{1}, F_{2}\right)$ : Stoney claims that minutiae orientations are robust to fingerprint distortions. The direction of ridge flow varies from one class to other and so do minutiae orientations. For instance, $\mathbf{W}$ has at least one ridge which makes 360 degree closed path in the central region of the fingerprint. Thus the orientations of these minutiae range from $0-360$ degrees. On the contrary, the minutiae orientations of $\mathbf{A}$ have only two dominant directions. Roxburgh [38] observed correlation between minutiae orientations and class of the fingerprint. In order to understand the distribution of minutiae orientations for each class, we examined the rose plots ${ }^{1}$ of minutiae orientations (Figure 3.8). It can be noted that the rose plots for whorl and arches are different. Whorl is characterized by high minutiae density around the core region. Also due to the circular pattern, the minutiae orientations are in various directions. So the rose plot for $\mathbf{W}$ is distributed in all four quadrants of the

\footnotetext{
1 The rose plot is a a polar plot showing the histogram of angles.
} 
rose plot. Arches have comparatively less number of minutiae. Also their orientations fall in fixed quadrants, hence the rose plot has two dominant peaks. The rose plots for $\mathbf{L}$ and $\mathbf{R}$ are mirror images of each other. One feature capturing these variations in minutiae orientations across classes is the number of empty bins in the rose plot $\left(F_{1}\right)$. This indirectly captures the spread of minutiae orientations. The feature $F_{2}$ is the variance in minutiae orientations of a minutiae template.

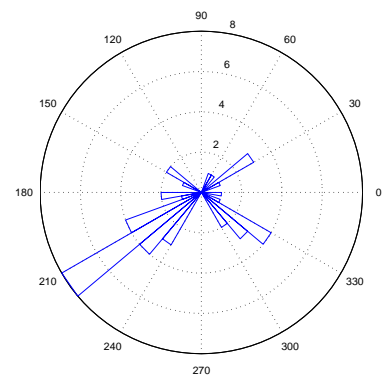

(a)

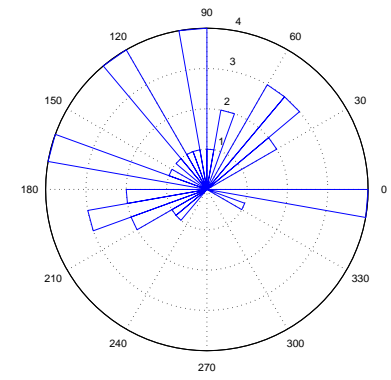

(b)

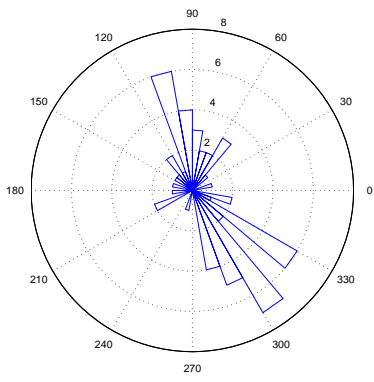

(c)

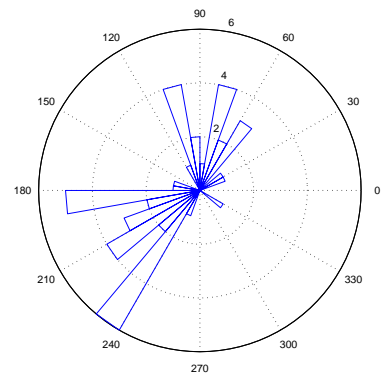

(d)

Figure 3.8: Rose plots of (a) A, (b) W, (c) L, (d) R.

(b) Features relating minutiae pairs $\left(F_{3}, \cdots, F_{6}\right)$ : The fingerprint individuality model proposed by Stoney and Thornton uses minutiae pairs [8]. They suggest that minutiae pairs are a fundamental unit for representing variations in fingerprints . They also found a strong correlation between the spacing and orientations of minutiae pairs. Also Roxburgh [38] considered correlation between neighboring minutiae and variation in minutiae position relative to the pattern of fingerprint for proposing his individuality model. The properties of minutiae in a local neighborhood vary across classes. For instance, the neighboring minutiae in the central region of $\mathbf{W}$ have large orientation difference where as minutiae neighbors in $\mathbf{A}$ have similar orientations. The correlation between spatial location and orientations of minutiae pairs can be examined by estimating the joint distribution of ' $R$ ' and ' $\Phi$ ' where $R$ is the distance between two minutiae and $\Phi$ is the orientation difference as shown in equations (3.2) to (3.5). The first distribution corresponds to minutiae pairs which are spatially close to each other and having almost similar orientations, and second to the pairs which are close but having large orientation difference. Similarly, the third joint distribution includes minutiae pairs away from each other and having similar orientations whereas fourth includes pairs away from each other but having large orientation differences. Figure 
3.9 shows all the four types of minutiae pairs.

$$
\begin{gathered}
F_{3}=\sum_{0 \leq R \leq R_{1}} \sum_{0 \leq \Phi \leq \Phi_{1}} P(R, \Phi) d R d \Phi, \\
F_{4}=\sum_{0 \leq R \leq R_{1}} \sum_{\Phi_{1} \leq \Phi \leq \Phi_{2}} P(R, \Phi) d R d \Phi, \\
F_{5}=\sum_{R \geq R_{2}} \sum_{0 \leq \Phi \leq \Phi_{1}} P(R, \Phi) d R d \Phi, \\
F_{6}=\sum_{R \geq R_{2}} \sum_{\Phi_{1} \leq \Phi \leq \Phi_{2}} P(R, \Phi) d R d \Phi,
\end{gathered}
$$

In our experiments, we have taken $R_{1}=60$ pixels, $R_{2}=180$ pixels, $\Phi_{1}=30^{\circ}$ and $\Phi_{2}=180^{\circ}$. It is observed that these distributions are significantly different for all 4 classes. The above probabilities can be computed by finding the number of pairs satisfying the above mentioned criteria for $R$ and $\Phi$ divided by the total number of minutiae pairs formed in a template. For $\mathbf{A}, F_{3}$ is larger among four features whereas for whorls, $F_{4} 3.9$ We observed that for loops, the values for $F_{1}, F_{3}$ are larger.

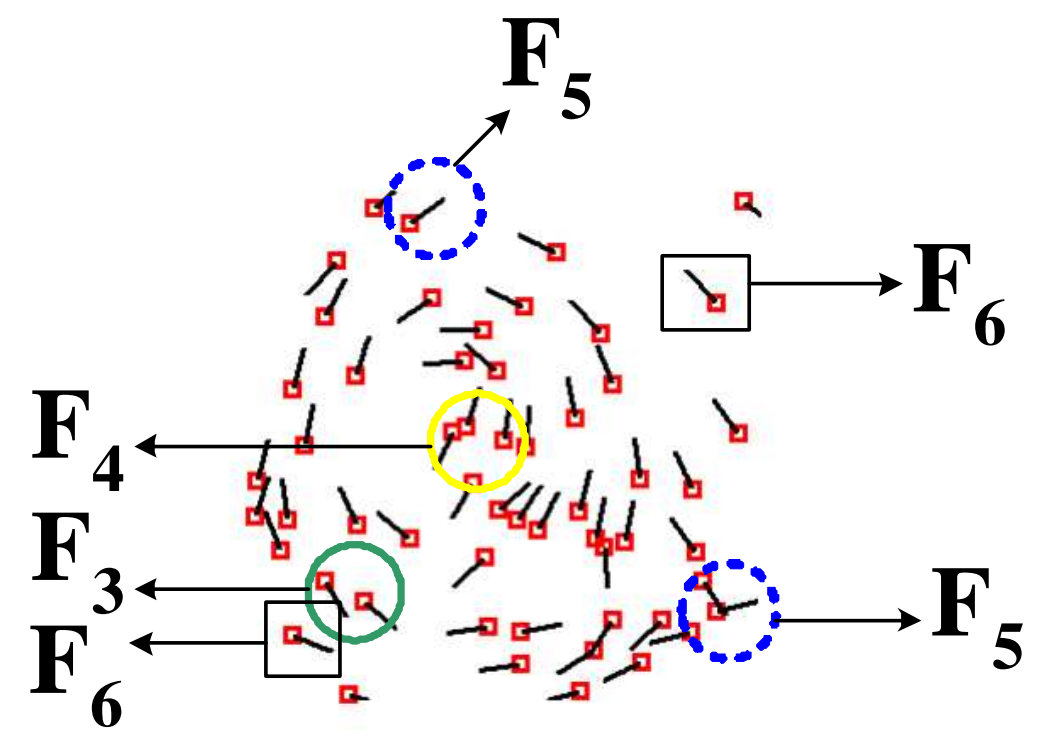

Figure 3.9: A whorl minutiae plot showing 4 types of minutiae pairs.

(c) Features representing clustering of minutiae $\left(F_{7}, F_{8}\right)$ : It is observed that minutiae tend to cluster. Kingston [26] estimated probability of a particular number 
of minutiae from the minutiae density assuming Poisson distribution. He observed variations in minutiae density among different-sized regions across different locations within the fingerprint (minutiae density increases near core and delta regions). Stoney, Champod and Margot observed that there are variations in minutiae densities due to different patterns of the ridge flow, for example, in the region of recurving ridges [8]. If an equal number of ridges flow in and out of a region, then for each minutia that produces a ridge, there must be a minutia which consumes a ridge. An imbalance in number of minutiae orientations results in converging or diverging ridges. There exists a fundamental relationship between minutiae and ridge pattern. Also Galton [39] showed that there is a strong correlation between the class of a fingerprint and the occurrence of a minutiae at a specific location in the image (Figure 3.10). Whorls are characterized by high minutiae density near the core region. The clusters of minutiae are obtained in various regions of fingerprint. Champod and Margot observed that these regions vary from class to class [41]. $F_{7}$ is the maximum minutiae density obtained in each fingerprint. This value is relatively maximum for $\mathbf{W}$ and least for $\mathbf{A}$. We sample the local minutiae in a radius of 50 pixels to compute the minutiae density. The feature $F_{8}$ is the maximum variance in minutiae orientations in a minutiae cluster within this radius.

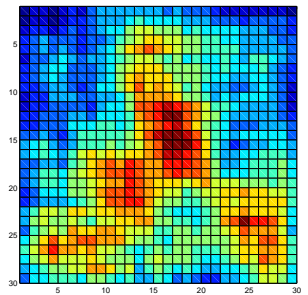

(a)

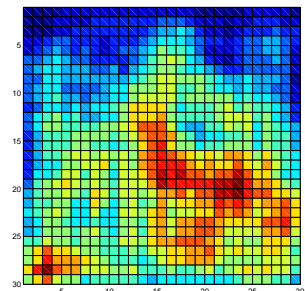

(b)

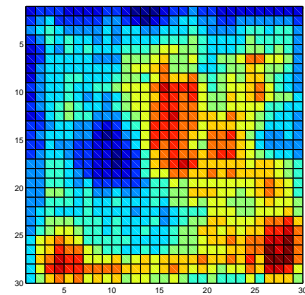

(c)

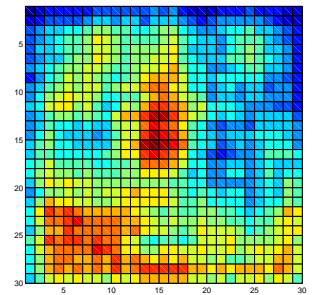

(d)

Figure 3.10: Minutiae density associated with 4 different classes of fingerprints: (a) A, (b) W , (c) $\mathbf{L}$, and (d) $\mathbf{R}$. These plots were generated using 30 images per class. Blue indicates a low density region while red indicates a high density region.

(d) Features capturing global ridge information $\left(F_{9}, F_{10}, F_{11}\right)$ : Visually (Figure $2.2)$ it is apparent that features $F_{1} \cdots F_{8}$ can distinguish classes $\mathbf{A}$ and $\mathbf{W}$ but are not sufficient for reliably resolving ambiguity between the classes $(\mathbf{L}, \mathbf{R}),(\mathbf{A}, \mathbf{L}, \mathbf{R})$ and $(\mathbf{L}, \mathbf{R}, \mathbf{W})$. It is necessary to include information about global ridge pattern 
along with the local minutiae properties. To capture the global ridge structure of the fingerprint, we have defined geometric kernels which model the shape of the fingerprint around the core region for $\mathbf{W}$, Land $\mathbf{R}$ classes. In a left loop, the ridges in the core region form a loop by curving back to the left side of the fingerprint. This is captured by a kernel formed using two semi-ellipses corresponding to the concave and convex parts of the loop. The kernel for $\mathbf{R}$ is nothing but a mirror image of this kernel. The circular ridge structure of $\mathbf{W}$ is represented using a simple circle. See Figure 3.11 .

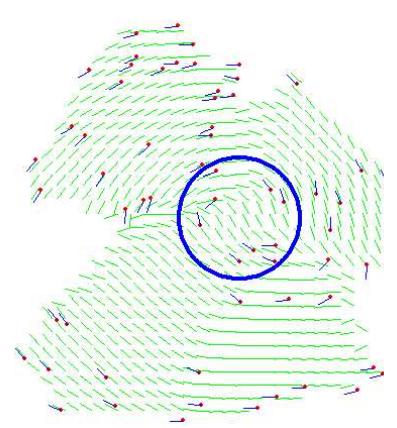

(a)

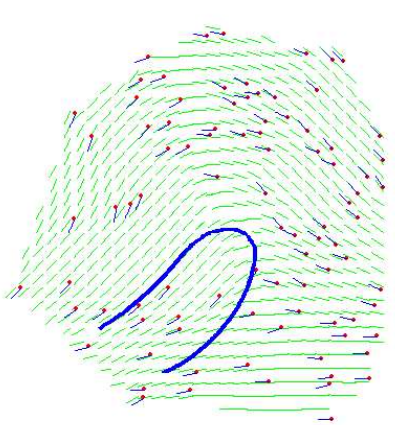

(b)

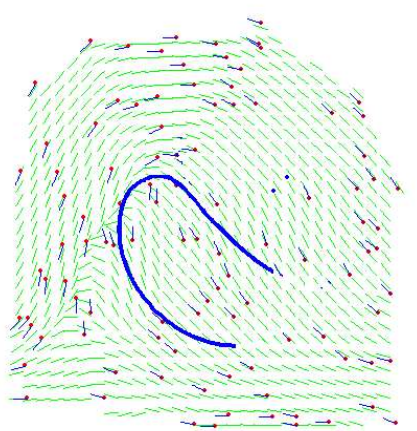

(c)

Figure 3.11: Kernel for (a) L, (b) R, and (c) W.

Above the core region of every fingerprint has arch-like characteristics (Figure 3.4). We do not define a kernel for $\mathbf{A}$ class as it would fit well for all the classes. The algorithm we have used is based on hierarchical kernel fitting approach proposed by Jain and Minut [3] for fingerprint classification with certain modifications to suit our problem. It is a model-based approach which uses only flow field of ridges for fingerprint classification. In this approach, the classification is achieved by finding the kernel that best fits to the flow field of a given fingerprint. The algorithm is presented in this paper is as follows. Consider $\mathrm{V}$ to be a smooth vector field defined over some region in the plane $R^{2}$ and let $\beta$ be its argument. Let $\gamma$ be a circular kernel curve in $R^{2}$ as shown in Figure 3.12. Let $\dot{\gamma}$ be tangent to $\gamma$ and $\alpha$ be its argument. Consider a point $\gamma_{t}=(x(t), y(t))$ present on the kernel $\gamma$. An energy functional capturing the difference between the direction of $\dot{\gamma}$ and that of the vector field $\mathrm{V}$ at point $\gamma_{t}$ is defined by

$$
E(\gamma)=\frac{\int_{\gamma} \sin ^{2}(\alpha-\beta(\gamma)) d \gamma}{\int_{\gamma} d \gamma}
$$




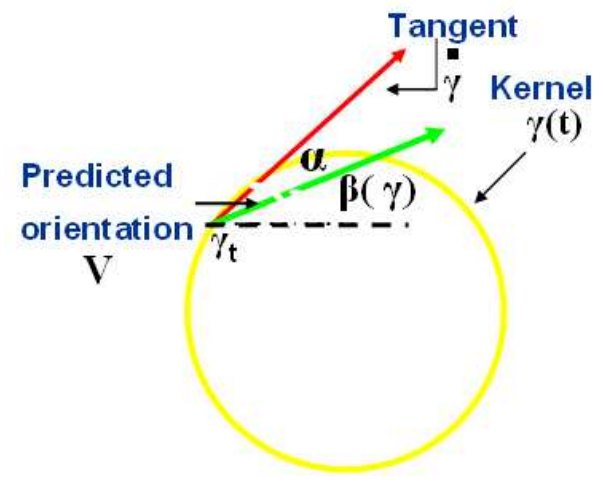

Figure 3.12: Red Vector: Unit tangent vector to the kernel, Green Vector: Direction of flow field at point $\gamma_{t}[3]$.

To find how well each kernel fits into an orientation map of the fingerprint, energy values (Equation 3.6) for various discrete points on the kernel are computed. Then the average of all the value decides the fit. Lesser average means better fitting kernel. The classification then becomes a simple energy minimization problem. This algorithm uses the original fingerprint image for finding the orientation map. In our case, we have only the partial orientation map estimated from minutiae. We use the kernel fitting approach for finding features which represent the global ridge pattern of the fingerprint. For each estimated orientation map, we find three values for energy functionals (Equation 3.6) corresponding to the $\mathbf{L}, \mathbf{R}$, and $\mathbf{W}$ kernels which form the features $\left(F_{9}, F_{10}, F_{11}\right)$ respectively. The least value represents the best fitted kernel. So for an orientation map of $\mathbf{W}$, it is expected that the minimum of three features is $F_{11}$ where as for $\mathbf{L}$ and $\mathbf{R}, F_{9}$ and $F_{10}$ respectively. We observed that due to the inherent similarity in ridge structure of loops and $\mathbf{A}$, both $\mathbf{L}$ and $\mathbf{R}$ kernels fit well for $\mathbf{A}$. So for $\mathbf{A}$ class values for $F_{9}$ and $F_{10}$ are almost the same. This property is useful for resolving ambiguity between loops and $\mathbf{A}$. These class-specific kernels are defined with respect to the $R_{0}$ obtained using Hough transform i.e. for $\mathbf{W}, R_{0}$ is the center of the circle whereas for $\mathbf{L}$ and $\mathbf{R}$, a focus of the ellipse kernel.

The rotation and translation of fingerprints are taken into account by subjecting these kernels for following transformations. In our experiments, for $\mathbf{W}$, we vary the radius of the kernel from 100 to 160 pixels. The various shapes of ellipse for $\mathbf{L}$ and $\mathbf{R}$ classes are obtained by varying the semi-major axis from 120 to 180 and semi-minor axis from 60 to 100 pixels. The angle that ellipse makes with the horizontal is varied from -10 to 
10 degrees. The kernels are moved in a window of $20 \times 20$ around $R_{0}$. To compute the features $\left(F_{9}, F_{10}, F_{11}\right)$ for a fingerprint, minimum of the energy functionals of the kernels corresponding to all these transformations is found out.

3. Classification of fingerprints : A K nearest neighbor classifier that uses the manhattan distance, is employed to classify the fingerprints. The experiment was performed on NIST4 fingerprint database which is considered as a benchmark for fingerprint classification. Typically a good quality fingerprint consists of $40-100$ minutiae [42] but this is not true for some fingerprints in NIST database. Since we need sufficient number of minutiae triplets to compute the orientation map, we reject the fingerprints having less than 25 minutiae.

We used 150 fingerprints (arbitrarily chosen) for training and 550 fingerprints for testing of each class. Each fingerprint is represented a feature vector. We observed that by reducing the dimensionality of the feature vector using exhaustive feature selection from 11 to 8 features, the classifier performance is improved. The selected 8 features are $F_{1}, F_{2}, F_{3}, F_{4}, F_{7}, F_{9}, F_{10}, F_{11}$.

We tried various values of $\mathrm{K}$ and found that the classifier performance is best for $\mathrm{K}=$ 5. The $5 N N$ classifier is applied to each feature individually. Based on the error rate, an appropriate weight is assigned for a particular feature. Thus the feature giving more error gets less weight. To find the nearest neighbor for a test fingerprint, the difference between its features with that of training fingerprint is scaled by their corresponding weight. We observe that by using this 'weighted distance', the classifier performance is further improved. We obtained a classification rate of $82 \%$ and the resultant confusion matrix is shown in table 3.3 .

\begin{tabular}{|r||c|c|c|c|}
\hline \multicolumn{1}{|c||}{ True } & \multicolumn{4}{c|}{ Assigned Class } \\
\cline { 2 - 5 } Class & A & L & R & W \\
\hline A & 467 & 45 & 32 & 6 \\
\hline $\mathbf{L}$ & 61 & 464 & 7 & 18 \\
\hline $\mathbf{R}$ & 69 & 26 & 448 & 7 \\
\hline $\mathbf{W}$ & 4 & 61 & 68 & 417 \\
\hline
\end{tabular}

Table 3.3: Confusion matrix indicating classification performance. 


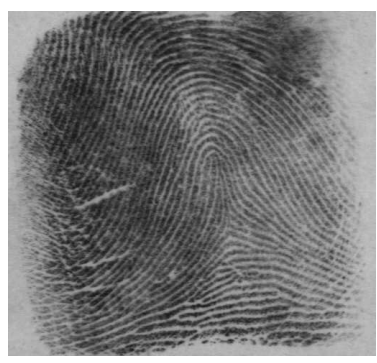

(a)

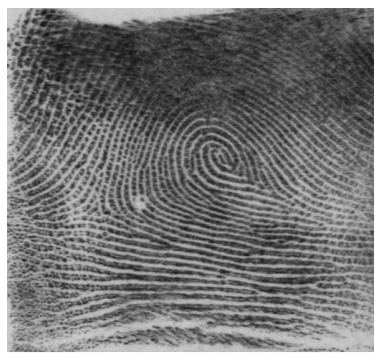

(b)

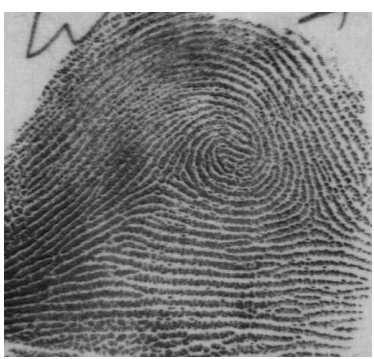

(c)

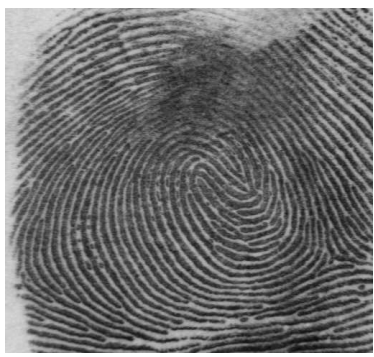

(d)

Figure 3.13: Small inter-class variability between classes $\mathbf{L}$ and $\mathbf{T}$ (a) $\mathbf{R}$ and $\mathbf{W}$, (b) and large intra-class variability between two prints of whorl class (c) and (d).

Unlike other classification schemes, our classifier does not require any other image processing techniques other than minutiae extraction.

\subsection{Analyzing classifier performance}

Above classification performance gives evidence for an indispensable relation between the class of the fingerprint and minutiae distribution. This adds an additional insight to individuality of fingerprints and may alleviate the public concern about the scientific basis for individuality of fingerprints. Use of minutiae for fingerprint classification can be considered as an example of super resolution where from bare minimum information about minutiae, we are interpolating global information of the fingerprint image. Though our classification result is inferior to the present state-of-the-art classification techniques [4], our intention is to show that it is feasible to assign a class to the fingerprint using its minutiae template alone. Mostly all the fingerprint databases are characterized by noisy fingerprints introduced at the time of acquisition. Some partial fingerprints may be lacking important global features. Fingerprints are non-uniformly distributed. The natural distribution of fingerprints into 5 classes is highly skewed - arch (3.7\%), tented arch (2.9\%), left loop (\%33.8), right loop (31.7\%), and whorl (27.9\%) [33]. Definition of each fingerprint class is both vague and complex. Even human experts having good experience cannot classify some ambiguous fingerprints. (About 17\% of 4000 images in NIST database 4 have 2 different ground truth tables.) The NIST 4 dataset has quite a few low quality and partial fingerprints (not capturing delta) Fingerprints have large intra-class variability i.e. prints of same class may have entirely different characteristics and small intra-class variability i.e. prints of different classes look similar as shown in Figure 3.13 . 
Most of the misclassifications represents the cases where ridges contributing to important pattern characteristics (e.g., recurving ridges) do not have minutiae. It can be seen visually from the minutiae plot in Figure 3.14 that it does not capture the curving back of ridges in the core region of a right loop thus resulting in a classification error. Some of the features like minutiae
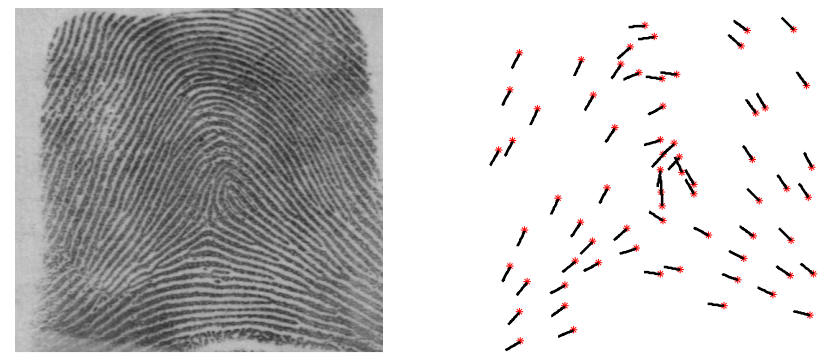

Figure 3.14: Atypical minutiae plot of a fingerprint of $\mathbf{R}$ class.

density, clusters of minutiae having homogeneous orientations are easily captured by human eye but it becomes a difficult problem when it comes to machine to do it. 


\section{Chapter 4}

\section{Reconstruction of fingerprints}

A minutiae template is a compact version of the original fingerprint image. Thus, traditionally, it has always been considered to be secure to store it in the database. In earlier chapters (Chapters 2, 3), we demonstrated that the minutiae template reveals significant amount of information such as ridge orientations and class of the parent fingerprint. In this chapter, we investigate the possibility of reconstructing fingerprints from minutiae. By the term 'reconstruction' of fingerprints, we mean, to regenerate fingerprint from its stored minutiae points. The reconstructed fingerprints can be used to spoof the authentication system and thus can be used for fraudulent purposes. This can be referred as masquerade attack on fingerprints. As described earlier (Chapter 1), Adler [14] has shown that, given access to the match score data, it is possible to reconstruct the enrolled face image using the face recognition template. Also, Hill [15] demonstrated that fingerprint like artefact can be generated from its stored minutiae points.

To the best of our knowledge, a technique for reconstructing fingerprint with minutiae points at desired locations has not been proposed in the literature. But, various approaches have been proposed for generating synthetic fingerprints. We first review the literature pertaining to synthetic fingerprint generation before discussing our scheme.

\subsection{Synthetic fingerprint generation}

Due to extensive use of fingerprint based authentication systems in commercial and civilian world, significant efforts are being made to design efficient fingerprint recognition algorithms. Usually these algorithms are evaluated on small proprietary databases. It is crucial to analyze and evaluate the matching performance of these algorithms on a larger database. Collecting large 
number of fingerprint images is expensive, tedious and time consuming. Also, it involves privacy issues as people may not be comfortable sharing their personal biometric data. These problems are alleviated by utilizing synthetic fingerprint generation software. A synthetic fingerprint is the ridge pattern that exhibits all the characteristics such as uniqueness and permanence as that of a real fingerprint. Two such commercially available synthetic fingerprint generation softwares are SFINGE [43] and Optel's Fingerprint Creator [44].

A detailed discussion of various approaches for synthetic fingerprint generation can be found in [4]. Three of them are discussed below.

1. Fourier Spectrum: Novikov and Glushchenko [45] proposed a ridge generation technique that operates in frequency domain. Firstly, an orientation image is artificially generated. The output image is initialized to a random image. For every pixel (x, y) in the random image, a 2D Fourier spectrum of local window centered around $(\mathrm{x}, \mathrm{y})$ is taken. Then, from the Fourier spectrum, the highest-energy along the normal to the local ridge direction at $(\mathrm{x}, \mathrm{y})$ (obtained from orientation image) is selected. The highest-energy harmonic in frequency domain corresponds to a 2D sinusoid in spatial domain. All such sinusoids are summed and the result is binarized. This procedure is repeated iteratively till a smooth image is obtained.

2. Fingerprint Creator : A Poland company named 'Optel' [44] has developed a software to generate synthetic fingerprints using a mathematical model based on minutiae and class information of the ridge pattern. Being a commercial software, no information about their fingerprint generation algorithm is currently available. The software takes various inputs such as class type, number of minutiae, density, rotation, translation in $\mathrm{x}$ and $\mathrm{y}$ direction, etc, but it does not take positions of minutiae points as input. Thus this method cannot be used to generate fingerprints having predefined minutiae locations.

3. SFINGE : This is a commercially available software developed by Cappelli et al. [43]. It consists of a model which characterizes the acquisition of fingerprint through an onlinesensor. Authors claim that, with few changes in the algorithm, fingerprint impressions using 'ink-technique' can also be generated. Their algorithm has 2 main steps. In the first step, a master fingerprint is generated and in the second step, multiple impressions of the same fingerprint are generated. Given certain input parameters such as fingerprint class, 
numbers and positions of singularities, 'seed points', etc., the algorithm can generate large fingerprint databases.

- Generation of Master Fingerprint : A master fingerprint is a ridge pattern that exhibits properties like uniqueness and permanence of a "synthetic finger" [4]. The generation of a master fingerprint is carried out in 4 steps:

(a) Fingerprint Shape Generation : Authors visually examined shapes of a large number of fingerprint images and prepared a simple model, based on four elliptical arcs and a rectangle. Depending upon the shape and size of the finger, the shape of a fingerprint is generally elliptical or circular. By changing a few parameters like the lengths of the minor and major axes of the ellipses, most of the shapes present in the real fingerprint images can be generated using this model.

(b) Orientation Image Generation : The orientation image generation is based on the technique proposed by Sherlock and Monro [20]. It requires the number and positions of core and delta points as input. In this model, the orientation image is represented in a complex plane, i.e., each location of the orientation image is considered as a complex number. Consider poles and zeros of the complex plane to be the coordinates where cores and deltas are located. Let $C_{i}, i=1,2, . . n_{c}$ and $D_{i}, i=1,2, . . n_{d}$ be the coordinates of cores and deltas respectively. Consider a point $\mathrm{z}=[\mathrm{x}, \mathrm{y}]$, then its orientation $\theta$ is obtained by,

$$
\theta=\frac{1}{2}\left[\sum_{i=1}^{n_{d}} \arg \left(z-C_{i}\right)-\sum_{i=1}^{n_{c}} \arg \left(z-D_{i}\right)\right],
$$

where, the function $\arg (x)$ yields the phase angle of the complex number $\mathrm{x}$. To generate an orientation image, first a fingerprint class is randomly chosen and accordingly, the singularity points are randomly selected. Due to absence of singularities in A class, this method cannot be used to generate arches. Instead, a simple sinusoidal function is used to generate an arch.

It was observed that this orientation model cannot determine the ridge flow accurately and, hence, SFINGE uses a variant of Sherlock and Monro's orientation model proposed by Vizcaya and Gerhardt [46] to provide more variations to the orientation image. This increases the number of degrees of freedom to cope with the variability in orientations. 
(c) Frequency image generation: This image decides the ridge frequency (density) in the generated fingerprint. Cappelli et al. observed a large number of real fingerprints to decide the overall ridge frequency. The overall frequency is chosen according to the distribution of ridge line frequency in real fingerprints. An average inter ridge distance of 9 pixels is selected (according to a 500 dpi sensor [4]). Authors observed that the regions above the northernmost loop and below southernmost delta have lower ridge line frequency than other regions in fingerprint. Thus the frequency is decreased in these regions. The frequency image is then randomly perturbed. Next, an averaging filter of a $3 \times 3$ size is used for smoothing the frequency image.

(d) Ridge Pattern Generation: SFINGE uses a very simple but powerful algorithm for generating ridge patterns. It uses space variant Gabor-like filters that are tuned to local ridge orientations and the frequency image. A white image is initialized with few random seeds. Then the Gabor filters are iteratively applied this white image, resulting in ridge-like patterns. Different types of fingerprint minutiae such as ridge endings, bifurcations, islands, etc. are generated automatically at random positions. The authors claim that the minutiae are generated from the ridge line disparity produced by local convergence/divergence of the orientation field and by frequency changes. Gabor filters have been used as an effective tool for fingerprint enhancement [47] and fingerprint matching [29]. But the authors successfully used these filters for fingerprint generation.

\section{- Generation of Synthetic Fingerprint Impressions from Master Fingerprint:}

Realistically, different impressions of the same finger captured by the same sensor, look significantly different. This is due to various factors like translation, rotation, non-linear distortion produced due to pressure, random noise, cuts, and bruises, etc. To synthetically generate various impressions of the same master fingerprint, following factors are varied:

(a) Variation in ridge thickness : The thickness of the ridge varies across various areas of the same fingerprint. It depends on the skin dampness and pressure applied when finger is put on a scanner. When the skin is wet or pressure is high, ridges appear thicker whereas when skin is dry and pressure is low, it appears 
thinner. The morphological operators such as dilation and erosion, are be applied for changing the thickness of the fingerprint ridges. The erosion operator aids in simulating low pressure or dry skin whereas the dilation operator in high pressure or wet skin. The size of the structuring element is varied to modulate the thickness of the ridge.

(b) Introducing non-linear distortion : The fingerprints are characterized by nonlinear distortions due to the pressure applied by the finger on the sensor surface during acquisition. Authors use a skin-distortion model to simulate this non-linear distortion in the generated fingerprint [48]. This model was originally proposed to re-map the minutiae points to cope with the non-linear distortion for improving the performance of fingerprint. In SFINGE, this model is applied to the whole image in order to simulate distorted impressions. Lagrangian interpolation is used to obtain smoothed gray-scale deformed images.

(c) Translation/Rotation : During fingerprint acquisition, the fingerprint image may not be centrally aligned. It might be translated and/or rotated. SFINGE generates various impressions by rotating and translating the master fingerprint.

(d) Addition of Noise : The non-uniform pressure of the finger against the sensor, the presence of small pores in the ridges, etc. make the fingerprint image noisy during acquisition. To introduce noise in the master fingerprint, small white blobs of various sizes and shapes are added.

(e) Background : SFINGE can generate backgrounds similar to that of the impressions captured by capacitive and optical sensors. A statistical model based on KL transform [49] is used for background generation. It requires a training set of certain background-only images. SFINGE uses Eigen value analysis for the background generation. Each background image is represented as a column vector. A mean vector of these images is computed. Then the mean vector is subtracted from each background image vector and a covariance matrix of all these vectors is computed. Using the significant eigen values, new background images are generated.

The process of fingerprint generation using SFINGE software is shown in Figure 4.1. 


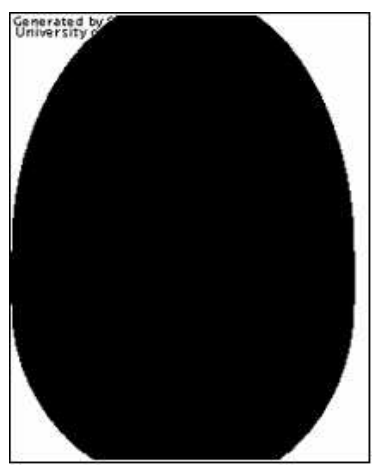

(a)

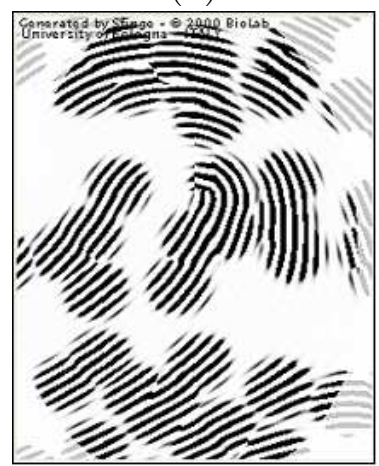

(d)

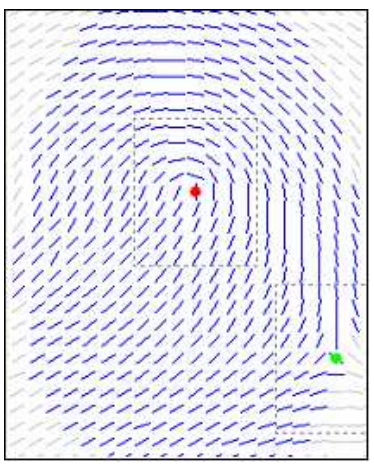

(b)

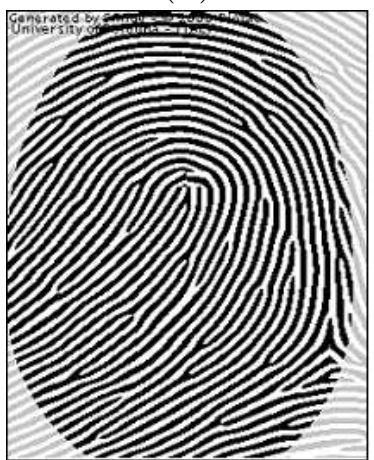

(e)

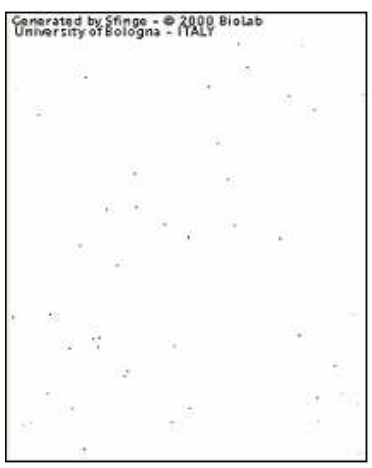

(c)

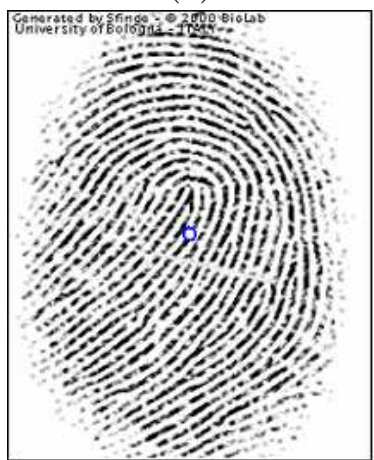

(f)

Figure 4.1: The process used by SFINGE to generate fingerprints: (a) Fingerprint shape generation, (b) Orientation image for a given set of core and delta points, (c) Output white image initialized with random seeds, (d) Generation of ridge pattern and minutiae initiated from seed points, (e) Synthetic L fingerprint, (f) Synthetic fingerprint with noise. (All figures are generated using SFINGE software). 
The fingerprint images generated by SFINGE appear very realistic. These fingerprint images were used to make a database namely DB4, for Fingerprint Verification Competitions (FVC2000 and FVC2002). In this competition, three other databases (DB1, DB2, and DB3) were obtained from real fingers. The performance of fingerprint matching algorithms on all four databases was observed and analyzed. The matching algorithms exhibit similar performance on DB4 as on the real fingerprint databases. This suggests that the fingerprints generated by SFINGE are realistic from the point of view of matching.

\subsection{Reconstructing fingerprints from minutiae points}

We assume that the stored template contains minimum information about the minutiae, i.e., their positions and orientations. The stored minutiae points give no direct information about the overall shape of the fingerprint, the width of the ridges, the inter-ridge distance, etc. Fingerprints are characterized by complex ridge patterns. Thus, given a minutiae template, a deterministic generation of fingerprint ridge pattern is certainly not an easy task. During the enrolment process, the information about the original fingerprint can be lost due to the following factors:

1. Generally to extract the minutiae points, various pre-processing techniques like image edge detection, binarization, thinning, etc. are employed. This may lead to significant loss of fingerprint information.

2. Also, most commercial softwares for minutiae extraction employ techniques to remove spurious minutiae. This may also result in the removal of some genuine minutiae. So the minutiae template might not contain all the significant minutiae

3. During acquisition of a fingerprint, due to improper finger placement, all the minutiae points may not be captured. Thus the stored minutiae template may not correspond to the entire fingerprint area.

Thus, it is is not feasible to recreate the original fingerprint image entirely using only the stored minutiae points. However, the template stores all the information required by the software for fingerprint matching. Therefore, if a digital artefact is recreated from the template, it can be used to spoof an authentication system. 
Given a minutiae template, we first estimate the orientation map from the minutiae distribution. We next proceed to generate the ridge structure of the parent fingerprint. We propose two schemes for fingerprint reconstruction. The first scheme uses space-variant Gabor-like filters based on SFINGE [43]. Although it generates ridge like patterns, it does not have control over the number and position of minutiae points in the reconstructed fingerprint. The second scheme uses streamlines and LIC (Line Integral Convolution). It enables us to generate minutiae at desired locations. The reconstructed fingerprint-like artefact has the appearance of the original fingerprint.

\subsubsection{Reconstructing fingerprints using Gabor-like filters}

We use the Gabor-like filter as used in [43] for reconstructing fingerprints. SFINGE uses class information and positions of cores and deltas to generate an orientation map using Sherlock and Monro's model [20]. Unlike SFINGE, we have information about minutiae positions and their orientations only. We use the estimated orientation map $\hat{\boldsymbol{\theta}}$ obtained using minutiae triplets. Each cell of this discrete map contains the average estimated orientation of a $13 \times 13$ local window of the parent fingerprint image. Note that, as this is a partial orientation map, some of its cells might not have any orientations. For each non-empty cell of $\hat{\boldsymbol{\theta}}$, ridges in the direction represented by the cell, are generated using a Gabor-like filter. The Gabor-like filter is tuned to the orientation contained by each cell. The filter is obtained by taking the product of a Gaussian by a cosine plane wave. A correction term is included to remove the $\mathrm{DC}$ component. The filter is given by,

$$
f(v)=\frac{1}{\sigma^{2}} e^{-\frac{\|v\|^{2}}{2 \sigma^{2}}}\left[\cos (\mathbf{K} \cdot v)-e^{-\frac{\sigma\|\mathbf{K}\|^{2}}{2}}\right]
$$

where, $\sigma^{2}$ is the variance of Gaussian which decides bandwidth of the filter, $\mathbf{K}=\left[k_{x}, k_{y}\right]$, is the wave factor of the plane wave. Here ' $\mathrm{v}$ ' is the location $(\mathrm{x}, \mathrm{y})$ in the output image where the filter is to be applied. The parameters $\sigma$ and $\mathbf{K}$ are adjusted using local ridge orientation and density. Let $\mathrm{O}(\mathrm{v})$ be the orientation of the pixel at location $(\mathrm{x}, \mathrm{y})$. The parameter ' $\mathrm{D}$ ' decides the ridge density in the generated fingerprint. The vector $\mathbf{K}$ is then found by the solution of following two equations.

$$
D=\sqrt{k_{x}^{2}+k_{y}^{2}}
$$




$$
\tan (O(v))=-\frac{k_{x}}{k_{y}}
$$

Our algorithm for fingerprint reconstruction uses a block by block approach. The block diagram of our reconstruction algorithm is shown in Figure 4.2. The main steps of our algorithm are as follows.

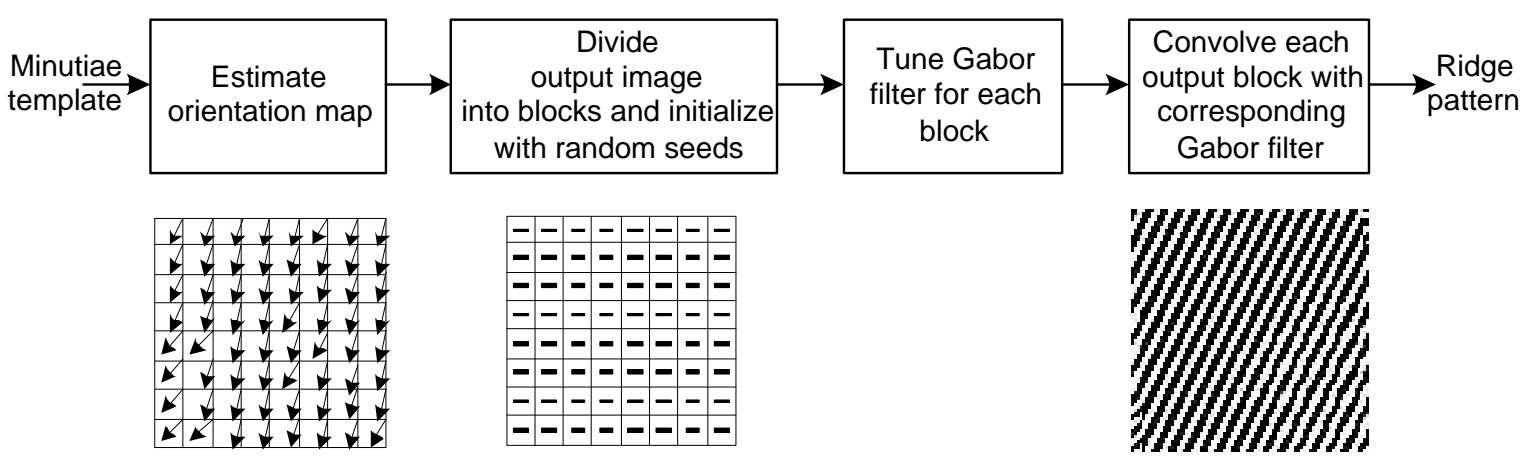

Figure 4.2: Block diagram showing the main stages of our reconstruction algorithm.

1. Compute orientation map : Given a minutiae template, we first estimate the orientation map $\hat{\boldsymbol{\theta}}$ using minutiae triplets as described in Chapter 2. Each non-empty cell of $\hat{\boldsymbol{\theta}}$ represents the average orientation for all the pixels of the corresponding block of the output image (Figure 4.2).

2. Initialize output image: Consider an initially white output image, divided into number of blocks, each of size $13 \times 13$ (same resolution as that of $\hat{\boldsymbol{\theta}}$ ) as shown in Figure 4.2. Each block of the output image is initialized with a noisy blob (seeds) at the center. The ridge pattern for each block of output image is generated using the estimated orientation of the corresponding cell of the orientation map.

3. Initialize filter parameters : For generating ridge patten for each corresponding block of the output image, the Gabor filter is tuned to the orientation of the corresponding cell of $\hat{\boldsymbol{\theta}}$. The parameter $\mathrm{O}(\mathrm{v})$ (Equation 4.4) is assigned the value of the orientation. The parameter $\mathbf{K}=\left[K_{x}, K_{y}\right]$ is computed by simultaneously solving Equations 4.3 and 4.4 . The bandwidth of filter $\sigma$ and the parameter $\mathbf{K}$ decide the ridge-width. For our experiments, we chose $\sigma=1.2$ and $D=1 / \sigma$. The ridge density $\mathrm{D}$ is adjusted according to $\sigma$ so that the filter does not contain more than 3 effective peaks [43]. It has been observed that the ridge 
width and ridge frequency are not uniform in a fingerprint. The minutiae template does not give any direct information about the ridge-widths and inter-ridge distances. Hence, we generate a fingerprint image with a constant ridge width and inter-ridge distance.

4. Ridge pattern generation : The ridge pattern is generated by iteratively convolving the image block with the Gabor filter. Figure 4.3 (b) and (e) show examples of two Gabor filters. The first Gabor filter is tuned to $45^{\circ}$ (Figure 4.3 (b)). Hence as shown in Figure 4.3 (c), the ridges for the output block are generated in $45^{\circ}$. Similarly, in the second block, ridges are generated in $191^{\circ}$. This procedure is repeated for all the blocks of the

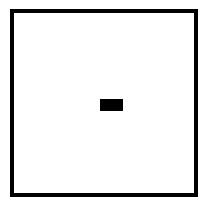

(a)

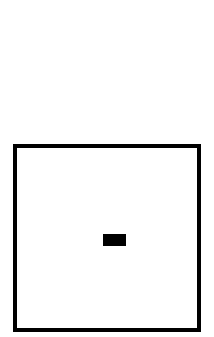

(d)

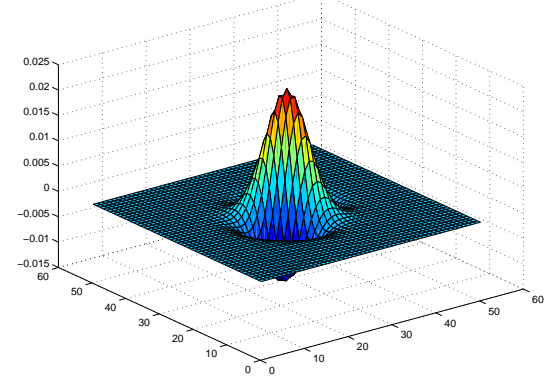

(b)

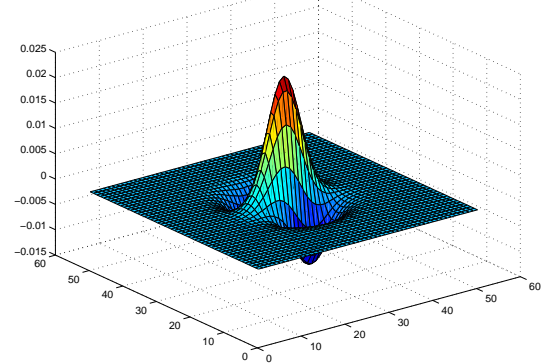

(e)

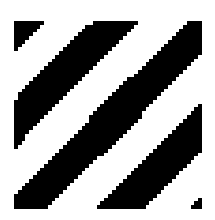

(c)

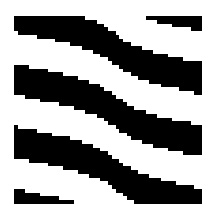

(f)

Figure 4.3: (a) Image block initialized with seeds, (b) A Gabor filter tuned to $45^{\circ}$, (c) Generated ridge pattern, (d) Image block initialized with seeds, (e) A Gabor filter tuned to 191º, (f) Generated ridge pattern.

output image. Figure 4.4 shows results of ridge generation after 1, 10 and 25 iterations. We observed that the output image does not change significantly after 25 iterations. We conducted experiments of ridge generation using various values of $\sigma$. The ridge pattern with lower value of $\sigma$ such as 0.2 , (Figure 4.5 (a)) cannot produce sufficient ridge width. On the other hand, if the value of $\sigma$ is too high, say 2 (Figure $4.5(\mathrm{c})$ ), the ridges become too thick. The optimal value we chose for our experiments was 1.2 (Figure 4.5 (b)). For all the three cases, the density $\mathrm{D}$ was set to $1 / \sigma$. 


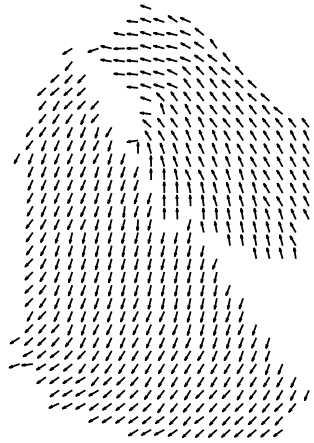

(a)

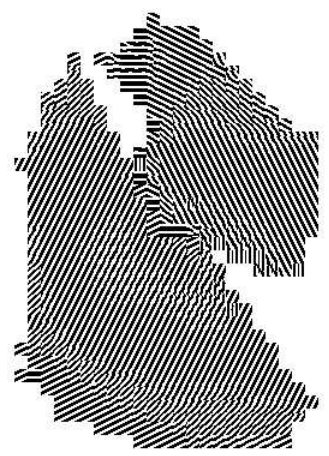

(d)

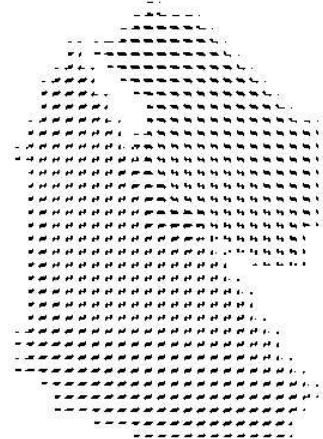

(b)

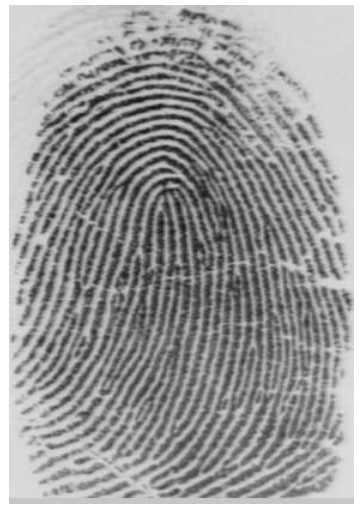

(e)

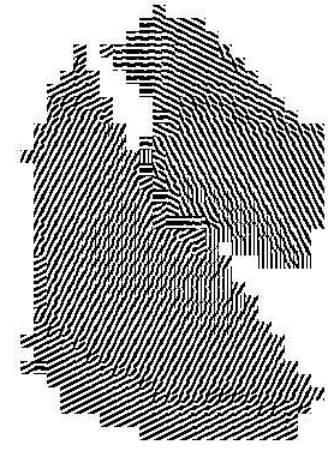

(c)

Figure 4.4: Fingerprint reconstruction: (a) Estimated orientation field for a $\mathbf{L}$ fingerprint, (b) Ridge generation after 1 iteration, (c) Ridge generation after 10 iterations, (d) Reconstructed ridge pattern after 25 iterations, (e) Original $\mathbf{L}$ fingerprint. 


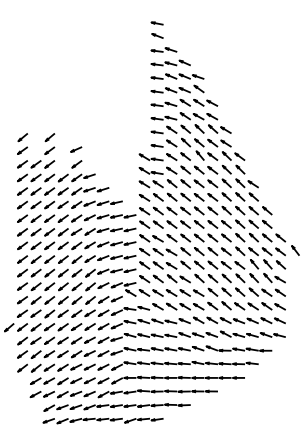

(a)

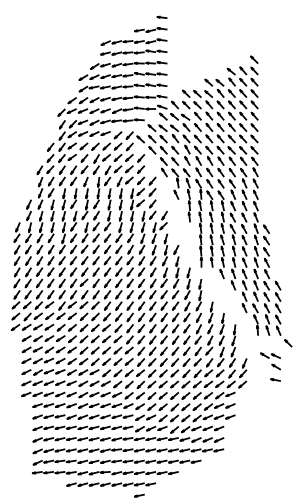

(a)

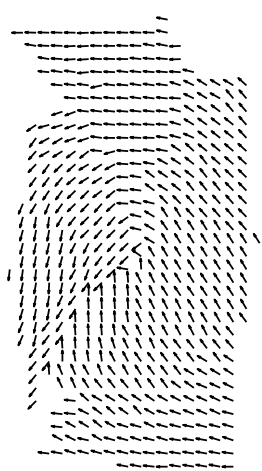

(a)

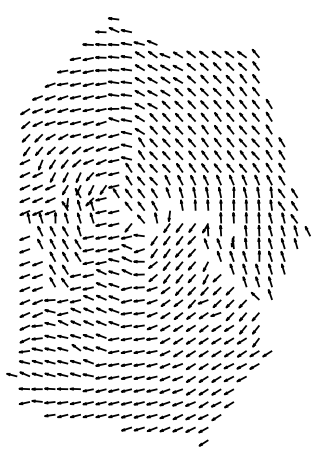

(a)

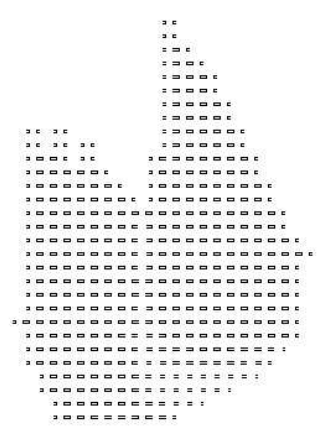

(b)

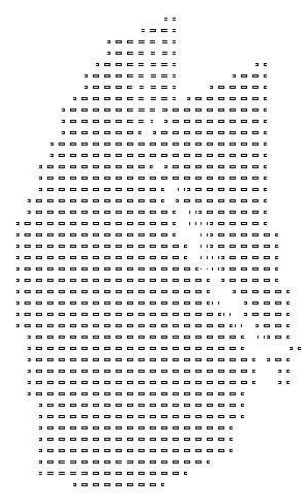

(b)

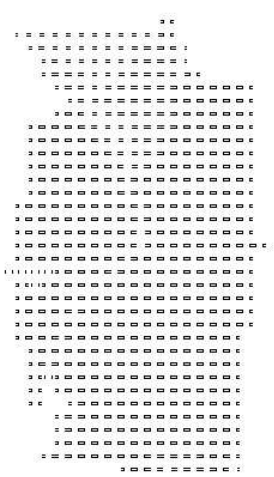

(b)

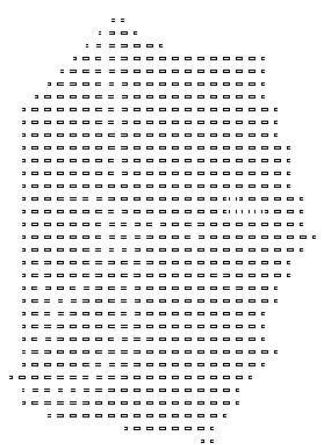

(b)

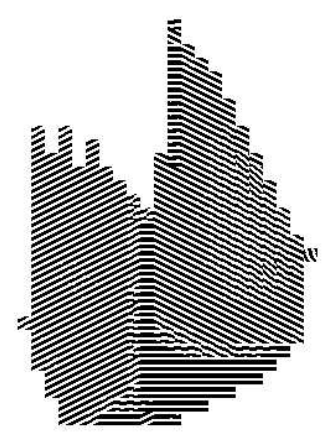

(c)

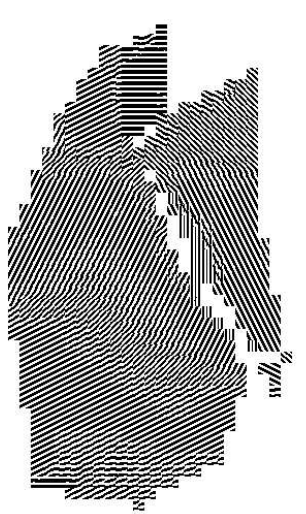

(c)

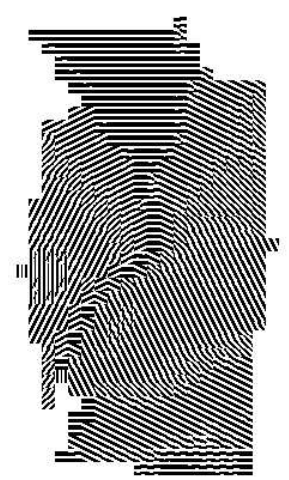

(c)

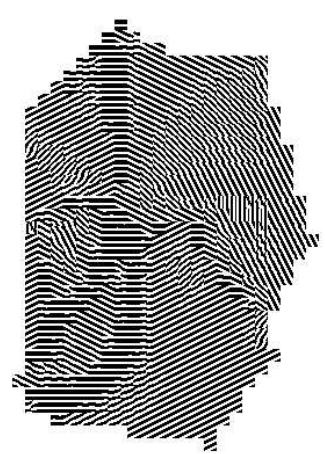

(c)

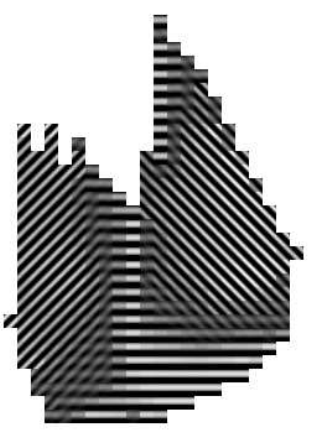

(d)

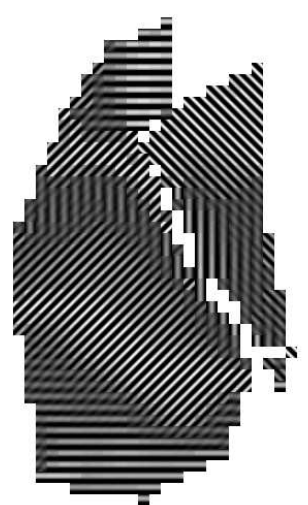

(d)

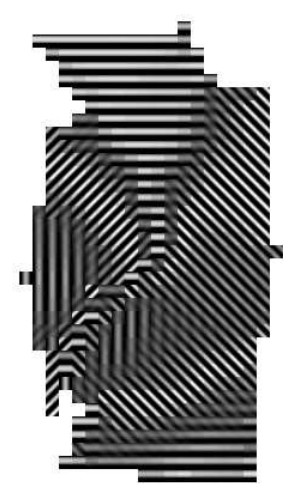

(d)

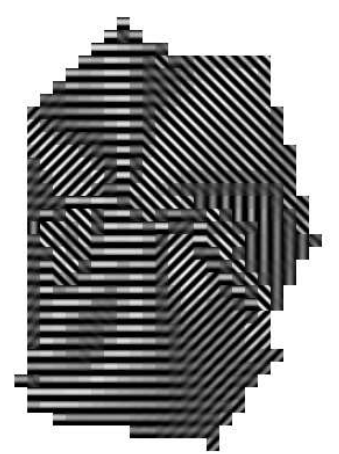

(d)

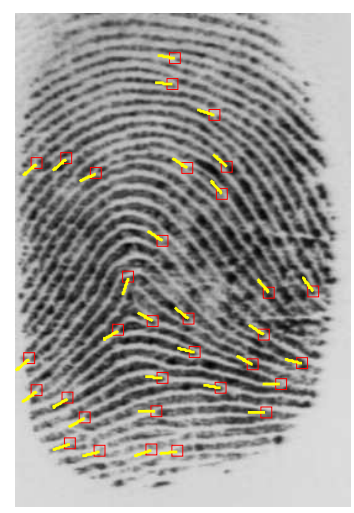

(e)

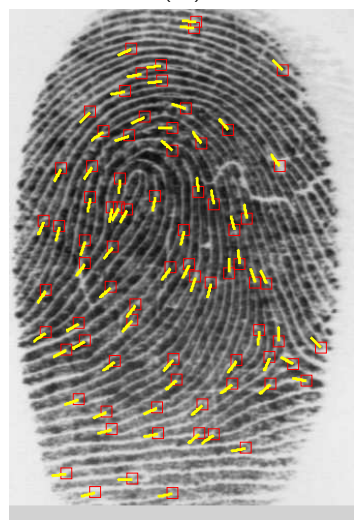

(e)

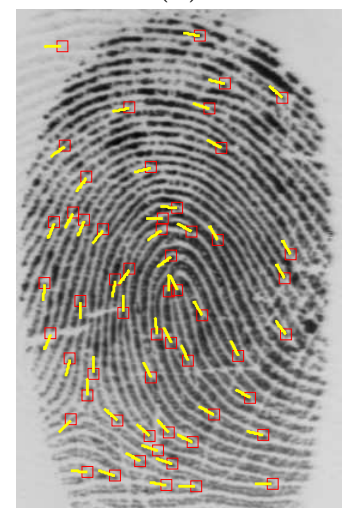

(e)

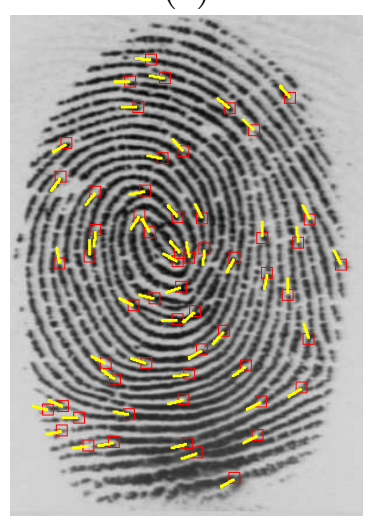

(e)

Figure 4.5: (a) Estimated Orientation Map $\hat{\boldsymbol{\theta}}$, Output of ridge generation with (b) $\sigma=0.2$, (c) $\sigma=1.2$, (d) $\sigma=2$, (e) Original fingerprint for classes $\mathbf{A}, \mathbf{L}, \mathbf{R}$ and $\mathbf{W}$. 
We observed that for $\sigma=1.2$, if we set $\mathrm{D}$ value to be very small such as 0.1 then the convolution resulted in a black image (Figure 4.6 (a)). If this value is larger say 5, 10 or 15 then the ridge are generated with larger density (Figure $4.6(\mathrm{c}),(\mathrm{d}),(\mathrm{e})$ ). We observed the value $\mathrm{D}=1 / \sigma$ resulted in the acceptable ridge density. More results of reconstruction for other fingerprint classes are

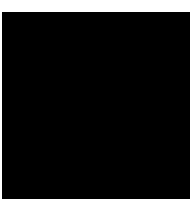

(a)

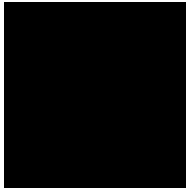

(b)

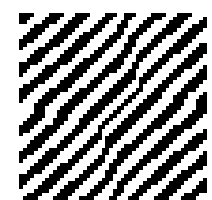

(c)

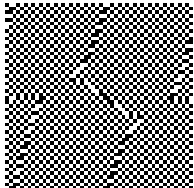

(d)

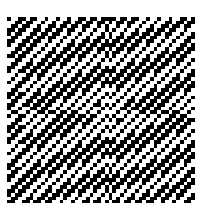

(e)

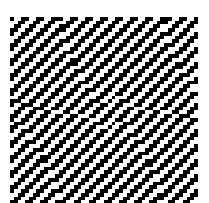

(f)

Figure 4.6: Output of ridge generation with (a) $D=0.1$, (b) $D=0.2$, (c) $D=1 / \sigma$, (d) $D=5$, (e) $D=10$, (f) $D=20$.

shown in Figure 4.7. In this method, we do not have control over the minutiae generation.

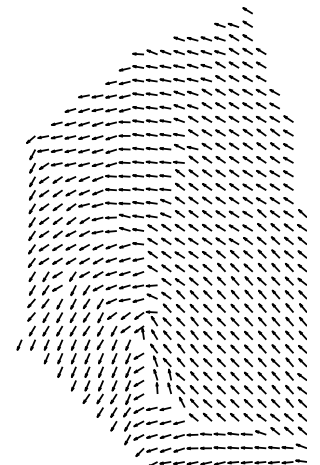

(a)

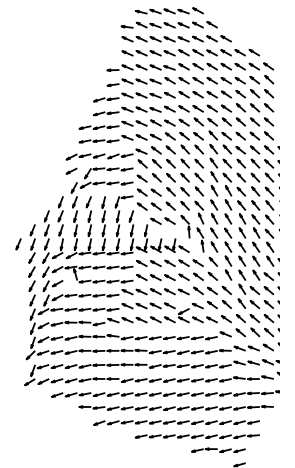

(a)

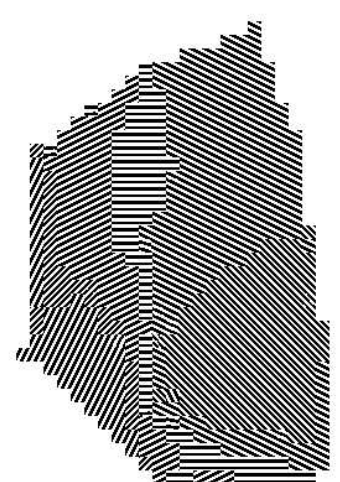

(b)

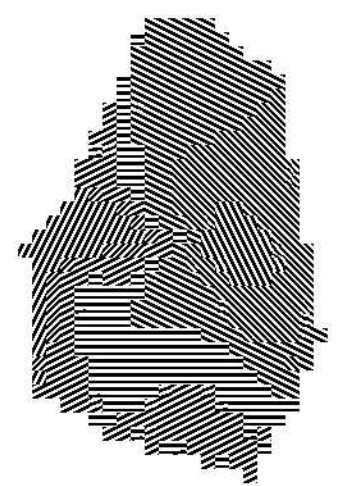

(b)

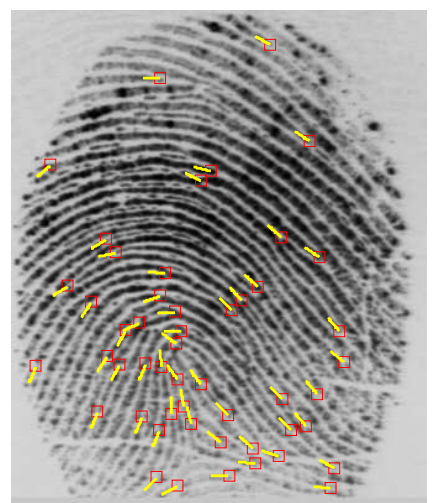

(c)

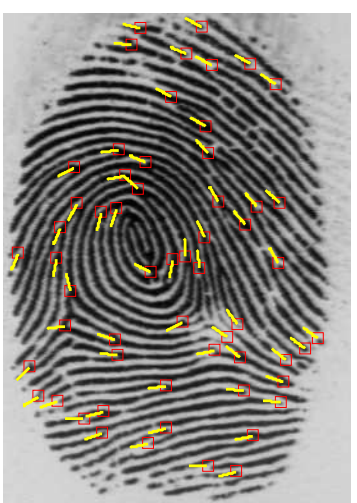

(c)

Figure 4.7: (a) Estimated orientation map $\hat{\boldsymbol{\theta}}$, (b) Reconstructed fingerprint, (c) Original fingerprint of $\mathbf{T}, \mathbf{R}$, and $\mathbf{W}$ respectively. 


\subsubsection{Reconstructing fingerprints using Streamlines and LIC}

Streamlines and LIC (Line Integral Convolution) have been widely used for imaging arbitrary two- and three-dimensional vector fields [50, 51]. Imaging vector fields has applications in science, engineering, art, image processing, image rendering, animation and special effects. Examples of vector fields include velocities of wind currents (e.g., for weather forecasting), results of fluid dynamics simulation, blood flow, components of stress and strain in materials, etc. Hence, algorithms that can image the directional information have wide application across both scientific and artistic domains.

Here, we propose a novel application of streamlines and LIC for reconstructing fingerprints. Given a vector field, streamlines are the curves that are tangential to the vector field at every point. These are also called as integral curves as they are generated by integrating the vector field. LIC is basically a texture synthesis technique that is used to visualize 2D data. Cabral and Leedom [50] proposed LIC for imaging vector fields and to produce novel special effects such as motion blurring in images. Figure 4.8 shows main steps for visualizing vector filed using streamlines and LIC. For a given vector field, first the streamlines are generated. Using streamlines and a spatially uncorrelated white noise image, LIC computes intensity values for the coordinates of each streamline. It applies a one-dimensional filter to blur the noise image along the streamlines resulting in a texture image which aids in the clear visualization the vector field.

A fingerprint is an oriented texture pattern and, therefore, the use of streamlines and LIC for generating the ridge structure is quiet appropriate; streamlines are used to generate the thinned ridges whereas LIC is used to impart texture-like appearance to the ensuing ridges. The main stages regenerating fingerprints are shown in Figure 4.9 .

1. Computing orientation map $\hat{\boldsymbol{\theta}}$ : Given a minutiae template, we first obtain the estimated orientation map $\hat{\boldsymbol{\theta}}$ using minutiae triplets described in Chapter 2 .

2. Constructing Streamlines Using $\hat{\boldsymbol{\theta}}$ : Consider an estimated orientation map $\hat{\boldsymbol{\theta}}: R^{2}=>$ $R,(x, y) \rightarrow \hat{\theta}(x, y)$, where $(x, y)$ is an arbitrary location in $\hat{\boldsymbol{\theta}}$. A streamline is a path in the orientation map $\hat{\boldsymbol{\theta}}$, whose tangent vectors coincide with $\hat{\boldsymbol{\theta}}$ (Figure 4.10 (a)). Let $P$ be a point in the orientation map $\hat{\boldsymbol{\theta}}$ and $S$ be a streamline passing through $P$. Let the curvature 


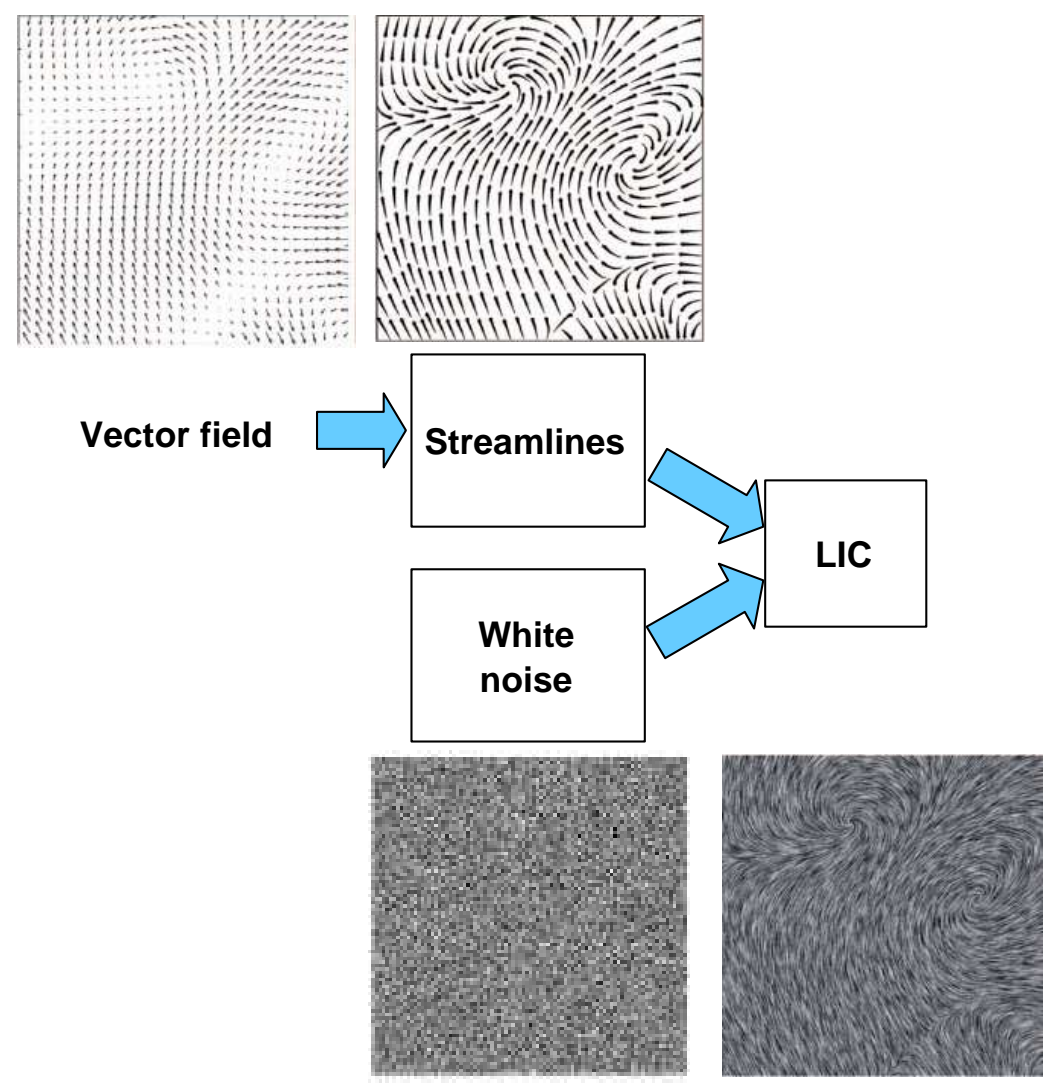

Figure 4.8: Block diagram showing visualizing vector field using LIC.

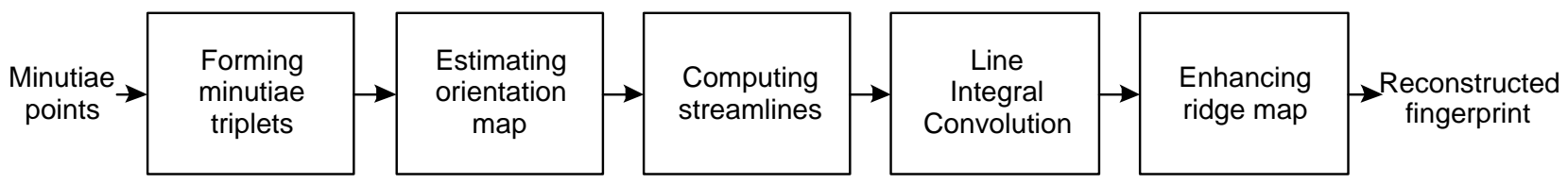

Figure 4.9: Block diagram showing the main stages of the proposed fingerprint reconstruction algorithm. 


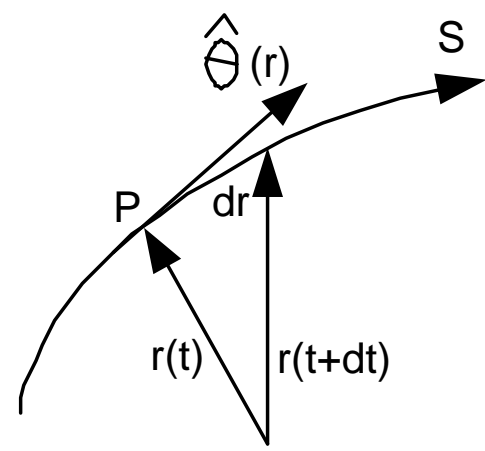

(a)

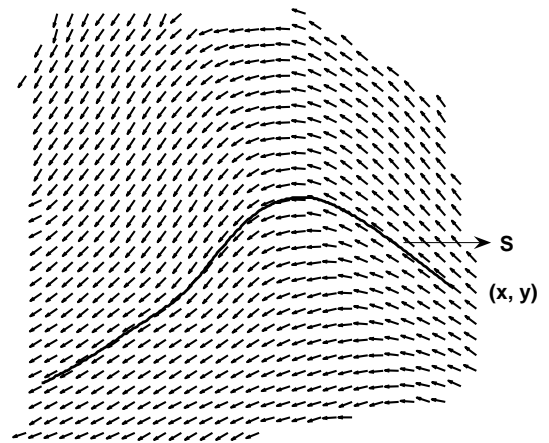

(b)

Figure 4.10: (a) A streamline $S$ tangent to orientation field $\hat{\boldsymbol{\theta}}$, (b) Streamline originating from a seed point $(\mathrm{x}, \mathrm{y})$ in the vector field $\hat{\boldsymbol{\theta}}$

at point $P$ be due to a circle of radius $r(t)$. Then the tangent $d r$ at point $P$ is given by,

$$
\frac{d r}{d t}=\hat{\theta}[r(t)]
$$

where $d t$ represents the next position. A numerical integration technique or interpolation techniques can be used to solve this ordinary differential equation (ODE). Integrating equation 4.5 yields,

$$
r(t)=r(0)+\int_{0}^{t} \hat{\theta}(r(t)) d t
$$

With the help of equation 4.6, a streamline can be initiated from an initial seed point and grown using small steps of $d t$. There are various numerical integration techniques that can be used to solve 4.6: (a) fixed step size integrators such as Euler; (b) non constant or adaptive step size integrators such as cubic Hermite-interpolation; and (c) continuous integration methods such as the fifth order Runge-Kutta integrator with adaptive step size. In adaptive step size schemes, the value of $d t$ is determined at each step of the integration in order to keep the relative error within a specified tolerance. The accuracy of the ODE solver determines the exactness of the resulting streamline. Consider $d t=t_{i}-t_{i-1}$, then the error in streamline construction is $O(d t)^{n}$, where $\mathrm{n}$ is the order of the ODE solver.

If we solve the above ODE for $\hat{\boldsymbol{\theta}}(x, y)$ with a seed point as $(x, y)$, we get a streamline as shown in Figure 4.10 (b). In order to generate streamlines for constructing ridge lines, the 
following issues have to be addressed: (a) selection of seed points, (b) criteria for streamline termination, (c) distance between adjacent streamlines, and (d) generation of minutiae in pre-determined positions.

(a) Seed point selection : The seed points are the pre-specified points in an orientation map $\hat{\boldsymbol{\theta}}$ from which the streamlines are originated. Figure 4.10 (b) shows an example of seed point $(\mathrm{x}, \mathrm{y})$ from which a streamline is originated. Here, the seed point can be considered to be a minutia (ridge ending). Thus, in order to generate minutiae at desired locations, we use the minutiae locations as seed points. However, using only minutiae as seed points results in the sparse distribution of streamlines over the image plane (Figure $4.11(\mathrm{~b})$ ). Thus, we also use the boundary points of $\hat{\boldsymbol{\theta}}$ as seed points. We observed that streamlines generated using only the border points as seed points capture the global shape of the parent fingerprint as shown in Figure 4.11 (c). Thus for fingerprint reconstruction, we use both, minutiae points and border points of $\hat{\boldsymbol{\theta}}$ as seed points.

The minutiae points in fingerprints tend to occur in clusters [26]. In such high minutiae-density areas, seed points will occur in close proximity resulting in a clutter of streamlines in the local region. To solve this problem, we place all the seed points $S_{i}, \mathrm{i}=1 \ldots \mathrm{k}+\mathrm{n}$ (where $\mathrm{k}$ and $\mathrm{n}$ are the number of border points and minutiae respectively) on a lattice of cell size $D_{s} \times D_{s}$ such that two seed points are at least $D_{s}=10$ distance apart from each other as shown in Figure 4.12 (a). Due to this mapping, a single grid point may correspond to multiple minutiae points. This prevents the excessive proliferation of streamlines in a local region.

(b) Streamline construction : While most particle tracking algorithms (e.g., [52]) use various numerical integration techniques to solve equation 4.6, we used the 'stream3c' function available in the Volume Visualization Toolbox of Matlab(7.0). It uses a linear interpolation scheme for constructing streamlines. Once a streamline is initiated from a seed point, the next position is obtained by updating its current position based on the orientations of the immediate neighbors of the seed point. The streamline is terminated if (a) it encounters a boundary point in the grid, or (b) if it arrives in the vicinity of a minutia point. When a streamline is within a predetermined distance from 


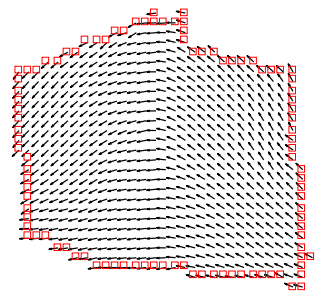

(a)

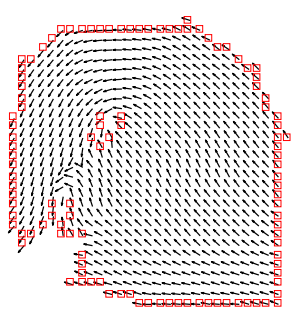

(a)

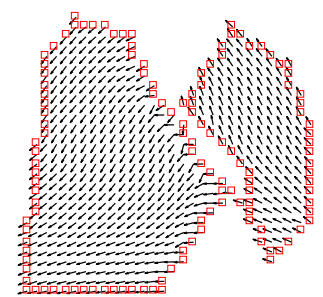

(a)

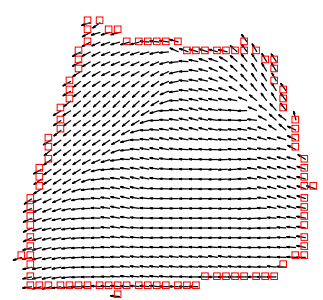

(a)

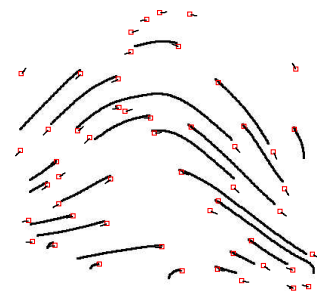

(b)

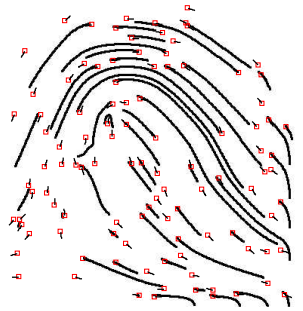

(b)

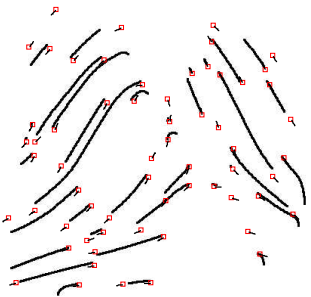

(b)

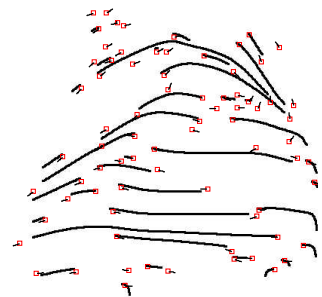

(b)

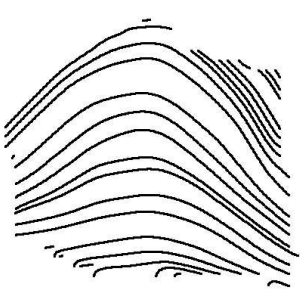

(c)

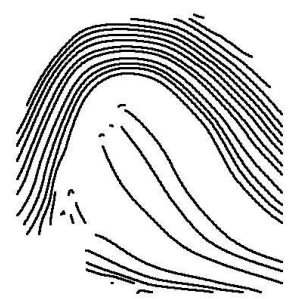

(c)

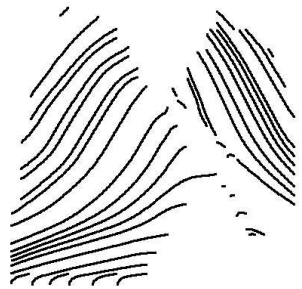

(c)

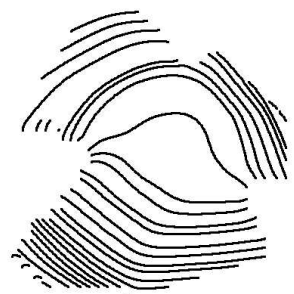

(c)

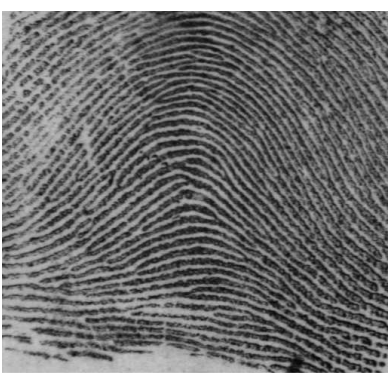

(d)

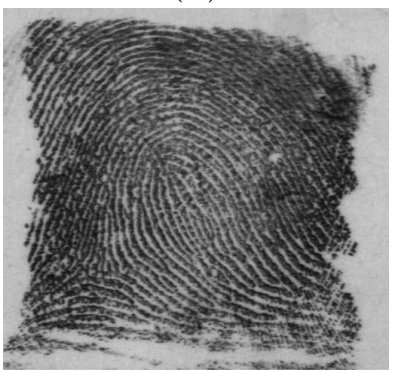

(d)

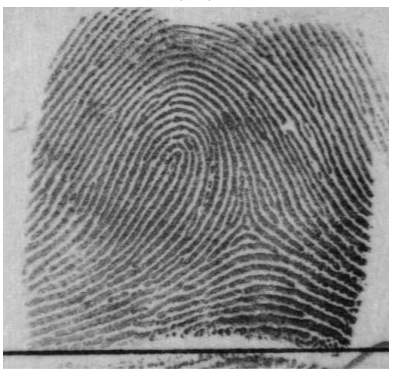

(d)

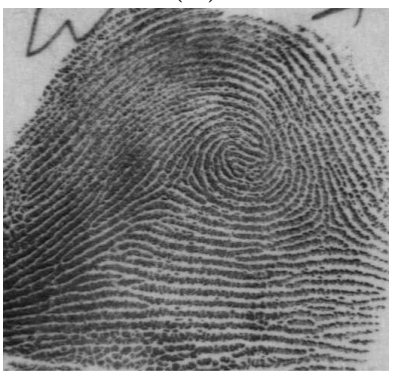

(d)

Figure 4.11: (a) Estimated orientation map $\hat{\boldsymbol{\theta}}$ with border points marked in 'red' color, (b) Streamlines generated from minutiae points as seed points, (c) Streamlines generated using border points as seed points for classes, (d) Original fingerprint for $\mathbf{A}, \mathbf{R}, \mathbf{L}, \mathbf{W}$ respectively. 


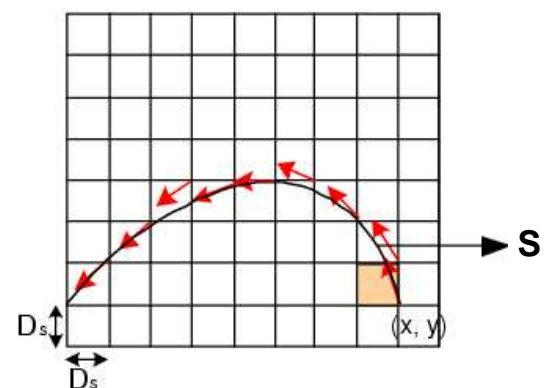

(a)

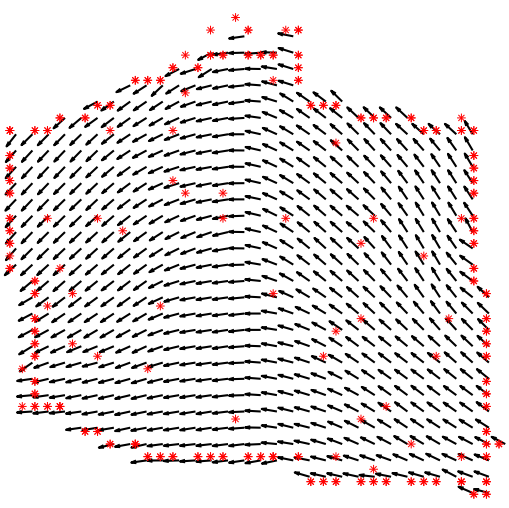

(b)

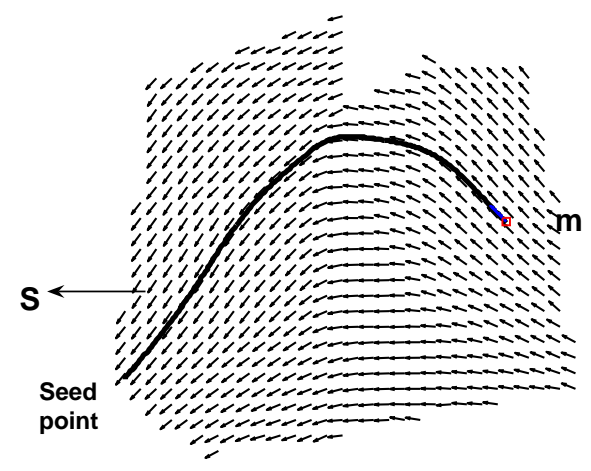

(c)

Figure 4.12: (a) Lattice of seed points $\left(D_{\text {seed }}=10\right)$, (b) Seed points 'S' overlaid on the orientation field, $\hat{\boldsymbol{\theta}}$, of an arch, (c) Streamline terminated near a minutia ' $\mathrm{m}$ '.

a minutia point (in our case, 5 pixels apart), it is terminated as shown in Figure 4.12 (c). So a minutia (ridge ending) is generated if either a streamline initiates from that point or another streamline appears in its proximity. In the current implementation, we generate only ridge endings at the desired minutiae locations since it is assumed that the 'type' of the minutia is not stored in the template.

It has been observed in the literature that a constant density of seed points do not necessarily ensure an even distribution of the streamlines [50]. The generated streamlines may not be evenly distributed, resulting in a frenzy of streamline activity in certain local regions. While several elegant techniques exist for creating evenly-spaced streamlines (see [53], for example), we employ a simple technique to control inter-ridge distances $\left(D_{r}\right)$. We generate ridges with uniform inter-ridge distances over the entire fingerprint image as we assume that the minutiae template does not give any information about the inter-ridge distance of the parent fingerprint. During streamline construction, if a streamline is in the proximity of another streamline $\left(D_{r}=5\right)$, then it is discarded. This avoids the cluttering of streamlines in local regions (Figure 4.13).

Figure 4.14 shows the results of streamline generation for different fingerprint classes.

3. Generating ridge structure using LIC: As described above, using streamlines, thin ridge lines for the estimated orientation map $\hat{\boldsymbol{\theta}}$ can be generated. The intensity values of ridge pixels shown in Figure 4.14 (c) are single valued i.e. ' 0 '. In order to impart 


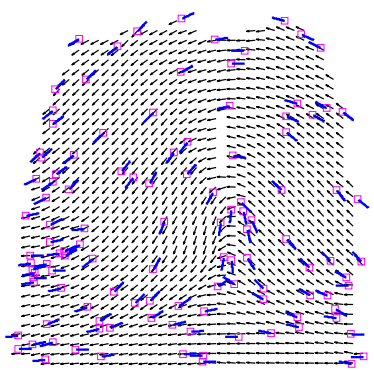

(a)

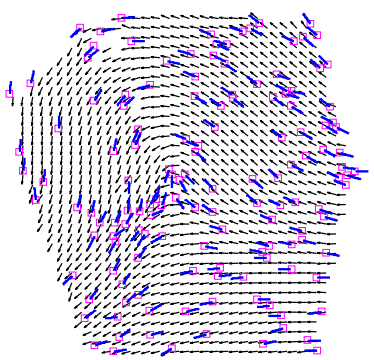

(a)

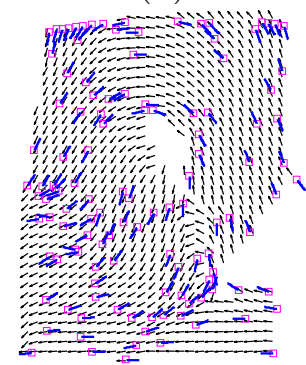

(a)

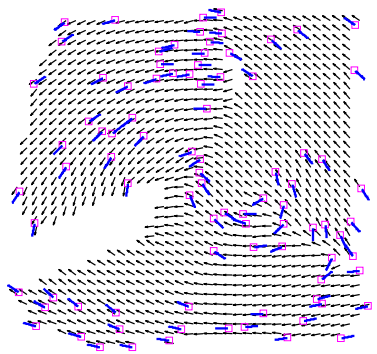

(a)

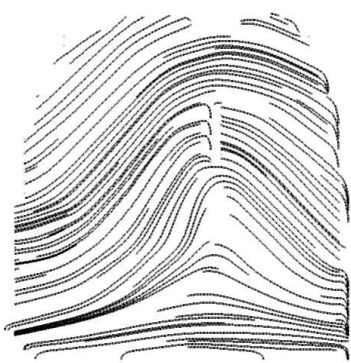

(h)

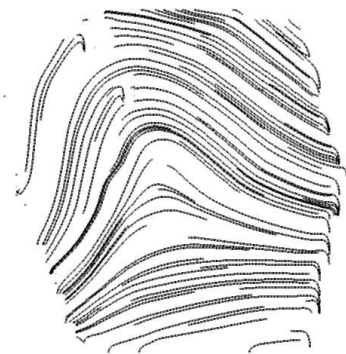

(b)

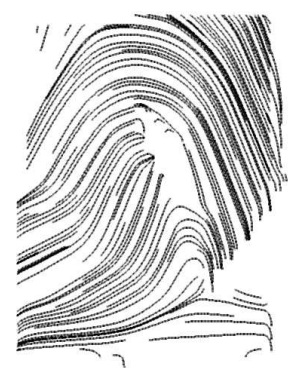

(b)

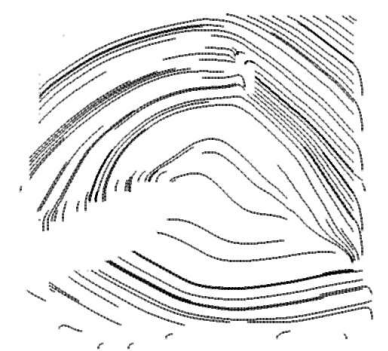

(b)

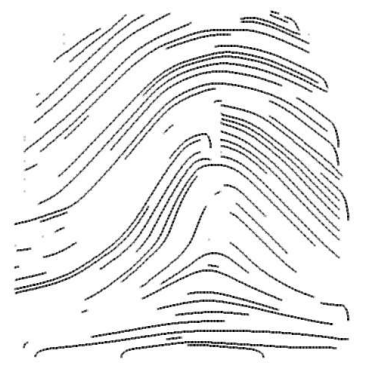

$(r)$

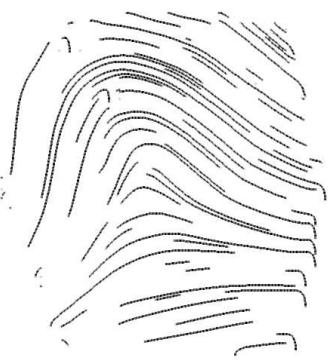

(c)

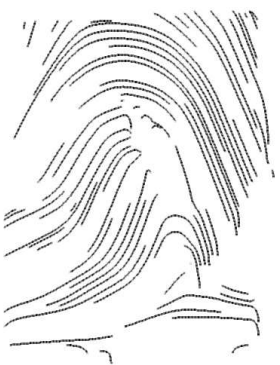

$($ )

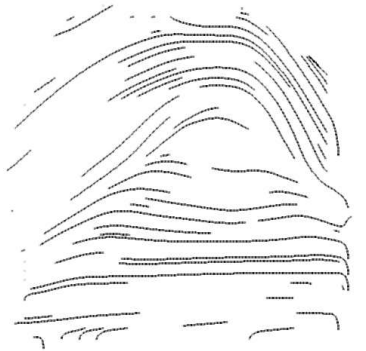

(c)

Figure 4.13: (a) Estimated orientation map $\hat{\boldsymbol{\theta}}$, (b) Streamlines generated without controlling $D_{r}$, (c) Streamlines generated by controlling $D_{r}=5$, for $\mathbf{A}, \mathbf{R}, \mathbf{L}, \mathbf{W}$. 


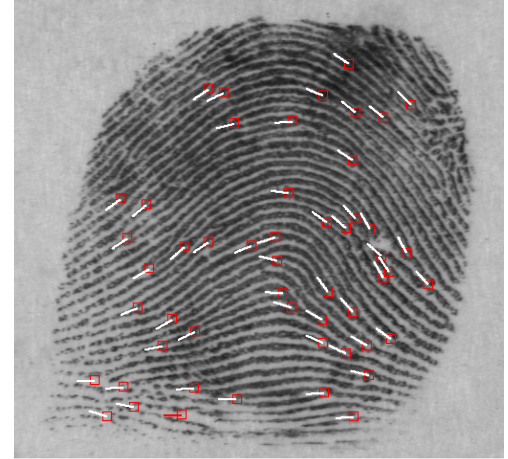

(a)

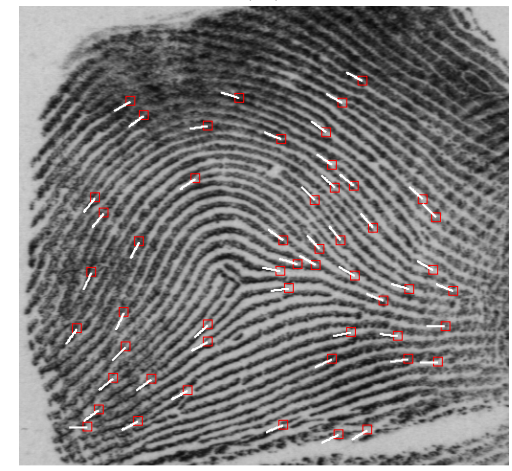

(a)

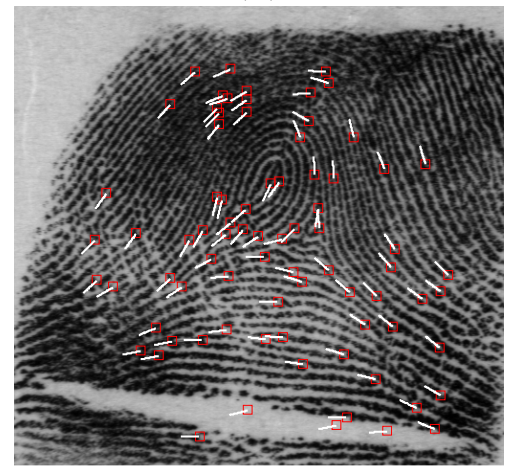

(a)

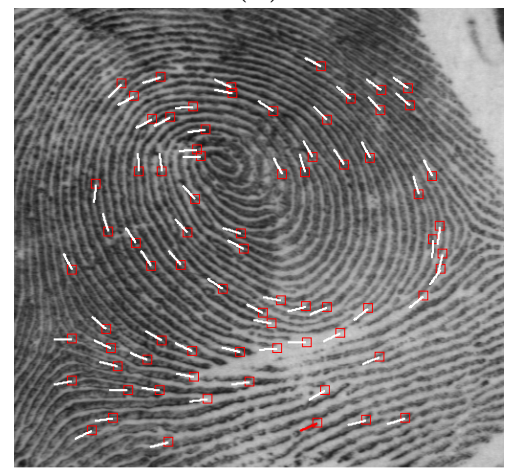

(a)

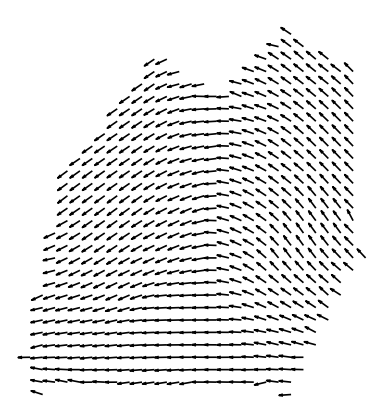

(b)

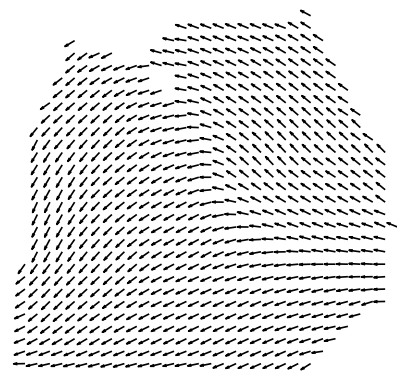

(b)

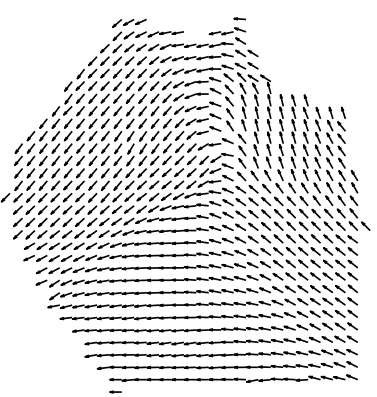

(b)

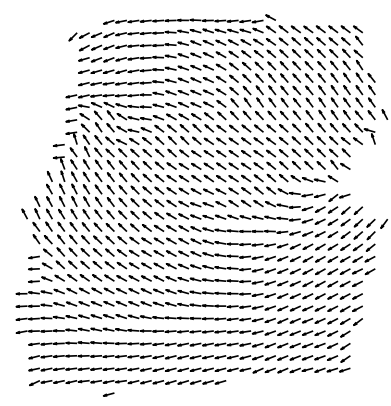

(b)

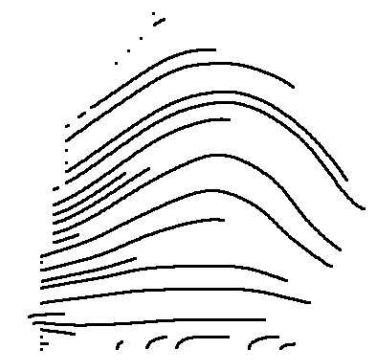

(c)

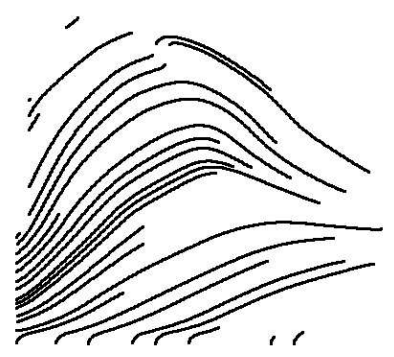

(c)

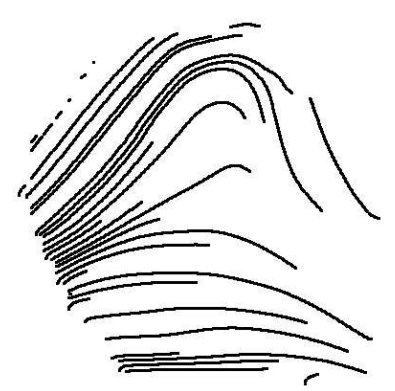

(c)

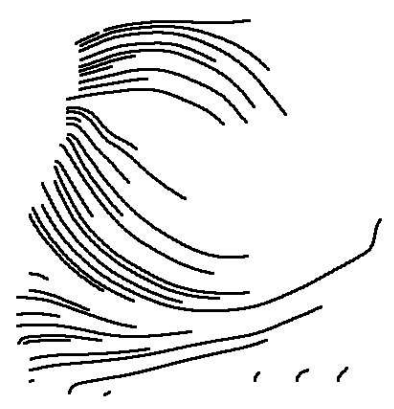

(c)

Figure 4.14: (a) Minutiae plot, (b) Estimated orientation map $\hat{\boldsymbol{\theta}}$, (c) Streamlines 
texture-like appearance to the ridges, we use Linear Integral Convolution (LIC). Given a streamline $S$, the LIC technique involves calculating the intensity of all pixels constituting the streamline. It locally blurs an uncorrelated input texture image such as white noise, along the path of the streamlines to impart a dense visualization of the flow field. Consider a pixel at location $x_{0}=S\left(p_{0}\right)$ on the streamline. Then its intensity is computed using one-dimensional filtering as,

$$
I\left(x_{0}\right)=\int_{p_{0}-L}^{p_{0}+L} k\left(p-p_{0}\right) T S(p) d p
$$

where, $T$ is a texture (white noise image). The kernel, $k$, is a one-dimensional low-pass filter with $L=25$ pixels. The convolution results in the generation of ridge-like patterns whose orientations correspond to the predicted vector field (Figure 4.15). The filter length ' $L$ ' determines how much the texture is smeared in the direction of the vector field. Due

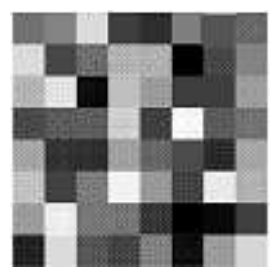

(a)

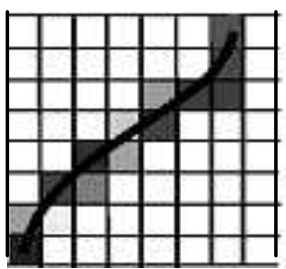

(b)

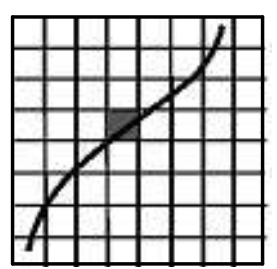

(c)

Figure 4.15: Lending texture to a streamline using LIC: (a) White noise image, T, (b) Convolving $\mathrm{T}$ with a streamline, (c) Result of LIC.

to LIC, the binary image generated using streamlines is converted to a gray scale image (Figure $4.16(\mathrm{~b})$ ).

4. Enhancing the ridge map: Although LIC provides texture-like appearance to the thin ridges (streamlines), the ridges are still one-pixel thick. In order to increase the ridge width, we first use a lowpass filter to smooth the texture image generated using LIC and then perform histogram equalization of the ridge structure for contrast enhancement as shown in Figure $4.16(\mathrm{c})$.

\subsubsection{Comparing two reconstruction schemes}

Figure ?? compares the two techniques for fingerprint regeneration using: (a) Gabor-filters, and (b) Streamlines and LIC. Unlike Gabor filters, with streamlines and LIC, the reconstructed 


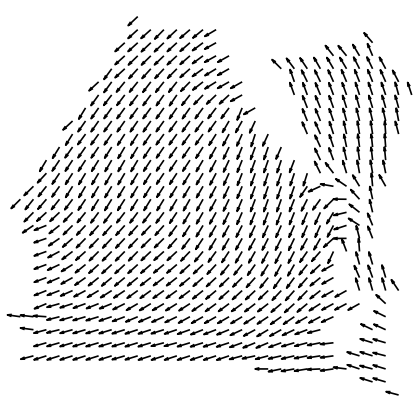

(a)

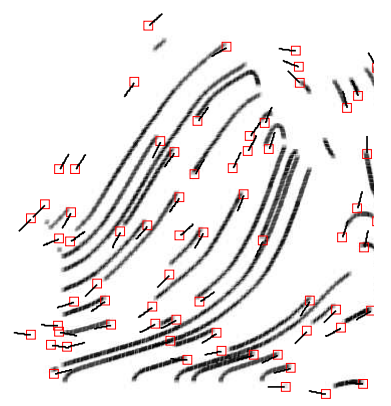

(b)

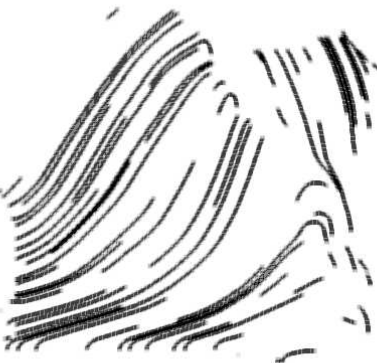

(c)

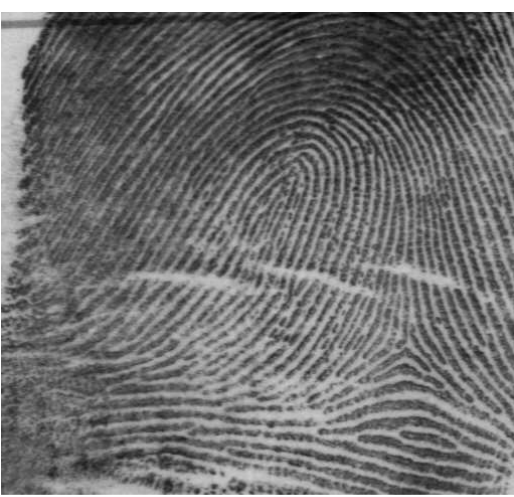

(d)

Figure 4.16: (a) Estimated orientation map $\hat{\boldsymbol{\theta}}$, (b) Result of applying LIC, (c) Enhanced fingerprint using a $5 \times 5$ lowpass filter, (d) The original fingerprint.

ridge pattern exhibits shape characteristics of the parent fingerprint significantly. It also enables to place the minutiae at desired locations and with desired orientations in the reconstructed fingerprint.

The reconstructed fingerprints using minutiae are partial. This is because a minutiae template may not capture all the global properties of the parent fingerprint. We demonstrated that it is indeed possible to reconstruct the at least those parts of the fingerprint which are used for authentication.

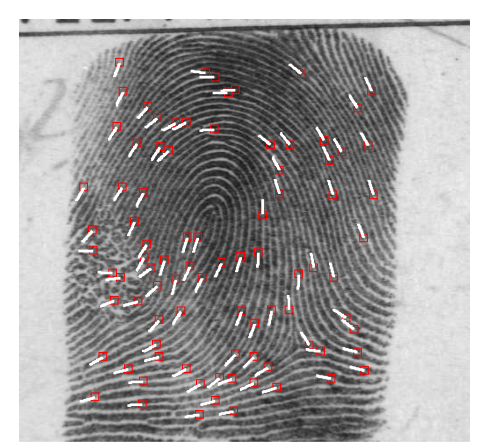

(a)

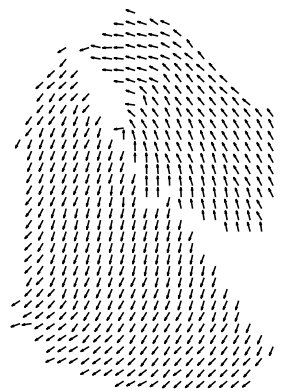

(b)

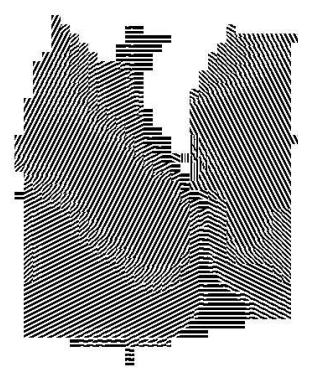

(c)

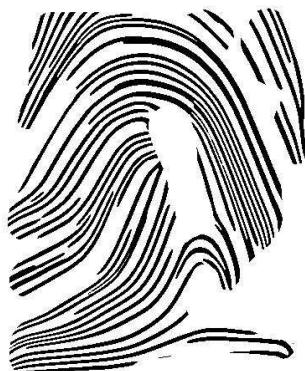

(d)

Figure 4.17: (a) Minutiae plot, (b) Estimated orientation map $\hat{\boldsymbol{\theta}}$, (c) Regenerated fingerprint using Gabor filters, (d) Regenerated fingerprint using LIC 


\subsubsection{Spoofing of fingerprint authentication system}

Figure 4.18 shows an overlay of original and reconstructed fingerprints of $\mathbf{A}$ and $\mathbf{L}$. Visually, it is evident that the reconstructed fingerprints are consistent with the underlying ridge structures. We conducted few experiments to verify if the reconstructed ridge structure may be used to

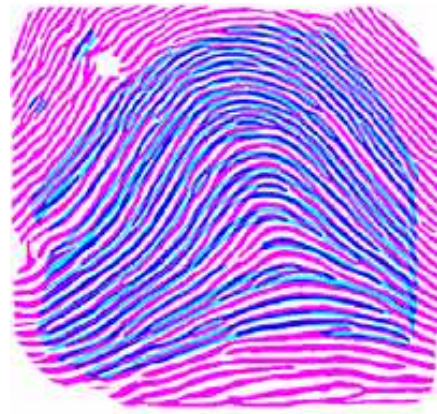

(a)

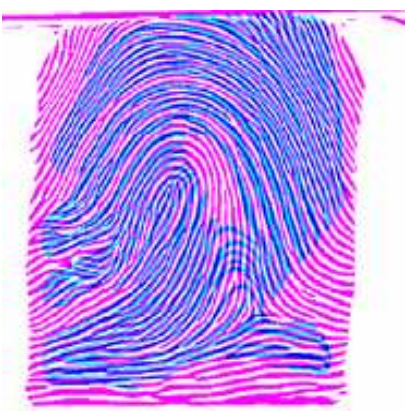

(b)

Figure 4.18: Overlay of original (magenta) and reconstructed (blue) fingerprints for two minutiae templates.

spoof an authentication system. The authentication system used for fingerprint matching was VeriFinger 4.1 SDK, developed by Neurotechnologija ${ }^{1}$. The software has the ability to process fingerprint images (apart from the live samples captured by sensor system) supported by the SDK. Given an input fingerprint image, the software first enhances the image and converts it into a binary image using certain image processing routines (Figure 4.19), extracts minutiae from the enhanced images and stores minutiae positions $(\mathrm{x}, \mathrm{y})$ and the orientations $(\theta)$ in the template. It is evident from Figure 4.19 (d) that the enhancement algorithm employed by the software also removes certain parts of the ridges of the reconstructed fingerprint in the regions with low ridge density. On matching, it releases the match (similarity) score. Being a commercial software much information about its minutiae extraction and matching algorithm is not available. To spoof the authentication system, we used NIST-4f and FVC 2002 DB3 databases. NIST-4f contains 2000 fingerprints (500 fingerprints per class) of size $(512 \times 512)$ and one fingerprint per individual whereas DB3 contains 110 users and 8 fingerprints per user. First, the fingerprint images are given as an input to the software and minutiae templates are created. For each template, the orientation map, $\hat{\boldsymbol{\theta}}$ is estimated using minutiae triplets. Using streamlines and LIC, the ridge structure of the parent fingerprint corresponding to each template is created.

\footnotetext{
${ }^{1}$ http://www.neurotechnologija.com
} 

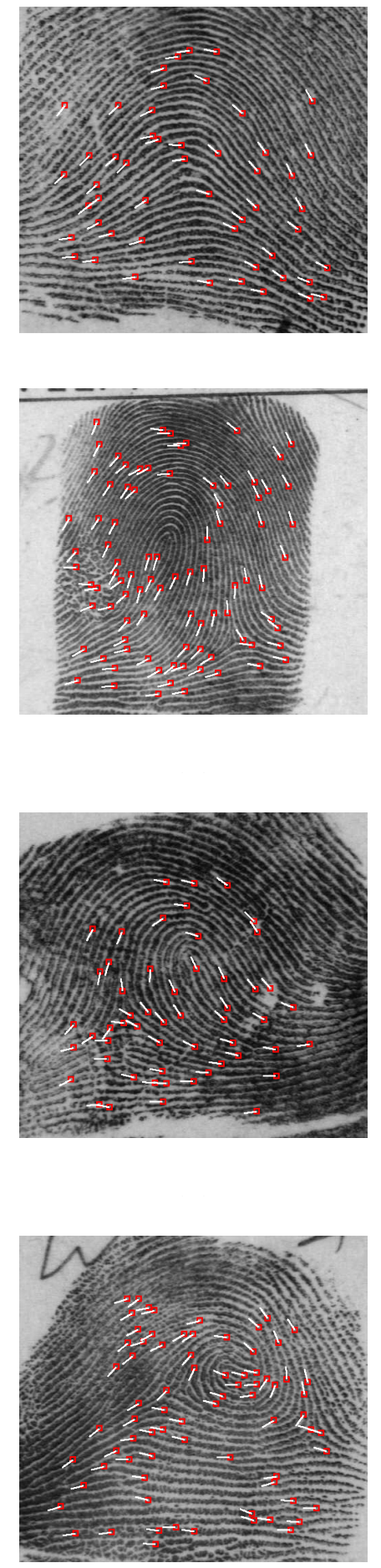

(a)

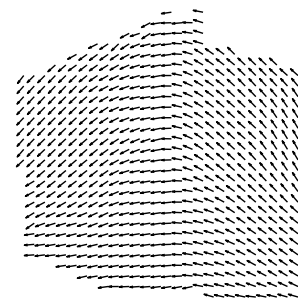

(b)

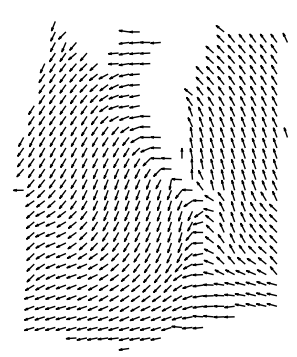

(b)

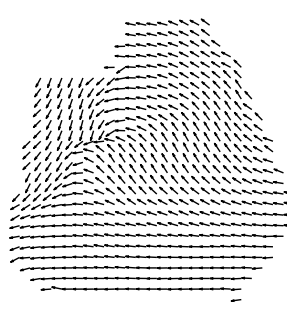

(b)

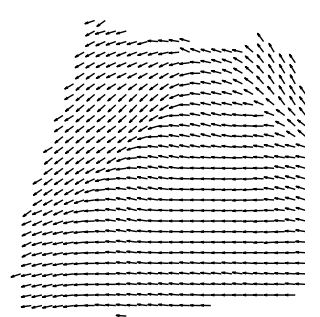

(b)
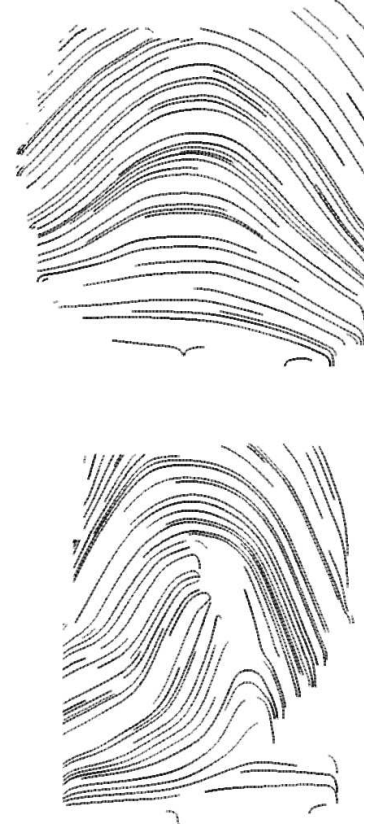

$(n)$
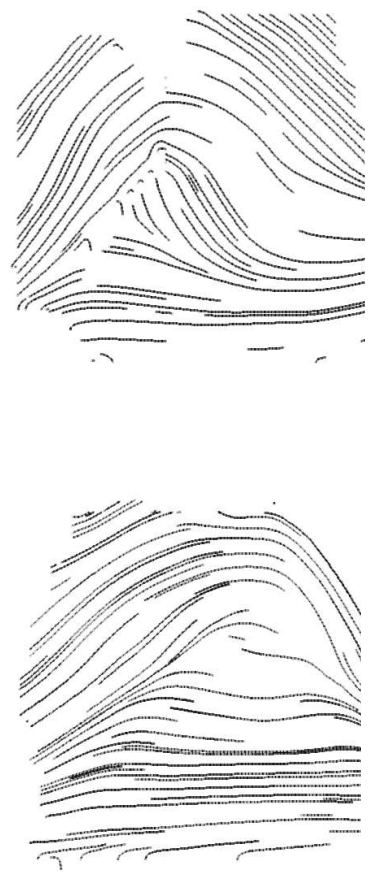

(c)

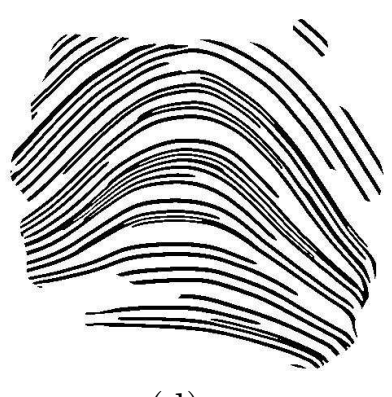

$(1)$

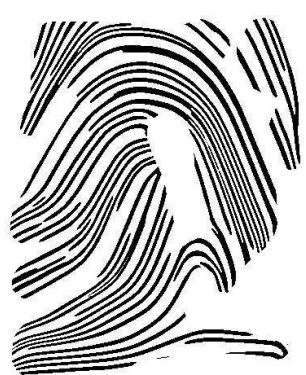

(d)
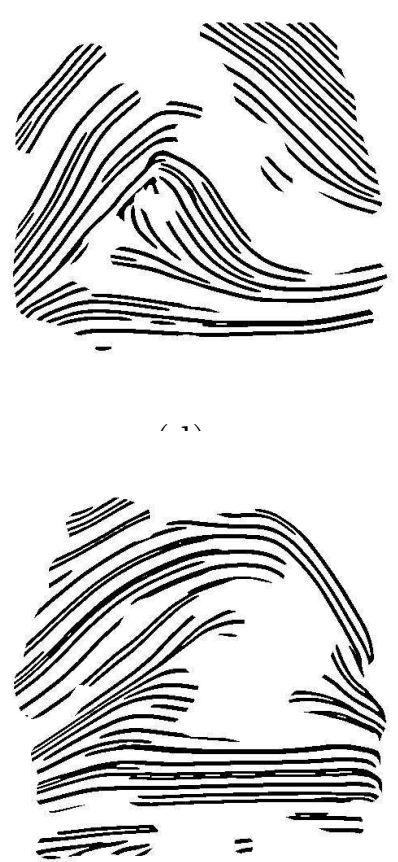

(d)

Figure 4.19: (a) Original fingerprint and its minutiae plot, (b) Estimated orientation map $\hat{\boldsymbol{\theta}}$, (c) Result of applying LIC, (d) Enhanced ridge structure using COTS. 
We considered two matching scenarios. In the first scenario, each reconstructed fingerprint was matched against every original fingerprint in the database. In the second scenario, it is assumed that the class of the parent fingerprint is known. Then, the reconstructed fingerprint is matched with all the original fingerprints of this class only. For each reconstructed fingerprint, the top matches are recorded, and the CMC (Cumulative Match Characteristics) curve is generated to summarize the identification performance. The CMC graph plots the identification rate as a function of the number of top matches (ranks) [54]. Figure 4.20 (e) shows the CMC curves. It is evident that $\mathbf{W}$ gives lower identification rates. A fingerprint of $\mathbf{W}$ class is characterized

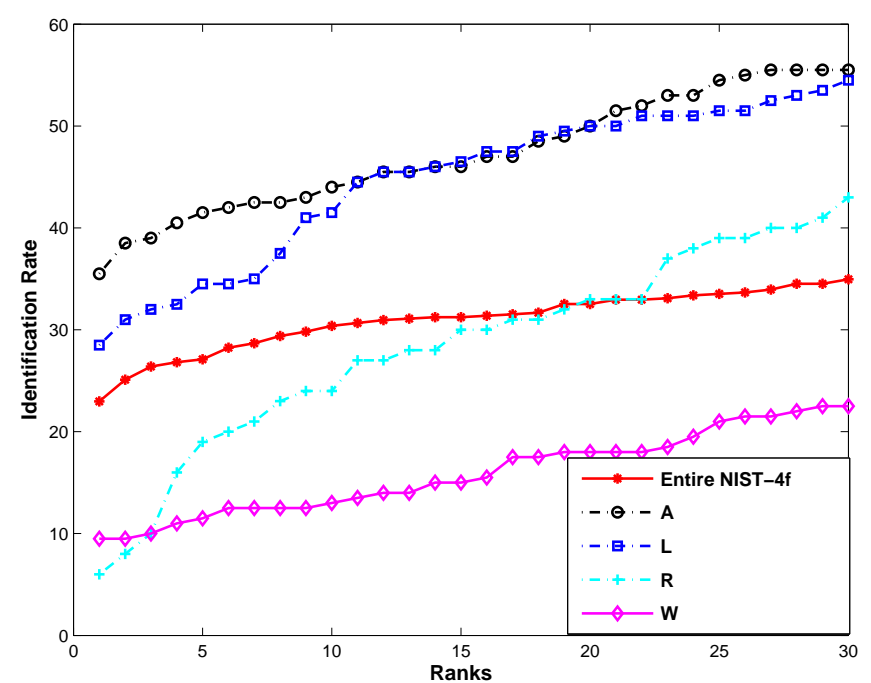

Figure 4.20: CMC curves when class of reconstructed fingerprint is (a) unknown (matched against all fingerprints of NIST-4f database), (b) known (matched against all fingerprints of the same class only).

by high ridge activity near core and delta region. The orientation estimation algorithm cannot capture the ridge orientations near the core or delta region correctly. This results in the incorrect generation of streamlines in this region. Hence, the minutiae points in this region may not match with that of the original fingerprint resulting in lower identification rate. Also, during streamline construction, we do not permit a streamline to be generated too close to any other existing streamline. As the inter-ridge distance is assumed to be absent in the minutiae template, we use a constant inter-ridge distance for the entire fingerprint. Thus, very few streamlines are generated in region with high minutiae density resulting in missing minutiae in the reconstructed fingerprint.

For the DB3 database, we performed matching using the entire database. The CMC curve for DB3 database (Figure 4.21) indicates very low identification rates. The fingerprints in this 


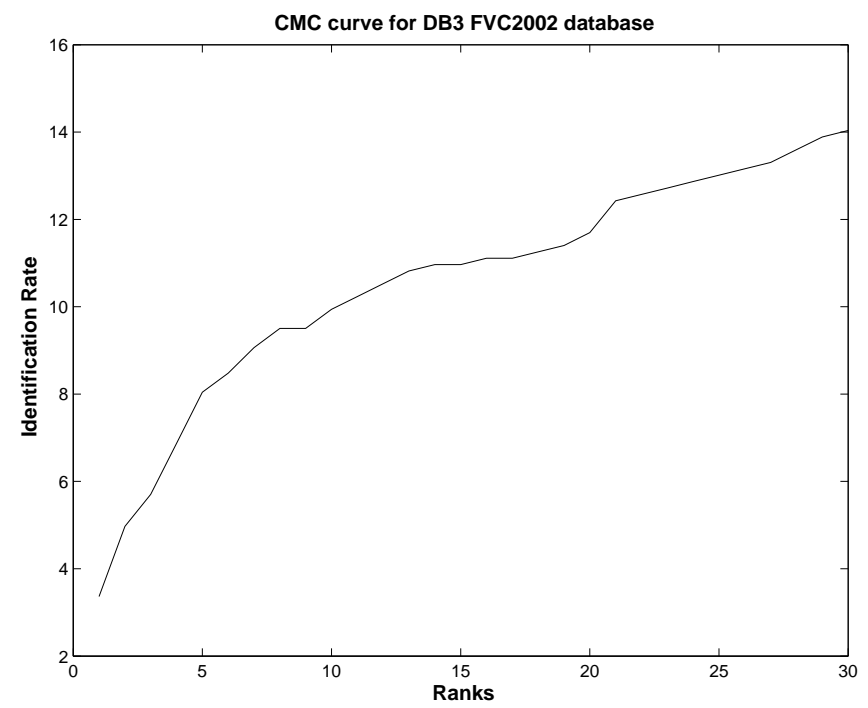

Figure 4.21: CMC curves when reconstructed fingerprint matched against all the fingerprints in FVC2002 DB3 database).

database are dab prints of size $300 \times 300$. They contain relatively small number of minutiae points. Thus the estimated orientation map $\hat{\boldsymbol{\theta}}$ has many empty cells (no estimation porssible) resulting in short streamlines as shown in Figure 4.22. When the reconstructed output is given as input to the VeriFinger software, it performs certain preprocessing techniques for enhancing the fingerprint. In some cases, in reconstructed fingerprint, if the ridge density around a minutia is low, the software removes certain portion of that ridge on which the minutia resides. This results in removing genuine minutiae and forming spurious minutiae. Thus, for reconstructed fingerprints from the DB3 database, very few minutiae are extracted resulting in low matching scores. But for the rolled prints in the NIST-4f database, due to larger sensing area, they have more number of minutiae. Thus $\hat{\boldsymbol{\theta}}$ corresponding to these fingerprints contains relatively more orientations as shown in Figure 4.23 (a). This aids in generating dense streamlines. The VeriFinger software therefore extracts more minutiae points (Figure 4.23 (c)) resulting in better match scores. 


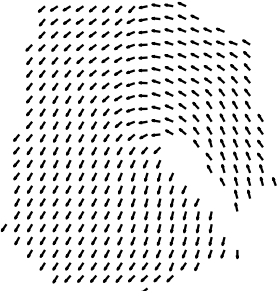

(a)

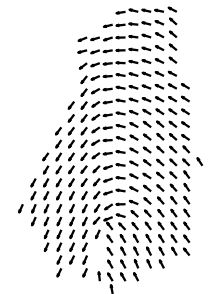

(d)

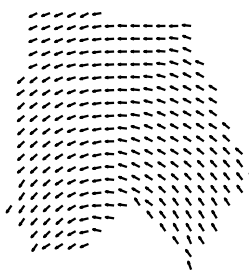

(h)

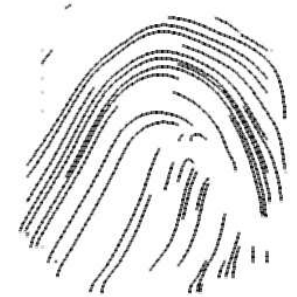

(b)

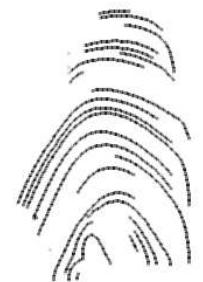

(e)

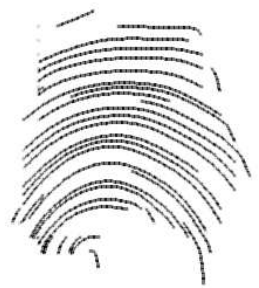

(i)

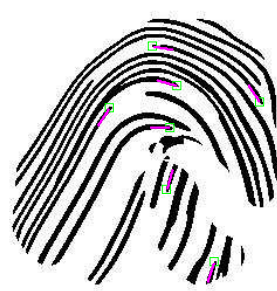

(c)

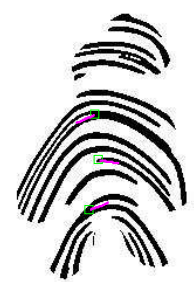

(f)

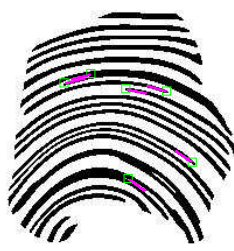

(j)

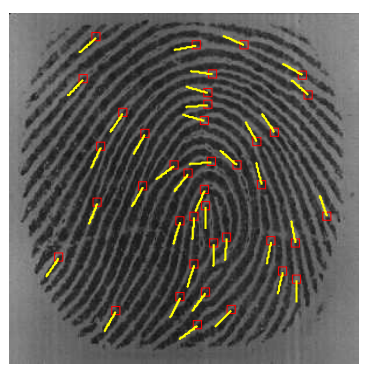

(d)

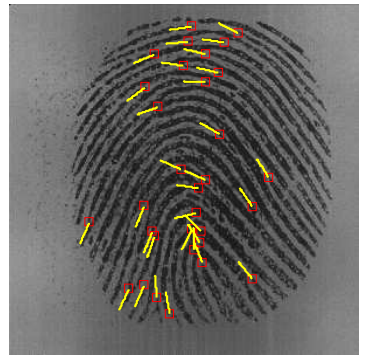

$(\mathrm{g})$

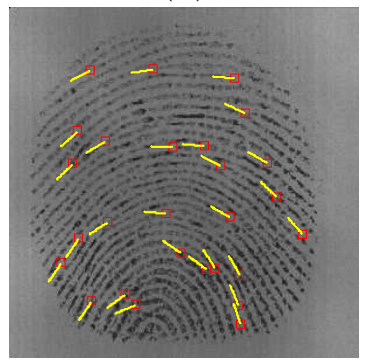

$(\mathrm{k})$

Figure 4.22: Estimated orientation map $\hat{\boldsymbol{\theta}}[(\mathrm{a}),(\mathrm{d}),(\mathrm{h})]$, Reconstructed fingerprint [(b), (e), (i)], Enhanced image by VeriFinger and its extracted minutiae $[(\mathrm{c}),(\mathrm{f}),(\mathrm{j})]$, Original fingerprint [(d), (g), (k)] for 3 fingerprints of DB3 FVC2002 database.

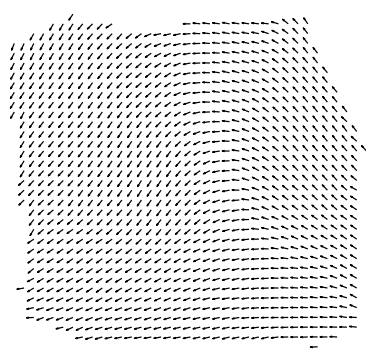

(a)

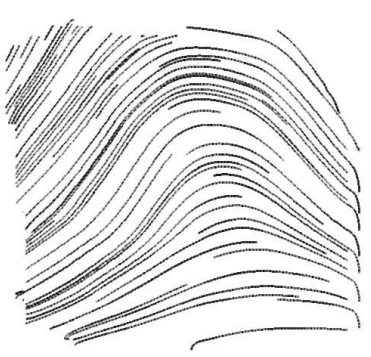

(b)

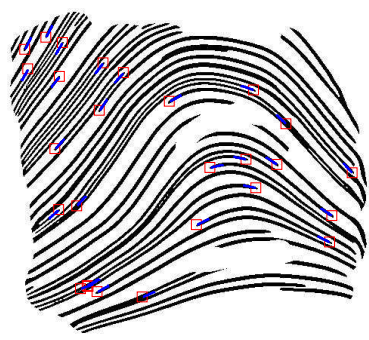

(c)

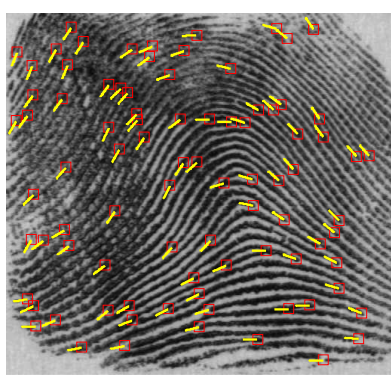

(d)

Figure 4.23: (a) Estimated orientation map $\hat{\boldsymbol{\theta}}$, (b) Reconstructed fingerprint, (c) Enhanced image by VeriFinger and its extracted minutiae, (d) Original A fingerprint of NIST 4f Database. 


\section{Chapter 5}

\section{Summary and Future work}

"First of all, it is important to remember that absolute security does not exist: given funding, willpower and the proper technology, nearly any security system can be compromised." [55].

Biometrics is a constantly growing industry and fingerprint recognition represents one of the most advantageous methods for authentication. Most fingerprint authentication systems extract and store the minutiae template of a finger after the enrollment process. It has been commonly assumed that the decrypted minutiae template does not reveal significant information about the parent fingerprint. In this thesis, we dispel this notion by demonstrating that the minutiae information may be used to derive the ridge structure of the underlying fingerprint. The proposed scheme first estimates the orientation of ridges at discrete points in a grid by considering minutiae triplets in local regions. Experiments suggest that the estimated orientations are quite consistent with the true ridge flow. This observation can be beneficial in applications like smart-cards, where the generated orientation field can be used in conjunction with the minutiae points to improve the matching performance. This estimated orientation field is then used to generate the ridge structure of the parent fingerprint by invoking streamlines and LIC. The use of streamlines permits us to control the location, type and number of minutiae in the generated ridge map. This process can also be used as an alternative technique for generating synthetic fingerprints with minutiae placed at pre-determined locations (unlike SFINGE [43] where minutiae are randomly generated). The reconstructed ridge structure is observed to be "visually" similar to that of the parent fingerprint. It may be used to spoof a fingerprint system. The exact reconstruction of the target fingerprint image is not possible as the minutiae template may not reveal important global ridge shapes. We also demonstrated that the class of a fingerprint may be inferred using 
the minutiae information alone. Thus, this research provides an insight into the individuality of fingerprints based on their minutiae content. Further, it demands the design of effective template security schemes.

We assume that the template stores minimum information about the minutiae such as $\mathrm{x}$, $\mathrm{y}$ and theta. In practice, along with this basic information, the template may also store other information such as core and delta points, minutia type, information abut neighboring minutiae, etc. [56]. Also, the fingerprints reconstructed using minutiae points can be used to masquerade not only the minutiae based authentication systems but also systems that are based on the ridge pattern of fingerprints. So proper storage and security of the template is important for the reliability and robustness of the system, and for users' trust because the acceptance of biometric systems will now depend on how securely the biometric template is stored. This research opens door for investigating methods for securing templates or making them more robust.

Encrypting the biometric template is one possible solution for masquerade attack but decrypting a template is not an impossible task. Thus, merely encrypting the template will not prevent this attack. Various methods for improving security of biometrics and stored biometric templates have been proposed in literature. Ratha et al. [57] introduced the concept of "cancellable biometrics". In this approach, the biometric signal is intentionally distorted before feature extraction. The biometric signal is distorted in the same fashion at each presentation, for enrollment and for every authentication. If one variant of the transformed biometric data is compromised, then the transform function can simply be changed. The distortion transforms are chosen to be non-invertible. So even if the transform function is known and the resulting transformed biometric data are known, the original biometrics cannot be recovered. Jain and Uludag [58 proposed a biometric watermarking technique which can increase the security of fingerprint images by hiding eigen face templates into them. Also, Linnartz and Tuyls [59] demonstrated the use of delta-contracting and epsilon-revealing functions as preprocessors for constructing helper data that is used in a way that no information about the biometric templates is leaked to imposters.

Besides all these approaches, one potential approach to prevent masquerade attack on a minutiae based fingerprint system, would be to systematically exclude certain salient minutiae from the template that are critical to image reconstruction without drastically affecting the matching performance. 
The biometric systems using identifiers other than fingerprints such as iris, hand, voice are generally template-based, i.e., they store salient features of the biometric sample in the database and use it later for matching. As Hill points out [15] the masquerade attack is applicable to many other biometrics other than fingerprints or perhaps all of them.

\section{$5.1 \quad$ Future work}

More sophisticated techniques for estimating ridge orientation from minutiae points need to be explored. Presently, the orientation scheme makes use of minutiae triplets in the local regions. The relationship between these triplets can be used to get global information about the ridge orientations. Also, techniques to estimate the orientations in the region where 'valid' triplets did not form, needs to be explored. Presently, the estimated ridge orientations in the core region can not capture important ridge properties such as circular pattern in $\mathbf{W}$ and recurving loops $(\mathbf{L}, \mathbf{R})$. Along with minutiae information, if information about core and delta is also available then a more sophisticated technique needs to be designed. The information about core and delta position can give information about class of the fingerprint which might aid in improving the orientation estimation.

Using the inferred class information from minutiae templates, the streamline construction process can be moderated since minutiae properties - such as their occurrence and density varies across classes. For example, each class can have a different seeding strategy. Verma [60] et al. discuss a seed placement strategy based on flow features in the data set. The seeds are placed in the vicinity of critical points in the flow field to capture important flow patterns. To improve the accuracy of streamline computation, we plan to use more accurate numerical integration methods like the fifth order Runga-Kutta technique.

In future, to further improve the identification performance, the fingerprints can be reconstructed using an iterative hill-climbing approach (See Figure 5.1). Here, it is assumed that the fingerprint matching software releases a match score. After reconstructing fingerprint using streamlines and LIC, the minutiae template is extracted from reconstructed fingerprint and matched against the minutiae template stored in the database using the matching software. The software releases the match score indicating similarity between the two minutiae templates. The fingerprint reconstruction process can then be modified to increase the match score. 


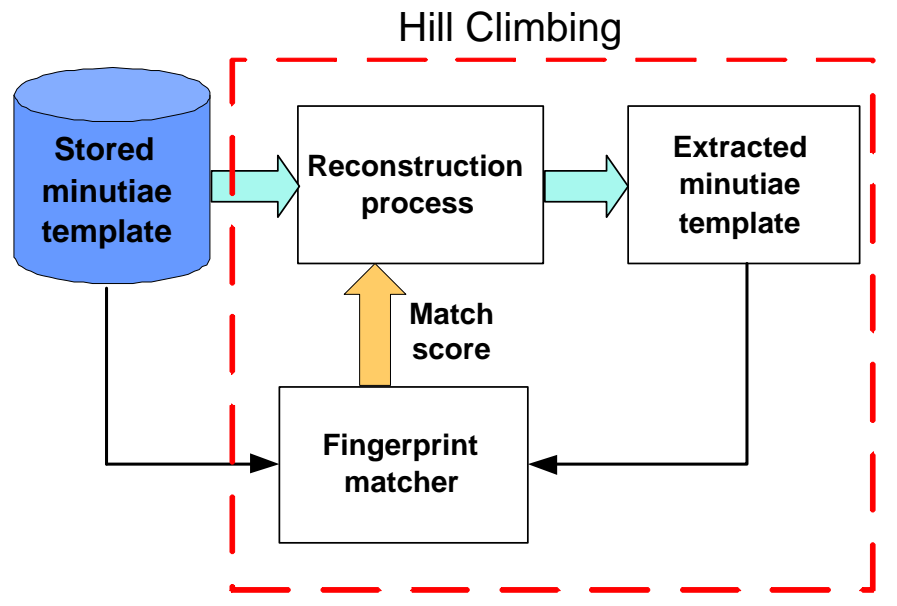

Figure 5.1: Reconstructing fingerprints using hill-climbing approach. 


\section{References}

[1] N. Ratha, J. Connell, and R. Bolle, "Analysis of minutiae matching strength," Proceedings of International Conference on Audio and Video-Based Biometric Person Authentication (3rd), vol. 24, pp. $223-228,2001$.

[2] E. Henry, Classification and Uses of Fingerprints, Routledge, London, 1900.

[3] A. K. Jain and S. Minut, "Hierarchical kernel fitting for fingerprint classification and alignment," Proc. of International Conference on Pattern Recognition (16th), vol. 2, pp. 469 473, Aug 2002.

[4] D. Maltoni, D. Maio, A. K. Jain, and S. Prabhakar, Handbook of fingerprint recognition, 1st ed. New York, Berlin Heidelberg: Springer-Verlag, 2003.

[5] J. Wayman, "Fundamentals of biometric authentication technologies."

[6] "Finger minutiae format for data interchange, ANSI/INCITS 378," American National Standard for Information Technology, [Online] Available at www.incits.org, Tech. Rep., 2004.

[7] A. Moenssens, Fingerprint Techniques. London: Chilton Book Company, 1971.

[8] D. A. Stoney, "Quantitative assessment of fingerprint individuality," Ph.D. dissertation, University of California, Davis, 1985.

[9] A. K. Jain, L. Hong, and R. Bolle, "On-line fingerprint verification," IEEE Trans on Pattern Analysis Machine Intelligence, vol. 19, no. 4, pp. 302-314, April 1997.

[10] S. Pankanti, S. Prabhakar, and A. K. Jain, "On the individuality of fingerprints," IEEE Transactions on Pattern Analysis and Machine Intelligence, vol. 24, no. 8, pp. 1010-1025, 2002.

[11] N. Ratha, J. Connell, and R. Bolle, "Biometrics break-ins and band-aids," Pattern Recognition Letters, vol. 24, pp. 2105-2113, Sep 2003.

[12] U. Uludag and A. Jain, "Attacks on biometric systems: A case study in fingerprints," Proc. SPIE-EI, Security, Seganography and Watermarking of Multimedia Contents VI, pp. 622533, Jan 2004.

[13] U. Uludag and A. K. Jain, "Fingerprint minutiae attack system," Biometrics Consortium Conference, Sep 2004.

[14] A. Adler, "Can images be regenerated from biometric templates?" Biometrics Consortium Conference, Sep 2003. 
[15] C. Hill, "Risk of masquerade arising from the storage of biometrics," Master's thesis, Australian National University, 2001.

[16] C. Soutar, "Biometric system security."

[17] "Face recongition vendor test 2002."

[18] "BioAPI speicification (version 1.1)," BioAPI Consortium, Tech. Rep., 2001.

[19] A. Adler, "Images can be generated from quantized biometric match score data," Proc. Can. Conf. Elec. Comp. Eng., Niagara Falls, May 2004.

[20] B. Sherlock and D. Monro, "A model for interpreting fingerprint topology," Pattern Recognition, vol. 26, no. 7, pp. 1047-1055, 1993.

[21] I. B. Group, "Generating images from templates," I.B.G. White Paper, Tech. Rep., 2002.

[22] N. Ratha, J. Connell, and R. Bolle, "Adaptive flow orientation-based feature extraction in fingerprint images," Pattern Recognition, vol. 28, no. 11, pp. 1657-1672, 1995.

[23] A. Rao, "A taxonomy for texture description and identification," Springer-Verlag, 1990.

[24] Z. M. Kovacs-Vajna, "A fingerprint verification system based on triangular matching and dynamic time warping," IEEE Transactions on Pattern Analysis and Machine Intelligence, vol. 22, pp. 1266 - 1276, Nov 2000.

[25] R. Germain, A. Califano, and S. Colville, "Fingerprint matching using transformation parameters clustering," IEEE Computational Science and Engineering, vol. 4, no. 4, pp. $42-$ 49, 1997.

[26] C. Kingston, "Probabilistic analysis of partial fingerprint patterns," Ph.D. dissertation, University of California, Berkeley, 1964.

[27] R. Cappelli, A. Lumini, D. Maio, and D. Maltoni, "Fingerprint classification by directional image partitioning," IEEE Transcations on Pattern Analysis and Machine Intelligence, vol. 21, pp. 402-421, 1999.

[28] G. Candela, P. Grother, C. Watson, R. Wilkinson, and C. Wilson, "PCASYS - a patternlevel classification automation system for fingerprints," NIST TR 5647, Tech. Rep., Aug 1995.

[29] A. Jain, S. Parabhakar, and L. Hong, "A multichannel approach to fingerprint classification," IEEE Transcations on Pattern Analysis and Machine Intelligence, vol. 21, pp. 348-359, 1999.

[30] L. Hong, "Automatic personal identification using fingerprints," Ph.D. dissertation, Michigan State University, 1998.

[31] M. Chong, T. Ngee, L. Jun, and R. Gay, "Geometric framework for fingerprint image classification," Pattern Recognition, vol. 30, no. 9, pp. 1475-1488, 1997.

[32] A. Senior, "A combination fingerprint classifier." IEEE Transactions on Pattern Analysis and Machine Intelligence, vol. 23, no. 10, pp. 1165-1174, 2001. 
[33] C. Wilson, G. Candela, and C. Watson, "Neural network fingerprint classification," Journal of Artificial Neural Networks, vol. 1, no. 2, pp. 203-228, 1994.

[34] K. Karu and A. Jain, "Fingerprint classification," Pattern Recognition, vol. 29, pp. 389-404, 1996.

[35] B. Cho, J. Kim, I. Bae, I. Bae, and K. Yoo, "Core-based fingerprint image classification," Proc. Int. Conf. on Pattern Recognition (15th), vol. 2, pp. 863-866, 2000.

[36] Y. Yao, P. Frasconi, and M. Pontil, "Fingerprint classification with combination of support vector machines." Proc. Int. Conf. on Audio- and Video-Based Biometric Person Authentication (3rd), pp. 253-258, 2001.

[37] A. Ross, J. Shah, and A. Jain, "Towards reconstructing fingerprints from minutiae points," Proc. of SPIE Conference on Biometric Technology for Human Identification II, pp. 68-80, 2005.

[38] T. Roxburgh, "On the evidential value of fingerprints," Sankhya: Indian Journal of Statistics, pp. 189-214, 1993.

[39] F. Galton, Finger Prints. London: McMillan, 1892.

[40] D. H. Ballard, "Generalizing the hough transform to detect arbitrary shapes," Pattern recognition, vol. 13, no. 3, pp. 111-112, 1997.

[41] C. Champod and P. Margot, "Computer assisted analysis of minutiae occurrences on fingerprints," Proc. Int'l Symp. Fingerprint Detection and Identification, p. 305, 1996.

[42] L. Hong, Y. Wan, and A. K. Jain, "Fingerprint image enhancement: Algorithm and performance evaluation," IEEE Trans on Pattern Analysis and Machine Intelligence, vol. 20, no. 8, pp. 777-789, Aug 1998.

[43] R. Cappelli, D. Maio, and D. Maltoni, "Synthetic fingerprint image generation," Proc. of 15th Int. Conf. on Advances in Pattern Recognition, vol. 3, no. 5, pp. 475-478, 2000.

[44] "Optel, Poland."

[45] S. Novikov and G. Glushchenko, "Fingerprint ridges structure generation models," Proc. SPIE, Sixth International Workshop on Digital Image Processing and Computer Graphics, vol. 3346, pp. 270-274, 1998.

[46] P. Vizcaya and L. Gerhardt, "Nonlinear orientation model for global description of fingerprints," Pattern Recognition, vol. 29, pp. 1221-1231, 1996.

[47] L. Hong, Y. Wan, and A. Jain, "Fingerprint image enhancement: Algorithm and performance evaluation," IEEE Transcations on Pattern Analysis and Machine Intelligence, vol. 20, pp. 777-789, Aug 1998.

[48] R. Cappelli, D. Maio, and D. Maltoni, "Modeling plastic distortion in fingerprint images," Proc. International Conference on Pattern Recognition 2001, pp. 369 - 376, Mar 2001.

[49] I. Jolliffe, Principal Component Analysis. Springer-Verlag, 1996. 
[50] B. Cabral and L. Leedom, "Imaging vector fields using line integral convolution," in Proceedings of the 20th annual conference on Computer graphics and interactive techniques, 1993, pp. $263-270$.

[51] D. Laidlaw, R. Kirby, C. Jackson, J. Davidson, T. Miller, M. Silva, W. Warren, and M. Tarr, "Comparing 2D vector field visualization methods: A user study," in IEEE Transactions On Visualization And Computer Graphics, vol. 11, no. 1, Jan/Feb 2005, pp. 59-70.

[52] A. Sadarjoen, T. Walsum, A. Hin, and Post, "Particle tracing algorithms for 3D curvilinear grids," In Fifth Eurographics Workshop on Visualization and Scientific Computing, 1994.

[53] B. Jobard and R. Wilfrid, "Creating evenly-spaced streamlines of arbitrary density," Visualization in Scientific Computing '9'7, Proceedings of the Eurographics Workshop in Boulognesur-Mer, 1997.

[54] H. Moon and P. Phillips, "Computational and performance aspects of pca-based facerecognition algorithms." Perception, vol. 30, pp. 303 - 321, 2001.

[55] "Fingerprint recognition based on silicon chips," Atmel Coorporation, [Online] Available at http://www.atmel.com/atmel/acrobat/wpv01.pdf, White Paper, 2001.

[56] "Minutiae interoperability exchange test 2004 (MINEX04)," NIST, [Online] Available at http://fingerprint.nist.gov/minex04/Home.html, Tech. Rep., 2004.

[57] V. Verma, D. Kao, and A. Pang, "Enhancing security and privacy in biometrics-based authentication systems," IBM Systems Journal, vol. 40, no. 3, 2001.

[58] A. Jain and U. Uludag, "Hiding biometric data," IEEE Transactions on Pattern Analysis and Machine Intelligence, vol. 25, no. 11, pp. 1494-1498, Nov 2003.

[59] J. Linnartz and P. Tuyls, "New shielding functions to enhance privacy and prevent misuse of biometric templates," Proc. of Audio- and Video-based Person Authentication, 2003.

[60] V. Verma, D. Kao, and A. Pang, "A flow-guided streamline seeding strategy," IEEE Visualization archive Proceedings of the conference on Visualization '00, pp. 163-170, 2000. 